\title{
Fast Amide Couplings in Water: Extraction, Column Chromatography, and Crystallization Not Required
}

Sudripet Sharma, ${ }^{\dagger}$ Nicklas W. Buchbinder, ${ }^{\dagger}$ Wilfried Braje, ${ }^{\ddagger}$ Sachin Handa ${ }^{\dagger, *}$

'Department of Chemistry, University of Louisville, Louisville, Kentucky 40292, United States

*AbbVie Deutschland GmbH \& Co. KG, Neuroscience Discovery Research, Ludwigshafen, Germany

*Email: sachin.handa@,louisville.edu

\section{Supporting Information}

\section{Contents}

Page

1. General experimental details

S1

2. Reaction optimizations

$\mathrm{S} 2-\mathrm{S} 5$

3. Optimized procedure for catalytic couplings

S6

4. Substrate scope

S7

5. Gram-scale reaction procedure

S8

6. Substrate scope for gram scale reactions

S9

7. Gram scale reaction of a substrate containing free -OH group

S10

8. Analytical Data

S11-S22

9. References

$\mathrm{S} 23$

10. NMR Spectra

S23-47

11. HPLC data

S48-51 


\section{GENERAL EXPERIMENTAL DETAILS}

TLC plates (UV 254 indicator, glass backed, thickness $200 \mathrm{~mm}$ ) and silica gel (standard grade, $230-400$ mesh) were purchased from Merck. Pentane and pyridine were purchased from Fisher Scientific and used as such without further purification. Pure NMR solvents were purchased from Cambridge Isotopes Laboratories. Carboxylic acids and amines were purchased from SigmaAldrich, Combi-Block and Oakwood chemicals. EDC $\cdot \mathrm{HCl}$ and other coupling reagents were purchased from Oakwood chemicals. The coupling reactions were performed in $4 \mathrm{~mL}$ close-cap microwave vials and $25 \mathrm{~mL}$ round bottom flasks. Microwave vials were purchased from VWR International and Biotage. Reaction vials were also recycled and reused. Bulk aqueous solution of surfactant PS-750-M ${ }^{1}$ was prepared in HPLC grade water and thoroughly purged with argon before use. Melting points were determined using a MEL-TEMP II melting point apparatus with samples in Kimble Kimex 51 capillaries (1.5-1.8 x $90 \mathrm{~mm}$ ). Unless otherwise mentioned, all NMR spectra were recorded at $25^{\circ} \mathrm{C}$ on Varian Unity INOVA (400 and $500 \mathrm{MHz}$ ) spectrometers. Reported chemical shifts are referenced to residual solvent peaks. HRMS spectra were obtained on a Thermo Electron MAT 95XP mass spectrometer using either electron ionization (EI) or chemical ionization (CI). Chiral HPLC data was collected using Shimadzu LG-2030C HPLC. 


\section{REACTION OPTIMIZATION}

\subsection{Coupling reagent screening}<smiles>CC(N)c1ccccc1</smiles>

1<smiles>O=C(O)c1ccccc1</smiles>

2

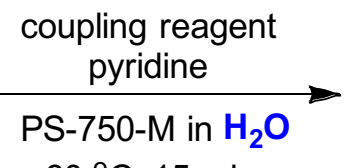

$60^{\circ} \mathrm{C}, 15 \mathrm{~min}$<smiles>CC(NC(=O)c1ccccc1)c1ccccc1</smiles>

3

\begin{tabular}{ccc}
\hline entry & coupling reagent & $\mathbf{3}(\%)$ \\
\hline $\mathbf{1}$ & EDC•HCl & $\mathbf{9 9}$ \\
2 & DCC & 15 \\
3 & HATU & 78 \\
4 & BOP & 22 \\
5 & COMU & 77
\end{tabular}

Conditions. $1(0.25 \mathrm{mmol}), 2(0.25 \mathrm{mmol})$, coupling reagent $(0.32 \mathrm{mmol})$, pyridine $(0.5 \mathrm{mmol}), 0.5 \mathrm{~mL} 3 \mathrm{wt} \%$ PS-750-M in $\mathrm{H}_{2} \mathrm{O}, 60{ }^{\circ} \mathrm{C}, 15$ min. ${ }^{a}$ Yields based on GCMS.

\subsection{Base screening}

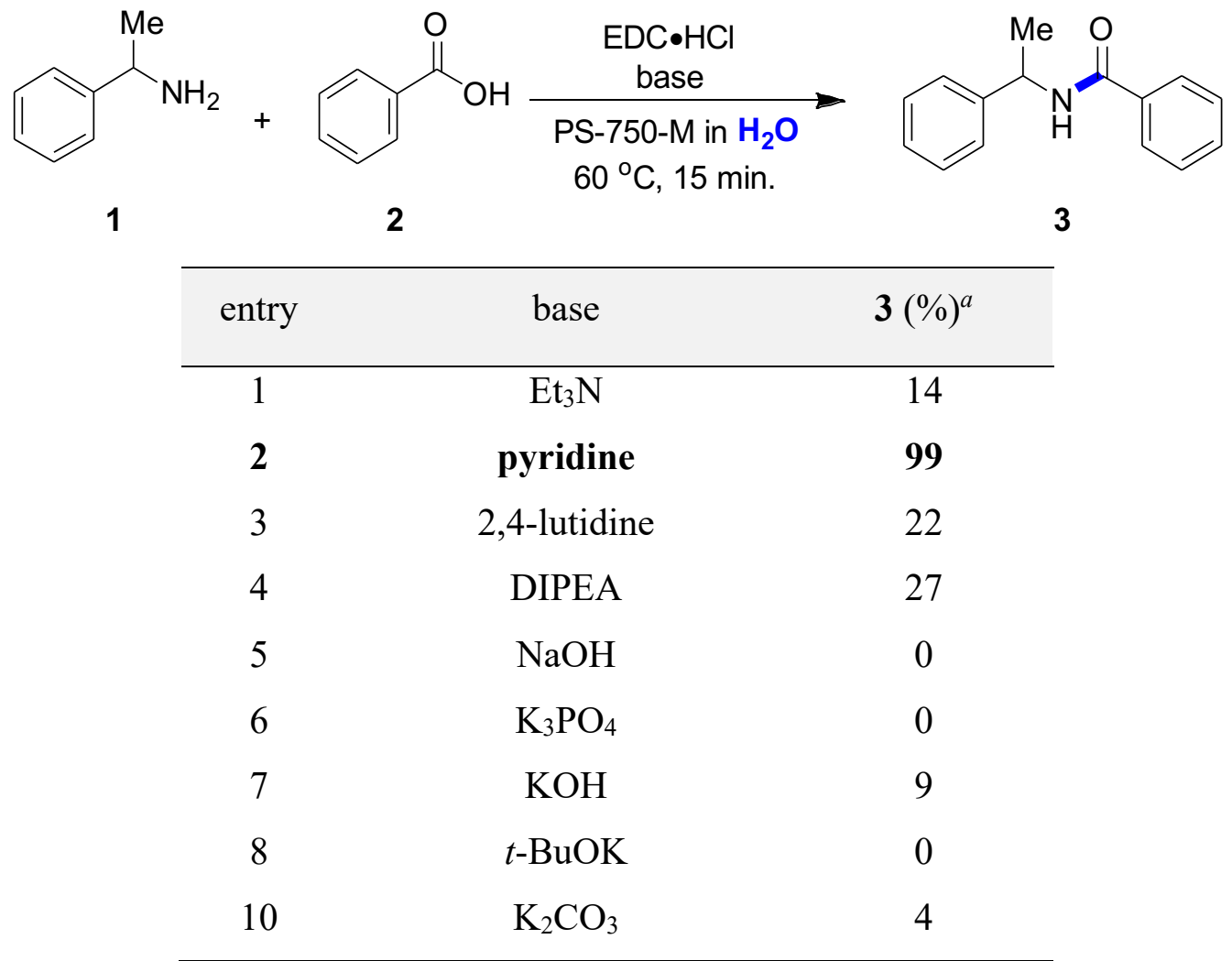


Conditions. $1(0.25 \mathrm{mmol}), 2(0.25 \mathrm{mmol}), \mathrm{EDC} \cdot \mathrm{HCl}(0.32 \mathrm{mmol})$, base $(0.5 \mathrm{mmol}), 0.5 \mathrm{~mL} 3 \mathrm{wt} \% \mathrm{PS}-750-\mathrm{M}$ in $\mathrm{H}_{2} \mathrm{O}, 60{ }^{\circ} \mathrm{C}, 15$ min. ${ }^{a}$ Yields based on GCMS.

\subsection{Effect of temperature}

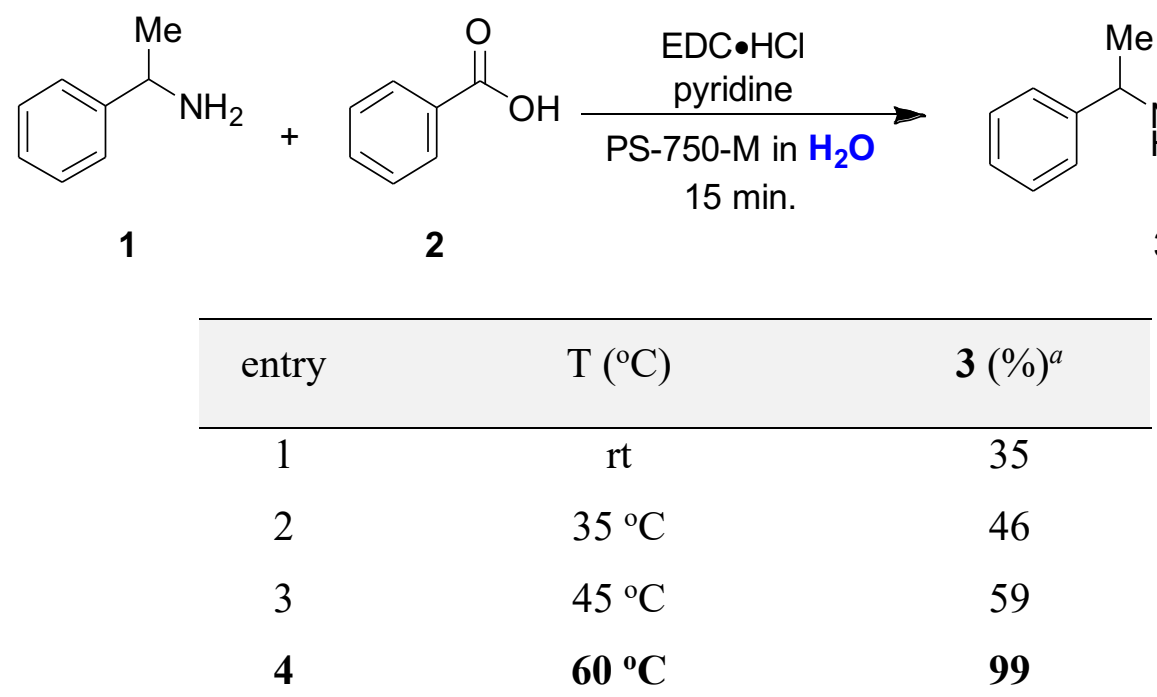

Conditions. $1(0.25 \mathrm{mmol}), 2(0.25 \mathrm{mmol}), \mathrm{EDC} \cdot \mathrm{HCl}(0.32 \mathrm{mmol})$, pyridine $(0.5 \mathrm{mmol}), 0.5 \mathrm{~mL} 3 \mathrm{wt} \% \mathrm{PS}-$ 750-M in $\mathbf{H}_{2} \mathrm{O}, 15$ min. ${ }^{a}$ Yields based on GCMS.

\subsection{Optimal equivalents of base}<smiles>CC(N)c1ccccc1</smiles>

1<smiles>O=C(O)c1ccccc1</smiles>

2

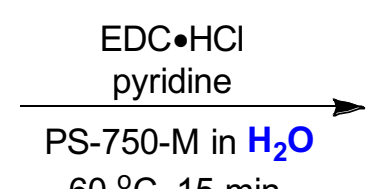
$60{ }^{\circ} \mathrm{C}, 15 \mathrm{~min}$.<smiles>CC(NC(=O)c1ccccc1)c1ccccc1</smiles>

3

\begin{tabular}{ccc}
\hline entry & pyridine (equiv.) & $\mathbf{3}(\%)^{a}$ \\
\hline 1 & 1.0 & 92 \\
2 & 1.2 & 96 \\
3 & 1.5 & 97 \\
$\mathbf{4}$ & $\mathbf{2 . 0}$ & $\mathbf{9 9}$
\end{tabular}

Conditions. $1(0.25 \mathrm{mmol}), \mathbf{2}(0.25 \mathrm{mmol}), \mathrm{EDC} \cdot \mathrm{HCl}(0.32 \mathrm{mmol})$, pyridine, $0.5 \mathrm{~mL} 3 \mathrm{wt} \% \mathrm{PS}-750-\mathrm{M}$ in $\mathrm{H}_{2} \mathrm{O}$, $60{ }^{\circ} \mathrm{C}, 15$ min. ${ }^{a}$ Yields based on GCMS. 


\subsection{Optimal global concentration}

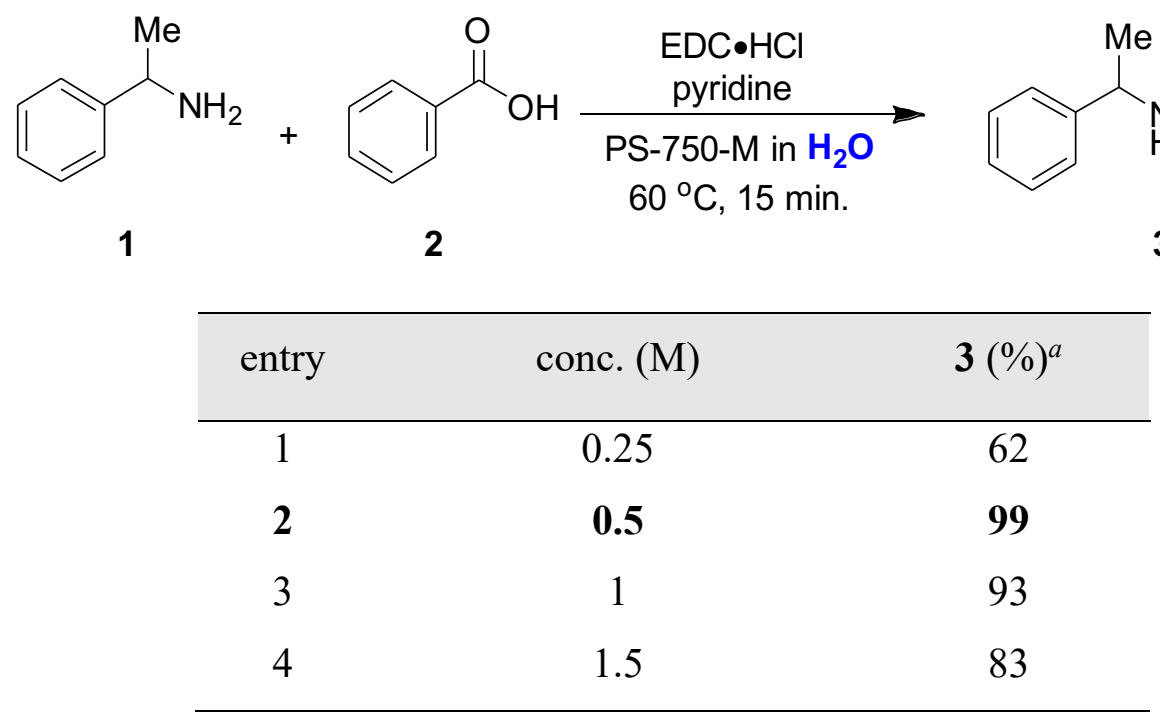

Conditions. $1(0.25 \mathrm{mmol}), 2(0.25 \mathrm{mmol}), \mathrm{EDC} \cdot \mathrm{HCl}(0.32 \mathrm{mmol})$, pyridine $(0.5 \mathrm{mmol}), 3 \mathrm{wt} \% \mathrm{PS}-750-\mathrm{M}$ in $\mathrm{H}_{2} \mathrm{O}, 60{ }^{\circ} \mathrm{C}, 15$ min. ${ }^{a}$ Yields based on GCMS.

\subsection{Optimal wt \% of PS-750-M}

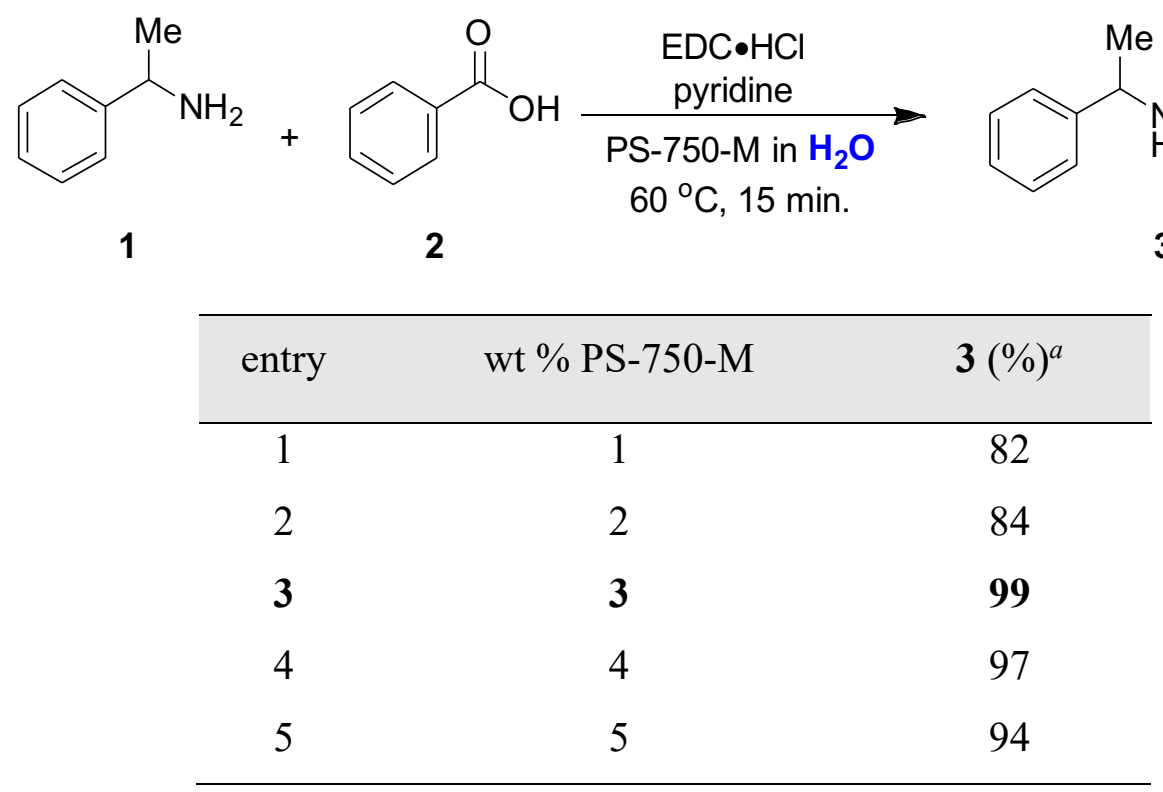

Conditions. $1(0.25 \mathrm{mmol}), 2(0.25 \mathrm{mmol}), \mathrm{EDC} \cdot \mathrm{HCl}(0.32 \mathrm{mmol})$, pyridine $(0.5 \mathrm{mmol}), 0.5 \mathrm{~mL}$ PS-750-M in $\mathrm{H}_{2} \mathrm{O}, 60{ }^{\circ} \mathrm{C}, 15$ min. ${ }^{a}$ Yields based on GCMS. 


\subsection{Optimal equivalent of $\mathrm{EDC} \cdot \mathrm{HCl}$}

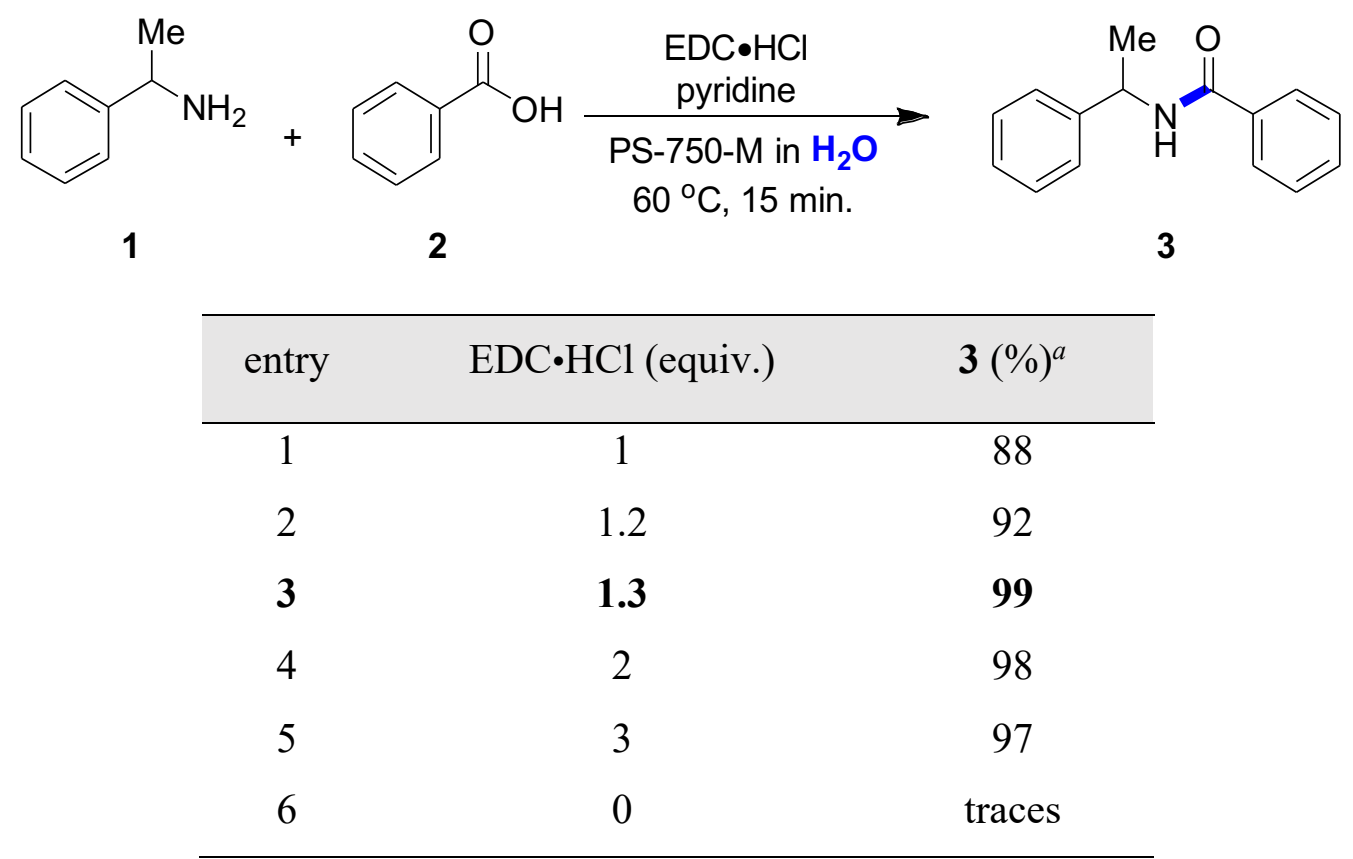

Conditions. $1(0.25 \mathrm{mmol}), 2(0.25 \mathrm{mmol}), \mathrm{EDC} \cdot \mathrm{HCl}$ (X equiv.), pyridine $(0.5 \mathrm{mmol}), 0.5 \mathrm{~mL} 3 \mathrm{wt} \% \mathrm{PS}-750$ $\mathrm{M}$ in $\mathrm{H}_{2} \mathrm{O}, 60{ }^{\circ} \mathrm{C}, 15 \mathrm{~min} .{ }^{a}$ Yields based on GCMS. 


\section{GENERAL REACTION PROCEDURE}

Carboxylic acid (0.25 mmol, 1 equiv.), amine (0.25 mmol, 1 equiv.) and EDC $\cdot \mathrm{HCl}$ (0.32 $\mathrm{mmol}$, 1.3 equiv) were taken in an oven-dried $4 \mathrm{~mL}$ reaction vial equipped with a PTFE-coated magnetic stir bar. Reaction vial was closed with a rubber septum and $0.5 \mathrm{~mL} 3 \mathrm{wt} \%$ aqueous PS-750-M was added to the reaction mixture. Septum was wrapped with PTFE tape and a parafilm. Pyridine $(0.5$ mmol, 2.0 equiv.) was subsequently added to the reaction mixture and mixture was allowed to stir at $60{ }^{\circ} \mathrm{C}$ on a pre-heated oil bath. A solid formation was observed in the reaction mixture. After reaction completion as monitored by TLC, stirring was stopped and reaction mixture was allowed to cool to rt. Solid was filtered through a Whatman filter paper. The collected solid material was washed with deionized water $(3 \times 1 \mathrm{~mL})$ and dried under reduced pressure to obtain pure product. 


\section{SUBSTRATE SCOPE}
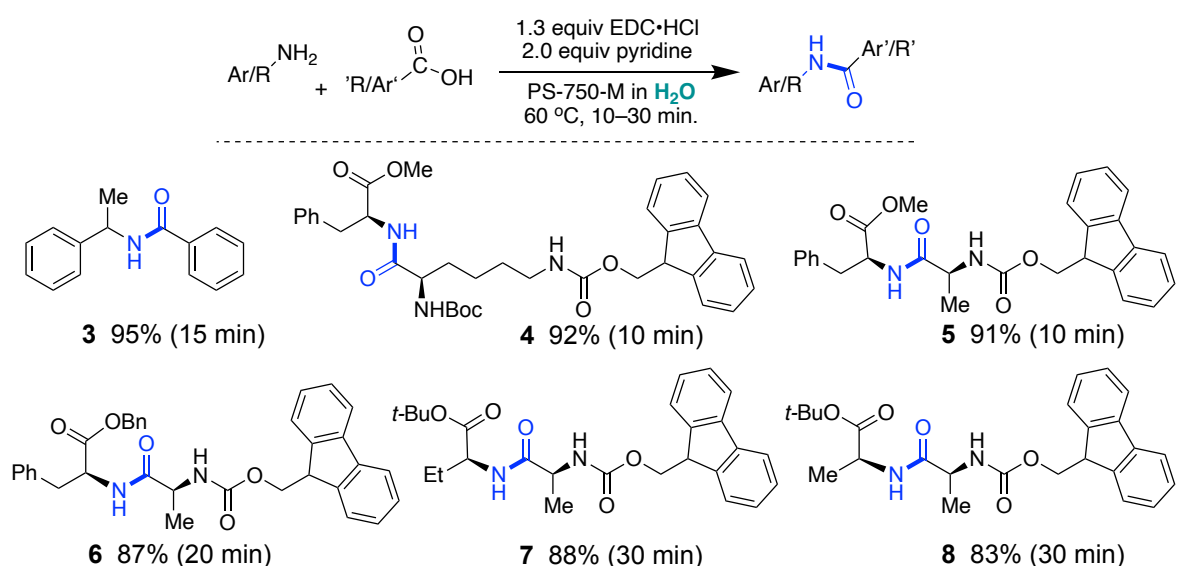

$687 \%(20 \mathrm{~min})$

7 88\% (30 min)
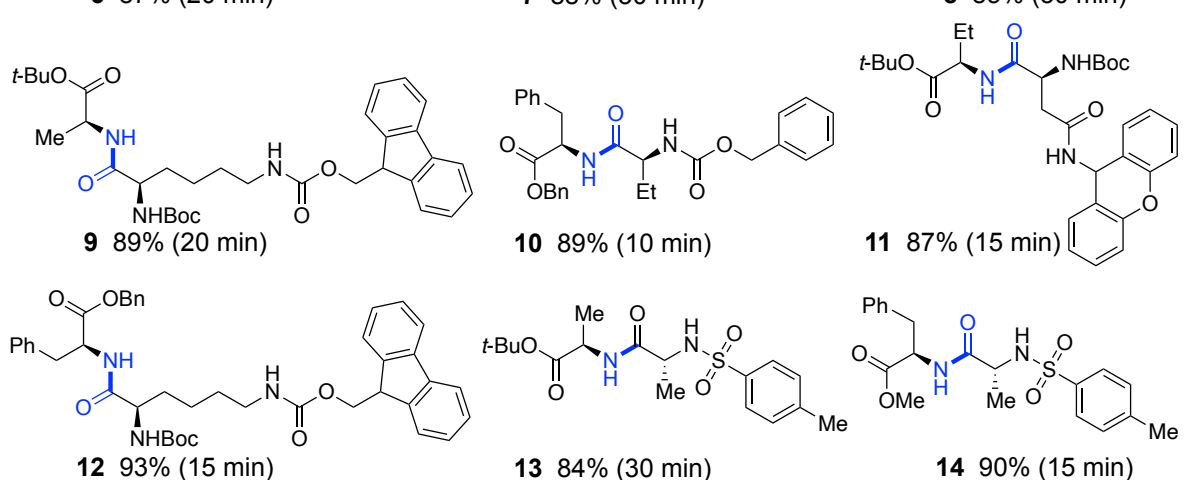

$1384 \%$ (30 min)

14 90\% (15 min)

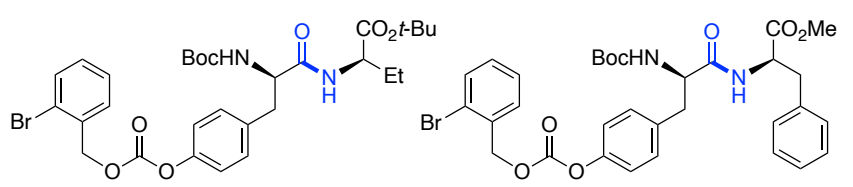

15 96\% (15 min)

16 93\% (15 min)

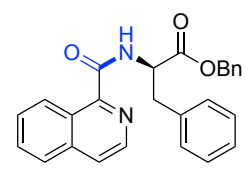

$1789 \%$ (10 min)

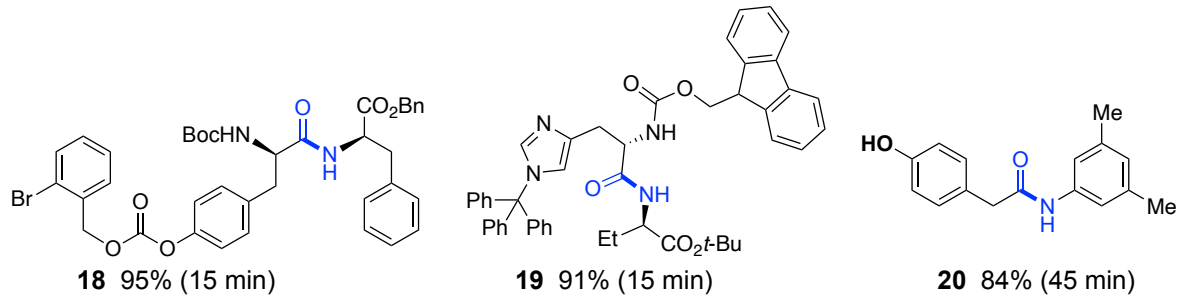<smiles>CC[C@H](NC(=O)OCc1ccccc1)C(=O)N[C@@H](Cc1ccccc1)C(=O)OC</smiles>

$2187 \%$ (10 min)

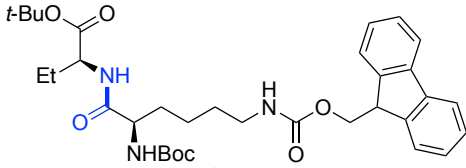

22 85\% (30 min)

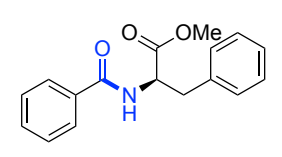

$2393 \%$ (10 min)<smiles>COC(=O)N[C@@H](CC(=O)NC1c2ccccc2Oc2ccccc21)C(=O)N[C@@H](Cc1ccccc1)C(=O)OCc1ccccc1</smiles>

24 94\% (10 $\mathrm{min})$

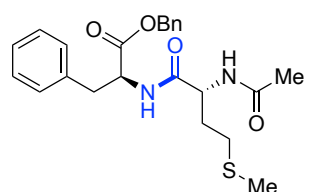

$2584 \%$ (30 min)

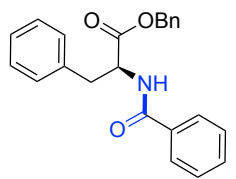

26 95\% (15 min)

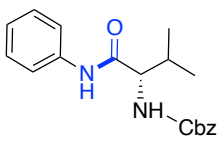

$2792 \%$ (40 min) 
Conditions: Carboxylic acid $(0.25 \mathrm{mmol})$, amine $(0.25 \mathrm{mmol}), \mathrm{EDC} \cdot \mathrm{HCl}(0.32 \mathrm{mmol})$, pyridine $(0.5 \mathrm{mmol}), 0.5 \mathrm{~mL}$ $3 \mathrm{wt} \%$ PS-750-M in $\mathrm{H}_{2} \mathrm{O}, 60^{\circ} \mathrm{C}, 10-45 \mathrm{~min}$. All yields are isolated.

\section{GENERAL PROCEDURE FOR GRAM SCALE REACTIONS}

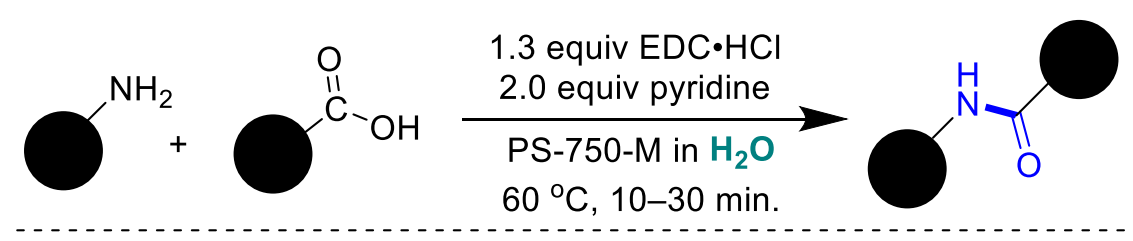

Carboxylic acid (1g, 1.0 equiv.), amine (1.0 equiv.) and $\mathrm{EDC} \cdot \mathrm{HCl}$ (1.3 equiv) were taken in an oven-dried $25 \mathrm{~mL}$ round-bottom flask equipped with a PTFE-coated magnetic stir bar. Reaction flask was closed with a rubber septum and (0.5 M) 3 wt \% aqueous PS-750-M was added to the reaction mixture. Septum was wrapped with PTFE tape and a parafilm. Pyridine (2.0 equiv.) was subsequently added to the reaction mixture and was allowed to stir at $60{ }^{\circ} \mathrm{C}$ on pre-heated oil bath for 15 - 30 minutes. A solid formation was observed in the reaction mixture. After reaction completion as monitored by TLC, stirring was stopped and reaction mixture was allowed to cool to rt. Solid was filtered through a Whatman filter paper. The collected solid material was washed with deionized water $(3 \times 3 \mathrm{~mL})$ and dried under reduced pressure to obtain pure product. The pure compound was then characterized by ${ }^{1} \mathrm{H}$ NMR and ${ }^{13} \mathrm{C}$ NMR.

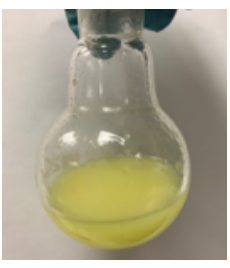

(0 min)

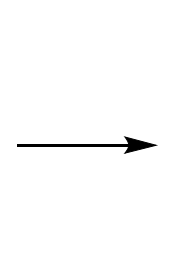

(15 min)

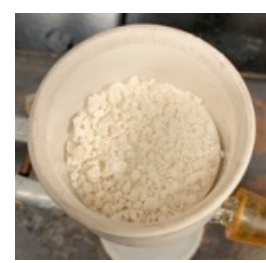

(isolated product via filteration) 


\section{SUBSTRATE SCOPE FOR GRAM SCALE REACTIONS}
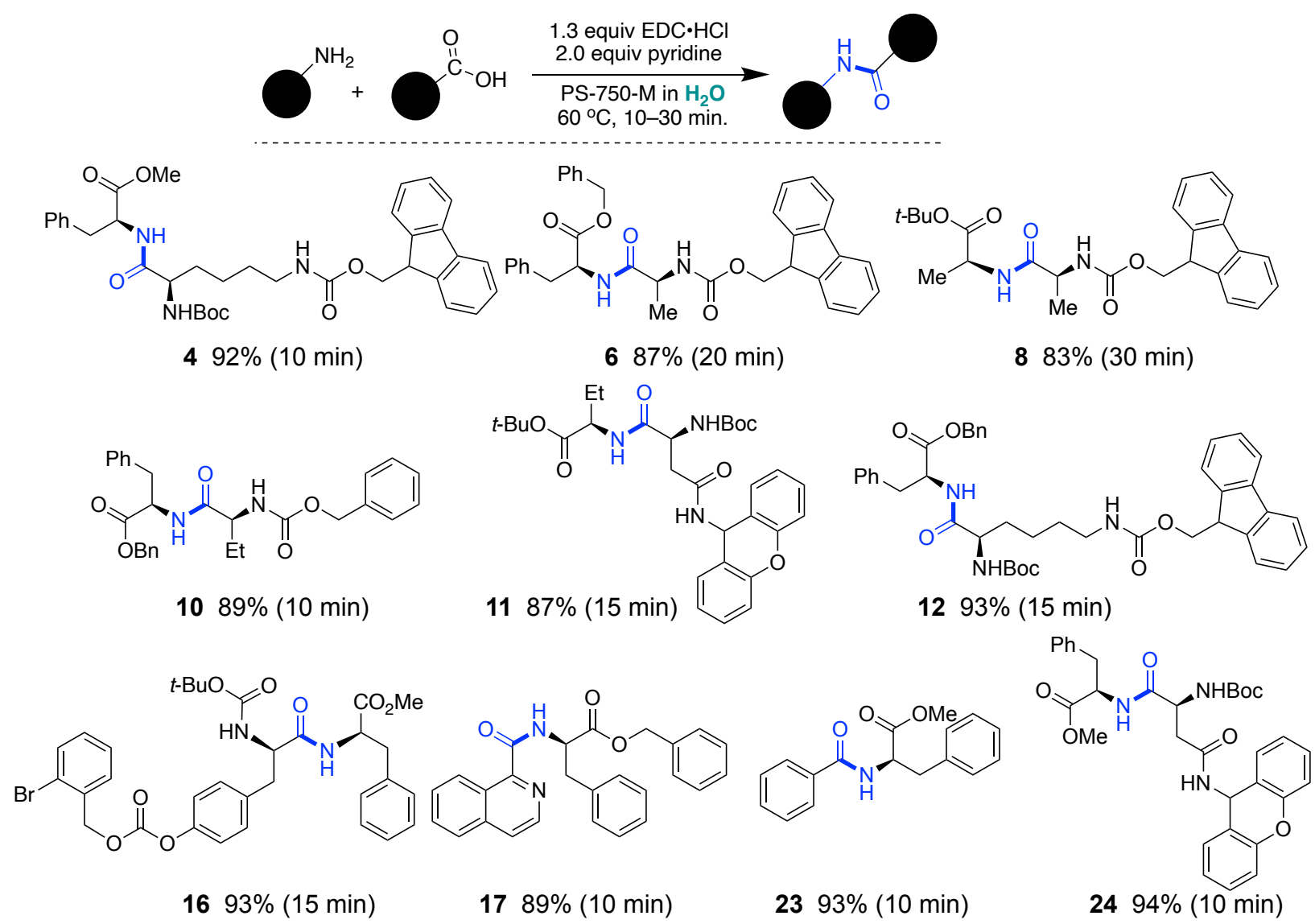

Conditions: Carboxylic acid ( $1 \mathrm{~g}, 1.0$ equiv), amine (1.0 equiv), $\mathrm{EDC} \cdot \mathrm{HCl}$ ( 1.3 equiv), pyridine ( 2 equiv), $3 \mathrm{wt} \%$ PS-750-M in $\mathrm{H}_{2} \mathrm{O}(0.5 \mathrm{M}), 60^{\circ} \mathrm{C}, 10-30 \mathrm{~min}$. All yields are isolated.

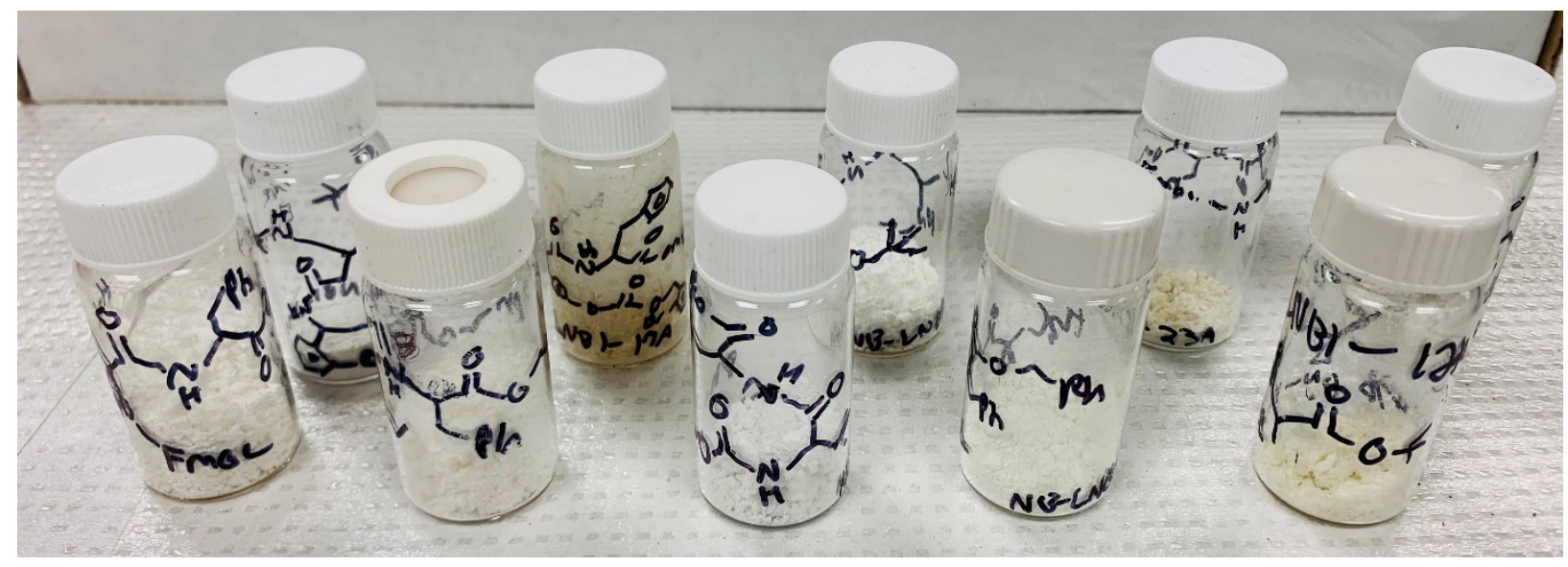




\section{GRAM SCALE REACTION OF A SUBSTRATE CONTAINING FREE -OH GROUP}<smiles>O=C(O)Cc1ccc(O)cc1</smiles>

27<smiles>Cc1cc(C)cc(N)c1</smiles>

28

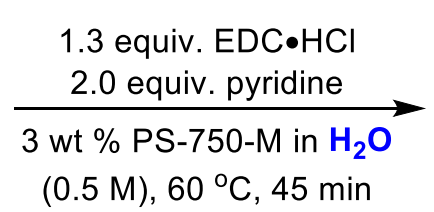

$(0.5 \mathrm{M}), 60^{\circ} \mathrm{C}, 45 \mathrm{~min}$

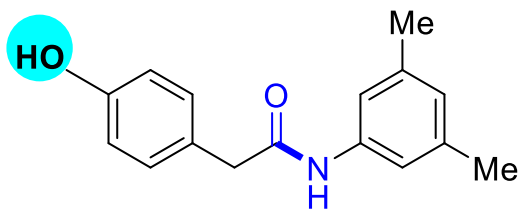

20

4-Hydroxyphenylacetic acid (1 g, 6.57 mmol, 1 equiv.), 3,5-dimethylaniline (796.5 mg, 6.57 mmol, 1 equiv.), and $\mathrm{EDC} \cdot \mathrm{HCl}(1.64 \mathrm{~g}, 8.54 \mathrm{mmol}, 1.3$ equiv) were taken in an oven-dried $25 \mathrm{~mL}$ round-bottom flask equipped with a PTFE-coated magnetic stir bar. Reaction flask was closed with a rubber septum and $13 \mathrm{~mL}$ of $3 \mathrm{wt} \%$ aqueous PS-750-M was added to the reaction mixture. Septum was wrapped with PTFE tape and a parafilm. Pyridine (1 mL, 13.14 mmol, 2 equiv.) was subsequently added to the reaction mixture and mixture was allowed to stir at $60{ }^{\circ} \mathrm{C}$ on pre-heated oil bath for 45 minutes. A solid formation was observed in the reaction mixture. After reaction completion as monitored by TLC (ethyl acetate/hexane, 2:3), stirring was stopped and reaction mixture was cooled to rt. Solid was filtered through a Whatman filter paper and washed with deionized water $(3 \times 3 \mathrm{~mL})$. Solid collected was dried under reduced pressure to obtain $N-(3,5-$ dimethylphenyl)-2-(4-hydroxyphenyl)acetamide (20) as final product. ${ }^{3}$ The pure compound was then characterized by ${ }^{1} \mathrm{H}$ and ${ }^{13} \mathrm{C}$ NMR $(1.42 \mathrm{~g}, 86 \%)$.

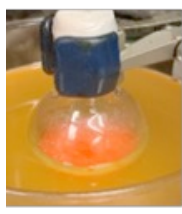

(0 $\min )$

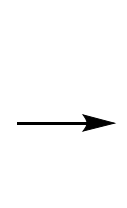

(15 min)

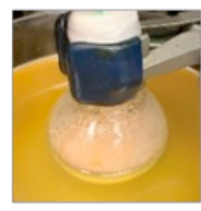

(45 $\min )$

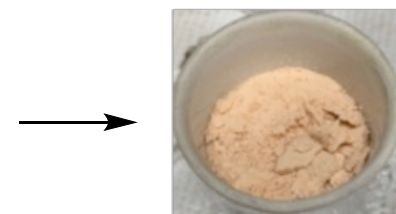

(isolated product via filteration) 


\section{ANALYTICAL DATA}

$N$-(1-phenylethyl)benzamide ${ }^{2}$ (3)<smiles>CC(NC(=O)c1ccccc1)c1ccccc1</smiles>

White solid, yield $54 \mathrm{mg}(95 \%), \mathrm{mp}=122-123{ }^{\circ} \mathrm{C}$ (lit. 120-122 $\left.{ }^{\circ} \mathrm{C}\right)^{2}, \mathrm{R}_{f} 0.4$ (7:3, hexanes/ethyl acetate). ${ }^{1} \mathbf{H}$ NMR (400 MHz, $\left.\mathbf{C D C l}_{3}\right) \delta$ 7.88-7.70 (m, 2H), 7.56-7.47 (m, 1H), 7.46-7.33 (m, 6H), 7.32-7.26 (m, 1H), $6.31(\mathrm{~d}, J=6.0 \mathrm{~Hz}, 1 \mathrm{H}), 5.45-5.28(\mathrm{~m}, 1 \mathrm{H}), 1.62(\mathrm{~d}, J=6.9 \mathrm{~Hz}, 3 \mathrm{H})$.

Methyl $\quad N$-(((9H-fluoren-9-yl)methoxy)carbonyl)- $N^{2}$-(tert-butoxycarbonyl)- $L$-lysyl- $L$ phenylalaninate (4)

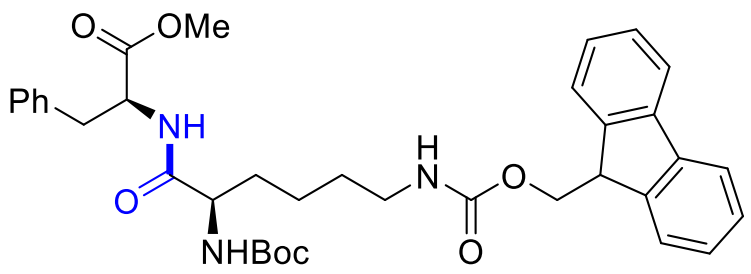

White solid, yield $148 \mathrm{mg}(92 \%), \mathrm{mp}=133-134{ }^{\circ} \mathrm{C}, \mathrm{R}_{f} 0.3\left(9: 1, \mathrm{CH}_{2} \mathrm{Cl}_{2} / \mathrm{CH}_{3} \mathrm{OH}\right) .{ }^{1} \mathrm{H}$ NMR (400 MHz, $\left.\mathbf{C D C l}_{3}\right) \delta 7.78(\mathrm{~d}, J=7.5 \mathrm{~Hz}, 2 \mathrm{H}), 7.60(\mathrm{~d}, J=7.4 \mathrm{~Hz}, 2 \mathrm{H}), 7.40(\mathrm{~m}, 2 \mathrm{H}), 7.35-7.23(\mathrm{~m}$, $5 \mathrm{H}), 7.12$ (d, $J=7.0 \mathrm{~Hz}, 2 \mathrm{H}), 6.46$ (d, $J=8.0 \mathrm{~Hz}, 1 \mathrm{H}), 5.05(\mathrm{~s}, 1 \mathrm{H}), 4.94(\mathrm{~s}, 1 \mathrm{H}), 4.86(\mathrm{dd}, J=$ 13.8, 6.2 Hz, 1H), $4.40(\mathrm{t}, J=6.6 \mathrm{~Hz}, 2 \mathrm{H}), 4.22(\mathrm{t}, J=7.1 \mathrm{~Hz}, 1 \mathrm{H}), 4.06(\mathrm{~s}, 1 \mathrm{H}), 3.70(\mathrm{~s}, 3 \mathrm{H}), 3.26-$ 3.10 (m, 4H), 1.78-1.70 (m, 2H), 1.59-1.32 (m, 13H). ${ }^{13} \mathbf{C}$ NMR (100 MHz, CDCl $) \delta 171.9$, $171.8,156.7,155.7,144.1,141.4,135.8,129.3,128.7,127.8,127.3,127.1,125.2,120.1,70.7$, 66.7, 54.4, 53.2, 52.5, 47.4, 40.5, 38.0, 32.0, 29.5, 28.4, 22.5. IR $v\left(\mathrm{~cm}^{-1}\right) 3323(\mathrm{~m}), 2939(\mathrm{~m})$, 1706 (s), 1524 (s). HRMS (ESI) m/z: [M + Na] $]^{+}$Calcd for $\mathrm{C}_{36} \mathrm{H}_{43} \mathrm{~N}_{3} \mathrm{O}_{7} \mathrm{Na}$ 652.2993; found 652.2995 .

Methyl (((9H-fluoren-9-yl)methoxy)carbonyl)- $L$-alanyl- $L$-phenylalaninate (5)<smiles>COC(=O)[C@H](Cc1ccccc1)NC(=O)[C@H](C)NC(=O)OCC1c2ccccc2-c2ccccc21</smiles> 
White solid, yield $108 \mathrm{mg}(91 \%), \mathrm{mp}=168-170{ }^{\circ} \mathrm{C}, \mathrm{R}_{f} 0.3\left(9.5: 0.5, \mathrm{CH}_{2} \mathrm{Cl}_{2} / \mathrm{CH}_{3} \mathrm{OH}\right) .{ }^{1} \mathbf{H}$ NMR $\left(400 \mathrm{MHz}, \mathbf{C D C l}_{3}\right) \delta 7.38(\mathrm{~d}, J=7.5 \mathrm{~Hz}, 2 \mathrm{H}), 7.20(\mathrm{~d}, J=7.0 \mathrm{~Hz}, 2 \mathrm{H}), 7.02(\mathrm{t}, J=7.4 \mathrm{~Hz}, 2 \mathrm{H})$, $6.94(\mathrm{t}, J=7.4 \mathrm{~Hz}, 2 \mathrm{H}), 6.88-6.75(\mathrm{~m}, 3 \mathrm{H}), 6.68(\mathrm{~d}, J=7.0 \mathrm{~Hz}, 2 \mathrm{H}), 6.00(\mathrm{~s}, 1 \mathrm{H}), 4.90(\mathrm{~s}, 1 \mathrm{H})$, $4.47(\mathrm{q}, J=12 \mathrm{~Hz}, 1 \mathrm{H}), 3.98(\mathrm{~m}, 2 \mathrm{H}), 3.82(\mathrm{~m}, 2 \mathrm{H}), 3.33(\mathrm{~s}, 3 \mathrm{H}), 2.73(\mathrm{~m}, 2 \mathrm{H}), 0.97(\mathrm{~d}, J=6.1$ $\mathrm{Hz}, 3 \mathrm{H}) .{ }^{13} \mathbf{C}$ NMR (100 MHz, $\left.\mathbf{C D C l}_{3}\right) \delta 171.9,171.8,156,143.9,141.4,135.7,129.3,128.7$, 127.9, 127.3, 125.2, 120.1, 70.7, 67.3, 53.3, 52.5, 50.5, 47.2, 37.9, 18.7. IR v (cm-1) $3318(\mathrm{~s}), 2944$ (w), 1622 (s), 1522 (w). HRMS (ESI) m/z: [M + H] Calcd for $\mathrm{C}_{28} \mathrm{H}_{29} \mathrm{~N}_{2} \mathrm{O}_{5}$ 473.2076; found 473.2070.

\section{Benzyl (((9H-fluoren-9-yl)methoxy)carbonyl)- $L$-alanyl- $L$-phenylalaninate (6)}

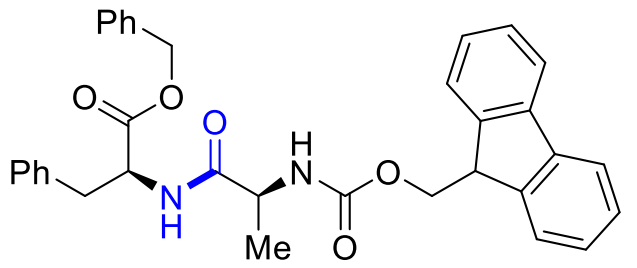

White solid, yield $119.5 \mathrm{mg}(87 \%), \mathrm{mp}=174-175^{\circ} \mathrm{C}, \mathrm{R}_{f} 0.3\left(9: 1, \mathrm{CH}_{2} \mathrm{Cl}_{2} / \mathrm{CH}_{3} \mathrm{OH}\right) .{ }^{1} \mathbf{H}$ NMR (400 MHz, $\left.\mathbf{C D C l}_{3}\right) \delta 7.77(\mathrm{~d}, J=7.5 \mathrm{~Hz}, 2 \mathrm{H}), 7.59(\mathrm{~d}, J=6.9 \mathrm{~Hz}, 2 \mathrm{H}), 7.47-7.29(\mathrm{~m}, 9 \mathrm{H}), 7.16(\mathrm{~d}, J=$ $2.6 \mathrm{~Hz}, 3 \mathrm{H}), 6.98(\mathrm{~s}, 2 \mathrm{H}), 6.35$ (d, J=8.5 Hz, 1H), $5.27(\mathrm{~s}, 1 \mathrm{H}), 5.22-5.06(\mathrm{~m}, 2 \mathrm{H}), 4.89(\mathrm{~m}, 1 \mathrm{H})$, 4.46-4.28 (m, 2H), $4.21(\mathrm{~m}, 2 \mathrm{H}), 3.12(\mathrm{~m}, 2 \mathrm{H}), 1.34(\mathrm{~d}, J=6.6 \mathrm{~Hz}, 3 \mathrm{H}) .{ }^{13} \mathbf{C}$ NMR (100 MHz, $\left.\mathbf{C D C l}_{3}\right) \delta 171.9,171.1,156,143.9,141.4,135.5,135.1,129.4,128.8,127.9,127.2,125.2,120.1$, 67.5, 67.3, 53.3, 50.5, 47.2, 37.9, 18.7. IR v ( $\left.\mathrm{cm}^{-1}\right) 3323$ (s), 2976 (w), 1634 (s), 1541 (w). HRMS (ESI) $\mathrm{m} / \mathrm{z}:[\mathrm{M}+\mathrm{Na}]^{+}$Calcd for $\mathrm{C}_{34} \mathrm{H}_{32} \mathrm{~N}_{2} \mathrm{O}_{5} \mathrm{Na} 571.2209$; found 571.2203.

\section{Tert-butyl (S)-2-((S)-2-((((9H-fluoren-9-yl)methoxy)carbonyl)amino)propanamido)} butanoate (7)

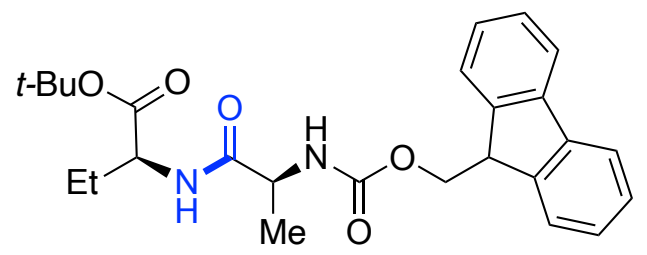

White solid, yield $103 \mathrm{mg}(88 \%), \mathrm{mp}=187-189^{\circ} \mathrm{C}, \mathrm{R}_{f} 0.3$ (9:1, $\left.\mathrm{CH}_{2} \mathrm{Cl}_{2} / \mathrm{CH}_{3} \mathrm{OH}\right) .{ }^{1} \mathbf{H}$ NMR (400 MHz, $\left.\mathbf{C D C l}_{3}\right) \delta 7.76(\mathrm{~d}, J=7.5 \mathrm{~Hz}, 2 \mathrm{H}), 7.59(\mathrm{~d}, J=7.4 \mathrm{~Hz}, 2 \mathrm{H}), 7.40(\mathrm{t}, J=7.5 \mathrm{~Hz}, 2 \mathrm{H}), 7.31$ $(\mathrm{t}, J=7.4 \mathrm{~Hz}, 2 \mathrm{H}), 6.51(\mathrm{~d}, J=6.3 \mathrm{~Hz}, 1 \mathrm{H}), 5.42$ (d, $J=7.3 \mathrm{~Hz}, 1 \mathrm{H}), 4.54-4.37(\mathrm{~m}, 3 \mathrm{H}), 4.34-4.27$ 
$(\mathrm{m}, 1 \mathrm{H}), 4.23(\mathrm{t}, J=7.1 \mathrm{~Hz}, 1 \mathrm{H}), 1.97-1.82(\mathrm{~m}, 1 \mathrm{H}), 1.78-1.64(\mathrm{~m}, 1 \mathrm{H}), 1.46(\mathrm{~s}, 9 \mathrm{H}), 1.42(\mathrm{~d}, J=$ $6.7 \mathrm{~Hz}, 3 \mathrm{H}), 0.88$ (t, $J=7.2 \mathrm{~Hz}, 3 \mathrm{H}) .{ }^{13} \mathbf{C}$ NMR (100 MHz, CDCl $\left.\mathbf{3}\right) \delta 171.9,171.3,156,143.9$, $141.4,127.9,127.2,125.2,120.1,82.4,67.3,53.8,50.7,47.3,28.1,25.7,19.2,9.2$. IR $v\left(\mathrm{~cm}^{-1}\right)$ 3313 (s), 2987 (w), 1643 (s), 1532 (w). HRMS (ESI) m/z: [M + Na] Calcd for $\mathrm{C}_{26} \mathrm{H}_{32} \mathrm{~N}_{2} \mathrm{O}_{5} \mathrm{Na}$ 475.2209; found 475.2203.

Tert-butyl (((9H-fluoren-9-yl)methoxy)carbonyl)- $L$-alanyl- $L$-alaninate (8)

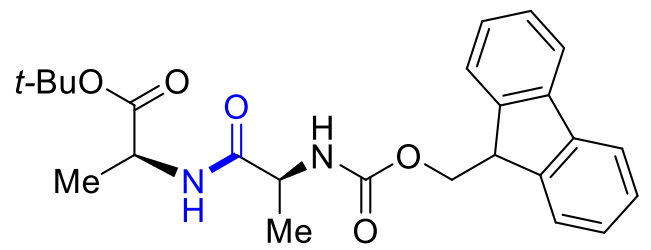

White solid, yield $94 \mathrm{mg}(83 \%), \mathrm{mp}=162-164{ }^{\circ} \mathrm{C}, \mathrm{R}_{f} 0.3\left(9: 1, \mathrm{CH}_{2} \mathrm{Cl}_{2} / \mathrm{CH}_{3} \mathrm{OH}\right) .{ }^{1} \mathbf{H}$ NMR (400 MHz, $\left.\mathbf{C D C l}_{3}\right) \delta 7.76(\mathrm{~d}, J=7.6 \mathrm{~Hz}, 2 \mathrm{H}), 7.59(\mathrm{~d}, J=7.4 \mathrm{~Hz}, 2 \mathrm{H}), 7.40(\mathrm{t}, J=7.4 \mathrm{~Hz}, 2 \mathrm{H}), 7.31$ $(\mathrm{t}, J=7.4 \mathrm{~Hz}, 2 \mathrm{H}), 6.40(\mathrm{~d}, J=6.1 \mathrm{~Hz}, 1 \mathrm{H}), 5.41(\mathrm{~d}, J=8.1 \mathrm{~Hz}, 1 \mathrm{H}), 4.69-4.33(\mathrm{~m}, 3 \mathrm{H}), 4.22(\mathrm{t}$, $J=6.9 \mathrm{~Hz}, 2 \mathrm{H}), 1.46(\mathrm{~s}, 9 \mathrm{H}), 1.41(\mathrm{~d}, J=6.7 \mathrm{~Hz}, 3 \mathrm{H}), 1.38(\mathrm{~d}, J=7.1 \mathrm{~Hz}, 3 \mathrm{H}) .{ }^{13} \mathbf{C}$ NMR (100 MHz, $\left.\mathbf{C D C l}_{3}\right) \delta 172,171.6,169,144,141.5,127.9,127.2,125.2,120.1,82.4,67.2,50.6,48.9$, 47.3, 28.1, 19.1, 18.7. IR v (cm-1) 3294 (m), 2767 (w), 1753 (s), 1636 (s). HRMS (ESI) m/z: [M + $\mathrm{Na}]^{+}$Calcd for $\mathrm{C}_{25} \mathrm{H}_{30} \mathrm{~N}_{2} \mathrm{O}_{5} \mathrm{Na}$ 461.2052; found 461.2047.

Tert-butyl $\quad N^{6}$-(((9H-fluoren-9-yl)methoxy)carbonyl)- $N^{2}$-(tert-butoxycarbonyl)- $L$-lysyl- $L$ alaninate (9)

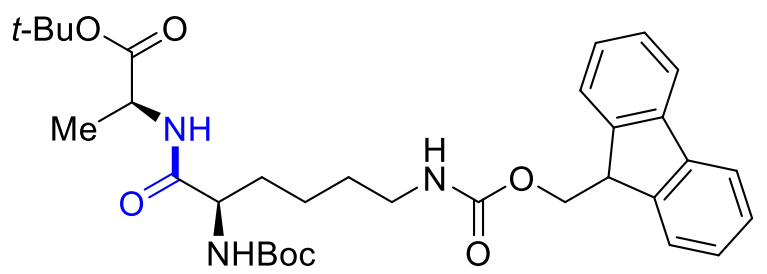

White solid, yield $136 \mathrm{mg}(89 \%), \mathrm{mp}=120-121{ }^{\circ} \mathrm{C}, \mathrm{R}_{f} 0.3\left(9: 1, \mathrm{CH}_{2} \mathrm{Cl}_{2} / \mathrm{CH}_{3} \mathrm{OH}\right) .{ }^{1} \mathbf{H}$ NMR (400 MHz, $\left.\mathbf{C D C l}_{3}\right) \delta 7.76(\mathrm{~d}, J=7.5 \mathrm{~Hz}, 2 \mathrm{H}), 7.59(\mathrm{~d}, J=7.4 \mathrm{~Hz}, 2 \mathrm{H}), 7.40(\mathrm{t}, J=7.4 \mathrm{~Hz}, 2 \mathrm{H}), 7.31$ (t, $J=7.3 \mathrm{~Hz}, 2 \mathrm{H}), 6.55(\mathrm{~d}, J=7.0 \mathrm{~Hz}, 1 \mathrm{H}), 5.13(\mathrm{~d}, J=7.8 \mathrm{~Hz}, 1 \mathrm{H}), 4.99$ (s, 1H), 4.64-4.33 (m, $3 \mathrm{H}), 4.21(\mathrm{t}, J=5.9 \mathrm{~Hz}, 1 \mathrm{H}), 4.16-3.92(\mathrm{~m}, 1 \mathrm{H}), 3.20(\mathrm{dd}, J=12.4,6.3 \mathrm{~Hz}, 2 \mathrm{H}), 1.85-1.83(\mathrm{~m}$, 2H), 1.68-159 (m, 2H), $1.45(\mathrm{~s}, 9 \mathrm{H}), 1.43(\mathrm{~s}, 9 \mathrm{H}), 1.37(\mathrm{~d}, J=7.1 \mathrm{~Hz}, 3 \mathrm{H}), 1.28(\mathrm{~m}, 2 \mathrm{H}) .{ }^{13} \mathbf{C ~ N M R}$ $\left(\mathbf{1 0 0} \mathbf{M H z}, \mathbf{C D C l}_{3}\right) \delta 172.1,171.5,156.7,155.8,144.1,141.4,127.8,127.2,125.2,120.1,82.2$, 
80.2, 66.7, 54.4, 48.8, 47.4, 40.6, 32.3, 29.5, 28.4, 28.1, 22.6, 18.6. IR $v\left(\mathrm{~cm}^{-1}\right) 3321(\mathrm{~m}), 2978$ (m), 2935 (m), 1700 (s), 1667(s). HRMS (ESI) m/z: [M + H] Calcd for $\mathrm{C}_{33} \mathrm{H}_{46} \mathrm{~N}_{3} \mathrm{O}_{7}$ 596.3336; 596.3329 .

Phenyl ((S)-2-(((benzyloxy)carbonyl)amino)butanoyl)-L-phenylalaninate (10)<smiles>CCC(NC(=O)OCc1ccccc1)C(=O)NC(Cc1ccccc1)C(=O)OCc1ccccc1</smiles>

White solid, yield $103 \mathrm{mg}(89 \%), \mathrm{mp}=124-125^{\circ} \mathrm{C}, \mathrm{R}_{f} 0.4\left(9: 1, \mathrm{CH}_{2} \mathrm{Cl}_{2} / \mathrm{MeOH}\right) .{ }^{1} \mathbf{H}$ NMR (400 MHz, CDCl $) \delta$ 7.38-7.29 (m, 10H), 7.24-7.15 (m, 3H), $7.00(\mathrm{~s}, 2 \mathrm{H}), 6.31(\mathrm{~d}, J=7.7 \mathrm{~Hz}, 1 \mathrm{H})$, $5.25(\mathrm{~d}, J=7.6 \mathrm{~Hz}, 1 \mathrm{H}), 5.21-5.04(\mathrm{~m}, 4 \mathrm{H}), 4.92(\mathrm{~m}, 1 \mathrm{H}), 4.08(\mathrm{~d}, J=6.7 \mathrm{~Hz}, 1 \mathrm{H}), 3.11(\mathrm{t}, J=5.2$ Hz, 2H), 1.90-1.75 (m, 1H), 1.69-1.50 (m, 1H), 0.89 (t, $J=7.4$ Hz, 3H). ${ }^{13} \mathbf{C}$ NMR (100 MHz, $\left.\mathbf{C D C l}_{3}\right) \delta 171.2,156.1,136.3,136.2,135.5,135.1,129.4,128.8,128.7,128.3,128.2,127.3,67.5$, 67.2, 56.2, 53.2, 37.9, 26, 9.8. IR $\vee\left(\mathrm{cm}^{-1}\right) 3310(\mathrm{~s}), 2967$ (w), 1736 (s), 1663 (s). HRMS (ESI) $\mathrm{m} / \mathrm{z}:[\mathrm{M}+\mathrm{Na}]^{+}$Calcd for $\mathrm{C}_{28} \mathrm{H}_{30} \mathrm{~N}_{2} \mathrm{O}_{5} \mathrm{Na}$ 497.2052; found 497.2046.

\section{Tert-butyl(R)-2-((S)-4-((9H-xanthen-9-yl)amino)-2-((tert-butoxycarbonyl)amino)-4-} oxobutanamido)butanoate (11)

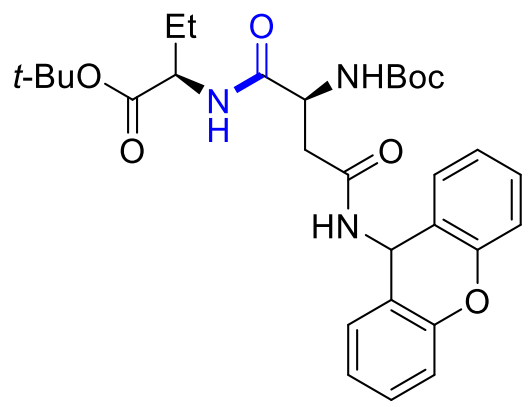

White solid, yield $121 \mathrm{mg}(87 \%), \mathrm{mp}=113-114{ }^{\circ} \mathrm{C}, \mathrm{R}_{f} 0.3\left(4: 1, \mathrm{CH}_{2} \mathrm{Cl}_{2} / \mathrm{CH}_{3} \mathrm{OH}\right) .{ }^{1} \mathbf{H}$ NMR (400 MHz, CDCl $) \delta 7.42(\mathrm{~d}, J=7.6 \mathrm{~Hz}, 2 \mathrm{H}), 7.36-7.18(\mathrm{~m}, 2 \mathrm{H}), 7.13-7.00(\mathrm{~m}, 4 \mathrm{H}), 6.47(\mathrm{~m}, 2 \mathrm{H})$, 6.17 (s, 1H), 4.52 (s, 1H), 4.33 (q, $J=8 \mathrm{~Hz}, 1 \mathrm{H}), 2.81$ (s, 1H), 2.77 (s, 1H), 2.59 (m, 1H), $1.84(\mathrm{~m}$, 1H), 1.67 (m, 1H), 1.48 (s, 9H), 1.44 (s, 9H), 0.88 (t, $J=7.5$ Hz, 3H). ${ }^{13} \mathbf{C}$ NMR (100 MHz, $\left.\mathbf{C D C l}_{3}\right) \delta 171,170.7,170.2,151.1,129.6,129.4,123.8,123.6,121,120.7,116.8,82.1,80.5,54.1$, 51.6, 44, 37.5, 28.4, 28.2, 25.5, 9.3. IR $v\left(\mathrm{~cm}^{-1}\right) 3310(\mathrm{~m}), 2967$ (w), 1741 (s), 1659 (s). HRMS (ESI) $\mathrm{m} / \mathrm{z}:[\mathrm{M}+\mathrm{Na}]^{+}$Calcd for $\mathrm{C}_{30} \mathrm{H}_{39} \mathrm{~N}_{3} \mathrm{O}_{7} \mathrm{Na}$ 576.2686; found 576.2680. 
Benzyl $\quad N^{6}$-(((9H-fluoren-9-yl)methoxy)carbonyl)- $N^{2}$-(tert-butoxycarbonyl)- $L$-lysyl- $L$ phenylalaninate (12)

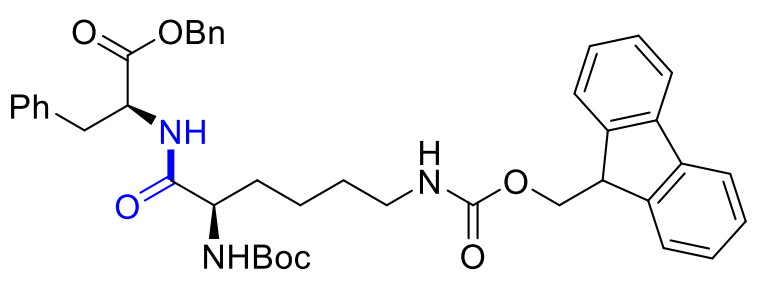

White solid, yield $164.4 \mathrm{mg}(93 \%), \mathrm{mp}=140-142{ }^{\circ} \mathrm{C}, \mathrm{R}_{f} 0.3\left(9: 1, \mathrm{CH}_{2} \mathrm{Cl}_{2} / \mathrm{CH}_{3} \mathrm{OH}\right) .{ }^{1} \mathbf{H}$ NMR (400 MHz, CDCl $\mathbf{l}_{3} \delta 7.72(\mathrm{~d}, J=7.6 \mathrm{~Hz}, 2 \mathrm{H}), 7.55$ (d, $\left.J=7.4 \mathrm{~Hz}, 2 \mathrm{H}\right), 7.44-7.11(\mathrm{~m}, 12 \mathrm{H}), 6.97$ (dd, $J=6.3,2.8 \mathrm{~Hz}, 2 \mathrm{H}), 6.40(\mathrm{~d}, J=8.0 \mathrm{~Hz}, 1 \mathrm{H}), 5.07$ (m, 2H), 4.99 (s, 1H), 4.85 (dd, $J=13.4,5.8$ $\mathrm{Hz}, 2 \mathrm{H}), 4.36$ (d, $J=6.6 \mathrm{~Hz}, 2 \mathrm{H}), 4.17$ (t, $J=6.7 \mathrm{~Hz}, 1 \mathrm{H}), 3.99$ (s, 1H), 3.29-2.96 (m, 4H), 1.83$1.67(\mathrm{~m}, 2 \mathrm{H}), 1.44(\mathrm{~m}, 2 \mathrm{H}), 1.38(\mathrm{~s}, 9 \mathrm{H}), 1.32-1.20(\mathrm{~m}, 2 \mathrm{H}) .{ }^{13} \mathbf{C}$ NMR (100 MHz, CDCl 3$) \delta$ $171.7,171.3,156.7,155.7,144.1,141.4,135.6,135.1,129.4,128.7,127.8,127.3,127.2$, 125.2 , $120.1,80.2,67.5,66.7,54.4,53.3,47.4,40.5,38,32.1,29.5,28.4,22.5$. IR $v\left(\mathrm{~cm}^{-1}\right) 3318(\mathrm{~m})$, 2930 (m), 2865 (w), 1696 (s), 1664 (s). HRMS (ESI) m/z: [M + Na $]^{+}$Calcd for $\mathrm{C}_{42} \mathrm{H}_{47} \mathrm{~N}_{3} \mathrm{O}_{7} \mathrm{Na}$ 728.3312 ; found 728.3308 .

Tert-butyl tosyl- $L$-alanyl- $L$-alaninate (13)<smiles>Cc1ccc(S(=O)(=O)N[C@@H](C)C(=O)N[C@@H](C)C(=O)OC(C)(C)C)cc1</smiles>

Viscous oil, yield $78 \mathrm{mg}(84 \%), \mathrm{R}_{f} 0.3$ (1:1, hexane/ethyl acetate). ${ }^{1} \mathbf{H}$ NMR (400 $\left.\mathbf{M H z}, \mathbf{C D C l}_{3}\right)$ $\delta 7.74(\mathrm{~d}, J=8.2 \mathrm{~Hz}, 2 \mathrm{H}), 7.29(\mathrm{~d}, J=8.3 \mathrm{~Hz}, 2 \mathrm{H}), 6.42(\mathrm{~d}, J=6.9 \mathrm{~Hz}, 1 \mathrm{H}), 5.20(\mathrm{~d}, J=8.0 \mathrm{~Hz}$, 1H), 4.39-4.15 (m, 1H), 3.85-3.70 (m, 1H), 2.41 (s, 3H), $1.45(\mathrm{~s}, 9 \mathrm{H}), 1.29$ (d, J=7.0 Hz, 3H), $1.20(\mathrm{~d}, J=7.0 \mathrm{~Hz}, 3 \mathrm{H}) .{ }^{13} \mathbf{C}$ NMR (100 MHz, $\left.\mathbf{C D C l}_{3}\right) \delta 171.7,171,143.8,136.9,129.8,127.4$, 82.4, 52.4, 49, 28, 21.6, 19.6, 18.3. IR v ( $\left.\mathrm{cm}^{-1}\right) 3310$ (m), 2967 (w), 1741 (s), 1659 (s). HRMS (ESI) m/z: $[\mathrm{M}+\mathrm{Na}]^{+}$Calcd for $\mathrm{C}_{17} \mathrm{H}_{26} \mathrm{~N}_{2} \mathrm{O}_{5} \mathrm{SNa} 393.1460$; found 393.1455.

Methyl tosyl- $L$-alanyl- $L$-phenylalaninate (14) 


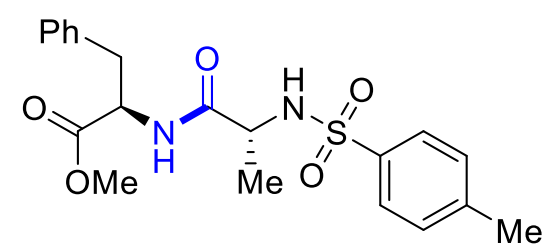

Viscous oil, yield $91 \mathrm{mg}$ (90\%), $\mathrm{R}_{f} 0.3$ (1:1, hexanes/ethyl acetate). ${ }^{1} \mathbf{H}$ NMR (400 $\mathbf{~ M H z}, \mathbf{C D C l}_{3}$ ) $\delta 7.77(\mathrm{~d}, J=8.2 \mathrm{~Hz}, 2 \mathrm{H}), 7.35-7.27(\mathrm{~m}, 4 \mathrm{H}), 7.08-7.00(\mathrm{~m}, 2 \mathrm{H}), 6.82(\mathrm{~d}, J=7.8 \mathrm{~Hz}, 1 \mathrm{H}), 5.79(\mathrm{~d}$, $J=7.8 \mathrm{~Hz}, 1 \mathrm{H}), 4.74(\mathrm{q}, J=8.0 \mathrm{~Hz}, 1 \mathrm{H}), 3.92-3.78(\mathrm{~m}, 1 \mathrm{H}), 3.71(\mathrm{~s}, 3 \mathrm{H}), 3.03(\mathrm{dd}, J=5.9,3.0$ $\mathrm{Hz}, 2 \mathrm{H}), 2.42(\mathrm{~s}, 3 \mathrm{H}), 1.23(\mathrm{~d}, J=7.1 \mathrm{~Hz}, 3 \mathrm{H}) .{ }^{13} \mathbf{C} \mathbf{N M R}\left(\mathbf{1 0 0} \mathbf{M H z}, \mathbf{C D C l}_{3}\right) \delta$ 171.5, 171.2, 143.8, 136.8, 135.7, 129.8, 129.3, 128.6, 127.3, 127.2, 53.5, 52.4, 52.4, 37.8, 21.6, 19.4. IR v(cm1) 3325 (m), 2955 (w), 1700 (s), 1637 (s). HRMS (ESI) m/z: [M + Na] ${ }^{+}$Calcd for $\mathrm{C}_{20} \mathrm{H}_{24} \mathrm{~N}_{2} \mathrm{O}_{5} \mathrm{SNa}$ 427.1304; found 427.1301.

Tert-butyl (R)-2-((R)-3-(4-((((2-bromobenzyl)oxy)carbonyl)oxy)phenyl)-2-((tertbutoxycarbonyl)amino)propanamido)butanoate (15)

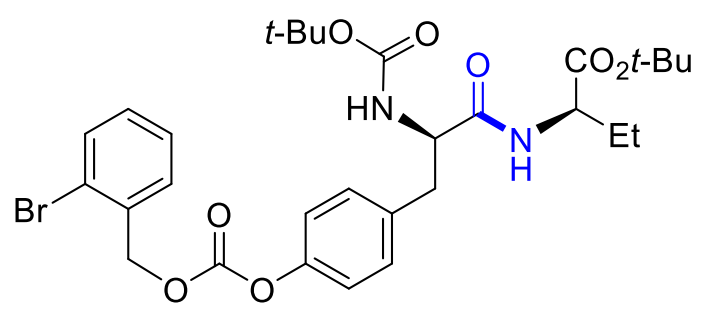

White solid, yield $152.5 \mathrm{mg}(96 \%), \mathrm{mp}=109-110{ }^{\circ} \mathrm{C}, \mathrm{R}_{f} 0.3\left(9: 1, \mathrm{CH}_{2} \mathrm{Cl}_{2} / \mathrm{CH}_{3} \mathrm{OH}\right) .{ }^{1} \mathbf{H}$ NMR (400 MHz, CDCl $) \delta 7.62(\mathrm{~d}, J=8.0 \mathrm{~Hz}, 1 \mathrm{H}), 7.52(\mathrm{~d}, J=7.7 \mathrm{~Hz}, 1 \mathrm{H}), 7.37(\mathrm{t}, J=7.5 \mathrm{~Hz}, 1 \mathrm{H}), 7.24$ (d, $J=6.8 \mathrm{~Hz}, 3 \mathrm{H}), 7.15$ (d, $J=8.5 \mathrm{~Hz}, 2 \mathrm{H}), 6.39$ (d, $J=7.6 \mathrm{~Hz}, 1 \mathrm{H}), 5.38(\mathrm{~s}, 2 \mathrm{H}), 5.00(\mathrm{~s}, 1 \mathrm{H})$, 4.58-4.34 (m, 2H), 3.33-2.82 (m, 2H), 1.90-1.69 (m, 1H), 1.63-1.56 (m, 1H), 1.46 (s, 9H), 1.42 (s, 9H), $0.76(\mathrm{t}, J=7.5 \mathrm{~Hz}, 3 \mathrm{H}) .{ }^{13} \mathbf{C}$ NMR (100 MHz, $\left.\mathbf{C D C l}_{3}\right) \delta$ 171.1, 170.7, 155.3, 153.3, 149.9, $134.8,134.2,132.9,130.3,130.2,130.1,127.6,123.4,121,81.9,80,69.5,55.7,53.6,37.8,28.2$, 27.9, 25.4, 9.1. IR $v\left(\mathrm{~cm}^{-1}\right) 3315$ (m), 2942 (m), 2839 (m), 1733 (s), 1662 (s). HRMS (ESI) m/z: $[\mathrm{M}+\mathrm{Na}]^{+}$Calcd for $\mathrm{C}_{30} \mathrm{H}_{39} \mathrm{BrN}_{2} \mathrm{O}_{8} \mathrm{Na} 657.1787$; found 657.1786 .

Methyl((R)-3-(4-((((2-bromobenzyl)oxy)carbonyl)oxy)phenyl)-2-((tertbutoxycarbonyl)amino)propanoyl)-L-phenylalaninate (16) 


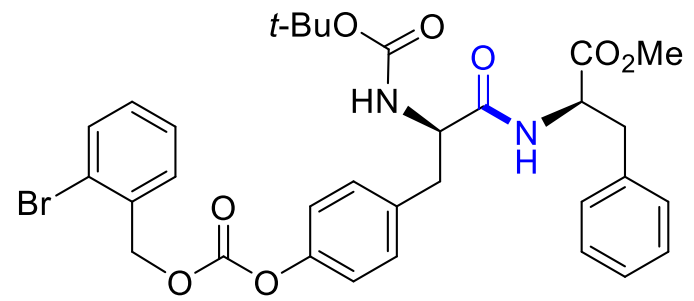

White solid, yield $152.5 \mathrm{mg}(93 \%), \mathrm{mp}=109-110{ }^{\circ} \mathrm{C}, \mathrm{R}_{f} 0.5\left(9: 1, \mathrm{CH}_{2} \mathrm{Cl}_{2} / \mathrm{CH}_{3} \mathrm{OH}\right) .{ }^{1} \mathbf{H}$ NMR (500 MHz, $\left.\mathbf{C D C l}_{3}\right) \delta 7.62(\mathrm{~d}, J=8.0 \mathrm{~Hz}, 1 \mathrm{H}), 7.51(\mathrm{~d}, J=7.5 \mathrm{~Hz}, 1 \mathrm{H}), 7.36(\mathrm{t}, J=7.5 \mathrm{~Hz}, 1 \mathrm{H}), 7.31-$ $7.18(\mathrm{~m}, 6 \mathrm{H}), 7.13(\mathrm{~d}, J=7.2 \mathrm{~Hz}, 2 \mathrm{H}), 7.02(\mathrm{~d}, J=7.4 \mathrm{~Hz}, 2 \mathrm{H}), 6.32(\mathrm{~d}, J=7.0 \mathrm{~Hz}, 1 \mathrm{H}), 5.37$ (s, 2H), 4.95 (s, 1H), 4.79 (d, $J=6.1 \mathrm{~Hz}, 1 \mathrm{H}), 4.33$ (s, 1H), 3.69 (s, 3H), 3.07 (m, 4H), 1.42 (s, 9H). ${ }^{13} \mathrm{C}$ NMR (100 MHz, $\left.\mathbf{C D C l}_{3}\right) \delta$ 171.5, 170.7, 155.4, 153.5, 150.2, 135.7, 134.5, 134.3, 133.1, 130.7, 130.6, 130.3, 130.2, 129.3, 128.7, 127.8, 127.3, 121.2, 77.5, 77.2, 76.8, 70.7, 69.8, 55.7, 53.4, 52.5, 38, 37.7, 28.4. IR $v\left(\mathrm{~cm}^{-1}\right) 3315(\mathrm{~m}), 2926$ (m), 1746 (s), 1509 (s). HRMS (ESI) m/z: $[\mathrm{M}+\mathrm{H}]^{+}$Calcd for $\mathrm{C}_{32} \mathrm{H}_{36} \mathrm{BrN}_{2} \mathrm{O}_{8} 655.1655$; found 655.1652 .

\section{Benzyl (isoquinoline-1-carbonyl)- $L$-phenylalaninate (17)}<smiles>O=C(N[C@@H](Cc1ccccc1)C(=O)OCc1ccccc1)c1nccc2ccccc12</smiles>

White solid, yield $91.5 \mathrm{mg}(89 \%), \mathrm{mp}=104-105^{\circ} \mathrm{C}, \mathrm{R}_{f} 0.2$ (6:4, hexanes/ethyl acetate). ${ }^{1} \mathbf{H}$ NMR (400 MHz, $\left.\mathbf{C D C l}_{3}\right) \delta 9.54(\mathrm{~d}, J=8.4 \mathrm{~Hz}, 1 \mathrm{H}), 8.74(\mathrm{~d}, J=8.1 \mathrm{~Hz}, 1 \mathrm{H}), 8.47$ (d, $\left.J=5.5 \mathrm{~Hz}, 1 \mathrm{H}\right)$, $7.84(\mathrm{~d}, J=7.8 \mathrm{~Hz}, 1 \mathrm{H}), 7.79(\mathrm{~d}, J=5.5 \mathrm{~Hz}, 1 \mathrm{H}), 7.75-7.60(\mathrm{~m}, 2 \mathrm{H}), 7.40-7.28(\mathrm{~m}, 5 \mathrm{H}), 7.28-7.09$ (m, 5H), 5.47-4.96 (m, 3H), 3.57-3.14 (m, 2H). ${ }^{13} \mathbf{C}$ NMR (100 MHz, CDCl $) \delta 171.5,165.8$, $147.5,140.5,137.5,136.1,135.4,130.5,129.5,128.8,128.7,128.6,128.5,127.7,127.1,126.9$, 124.6, 77.5, 77.2, 76.8, 67.3, 53.7, 38.4. IR v ( $\left.\mathrm{cm}^{-1}\right) 3059(\mathrm{w}), 3059$ (w), 1738 (s), $1668(\mathrm{~s})$. HRMS (ESI) $\mathrm{m} / \mathrm{z}$ : $[\mathrm{M}+\mathrm{H}]^{+}$Calcd for $\mathrm{C}_{26} \mathrm{H}_{23} \mathrm{~N}_{2} \mathrm{O}_{3}$ 411.1709; found 411.1706. 


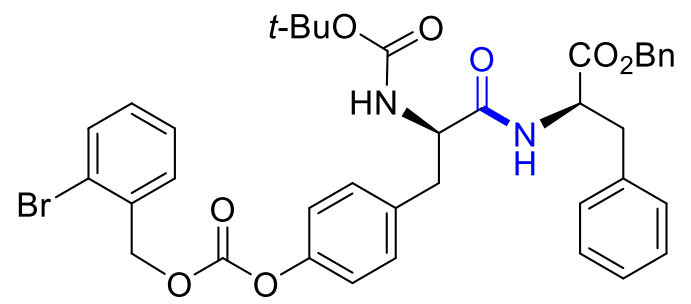

White solid, yield $174 \mathrm{mg}(95 \%), \mathrm{mp}=129-130{ }^{\circ} \mathrm{C}, \mathrm{R}_{f} 0.5\left(9: 1, \mathrm{CH}_{2} \mathrm{Cl}_{2} / \mathrm{CH}_{3} \mathrm{OH}\right) .{ }^{1} \mathbf{H}$ NMR (400 MHz, $\left.\mathbf{C D C l}_{3}\right) \delta 7.47$ (d, $\left.J=8.0 \mathrm{~Hz}, 1 \mathrm{H}\right), 7.37$ (d, $\left.J=7.5 \mathrm{~Hz}, 1 \mathrm{H}\right), 7.22(\mathrm{~d}, J=4.7 \mathrm{~Hz}, 4 \mathrm{H}), 7.17-$ $7.00(\mathrm{~m}, 8 \mathrm{H}), 6.97$ (s, 2H), $6.80(\mathrm{~d}, J=3.9 \mathrm{~Hz}, 2 \mathrm{H}), 6.24(\mathrm{~s}, 1 \mathrm{H}), 5.22(\mathrm{~s}, 2 \mathrm{H}), 4.97(\mathrm{~s}, 2 \mathrm{H}), 4.84$ $(\mathrm{d}, J=5.9 \mathrm{~Hz}, 1 \mathrm{H}), 4.68(\mathrm{~d}, J=6.5 \mathrm{~Hz}, 1 \mathrm{H}), 4.19(\mathrm{~s}, 1 \mathrm{H}), 3.01-2.80(\mathrm{~m}, 4 \mathrm{H}), 1.26(\mathrm{~s}, 9 \mathrm{H}) .{ }^{13} \mathrm{C}$ NMR (100 MHz, $\left.\mathbf{C D C l}_{3}\right) \delta 170.9,170.7,155.4,153.5,150.2,135.6,135.1,134.6,134.3,133.1$, 130.7, 130.5, 130.3, 130.2, 129.5, 129.4, 128.9, 128.7, 127.8, 127.2, 123.4, 121.2, 80.3, 69.7, 67.4, 55.6, 53.4, 38, 37.7, 28.4. IR $v\left(\mathrm{~cm}^{-1}\right) 3289$ (m), 2914 (m), 1732 (s), 1661 (s). HRMS (ESI) m/z: $[\mathrm{M}+\mathrm{H}]^{+}$Calcd for $\mathrm{C}_{38} \mathrm{H}_{40} \mathrm{BrN}_{2} \mathrm{O}_{8}$ 731.1968; found 731.1965.

Tert-butyl $\quad(R)-2-((S)-2-((((9 H$-fluoren-9-yl)methoxy)carbonyl)amino)-3-(1-trityl-1Himidazol-4-yl)propanamido)butanoate (19)

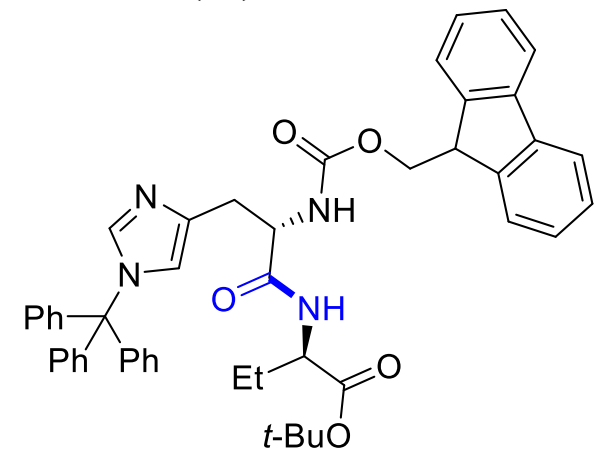

White solid, yield $173 \mathrm{mg}(91 \%), \mathrm{mp}=81-82{ }^{\circ} \mathrm{C}, \mathrm{R}_{f} 0.3$ (1:1, hexanes/ethyl acetate). ${ }^{1} \mathrm{H}$ NMR $\left(400 \mathrm{MHz}, \mathbf{C D C l}_{3}\right) \delta 7.65(\mathrm{~d}, J=7.5 \mathrm{~Hz}, 2 \mathrm{H}), 7.51(\mathrm{~d}, J=6.7 \mathrm{~Hz}, 2 \mathrm{H}), 7.28(\mathrm{~m}, 4 \mathrm{H}), 7.24-7.13$ (m, 11H), $6.99(\mathrm{~m}, 6 \mathrm{H}), 6.68(\mathrm{~s}, 1 \mathrm{H}), 6.56(\mathrm{~s}, 1 \mathrm{H}), 4.43(\mathrm{dd}, J=12.3,5.9 \mathrm{~Hz}, 1 \mathrm{H}), 4.30(\mathrm{dd}, J=$ 13.2, $5.9 \mathrm{~Hz}, 1 \mathrm{H}), 4.26-4.18(\mathrm{~m}, 2 \mathrm{H}), 4.17-4.08(\mathrm{~m}, 1 \mathrm{H}), 3.05-2.78(\mathrm{~m}, 2 \mathrm{H}), 1.59(\mathrm{~m}, 2 \mathrm{H}), 1.33(\mathrm{~s}$, 9H), $0.72(\mathrm{t}, J=7.1 \mathrm{~Hz}, 3 \mathrm{H}) .{ }^{13} \mathbf{C}$ NMR (100 MHz, CDCl $\left.\mathbf{3}\right) \delta 171.2,170.9,156.4,144.1,142.5$, $141.4,138.7,137,130,128.2,127.8,127.2,125.5,125.4,120.0,119.7,82,77.5,77.2,76.8,75.5$, 67.4, 55.4, 54, 47.3, 30.7, 28.2, 25.7, 9.3. IR $v\left(\mathrm{~cm}^{-1}\right) 3289(\mathrm{~s}), 2972(\mathrm{~m}), 2871(\mathrm{w}), 1685(\mathrm{~s}), 1653$ (s). HRMS (APCI) m/z: [M+ H] $]^{+}$Calcd for $\mathrm{C}_{48} \mathrm{H}_{49} \mathrm{~N}_{4} \mathrm{O}_{5} 761.3697$, found 761.3700 . 
$N$-(3,5-dimethylphenyl)-2-(4-hydroxyphenyl)acetamide ${ }^{3,4}(20)$

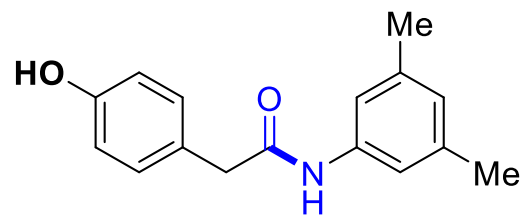

Off-white solid, yield $1.42 \mathrm{~g}(86 \%), \mathrm{mp}=183-184{ }^{\circ} \mathrm{C}\left(\text { lit. } 183-185{ }^{\circ} \mathrm{C}\right)^{4} \mathrm{R}_{f} 0.4(2: 3$, hexanes/ethyl acetate). ${ }^{1}$ H NMR (400 MHz, DMSO-d $) \delta 9.89$ (s, 1H), 9.24 (s, 1H), 7.20 (s, 2H), 7.10 (d, J= $7.9 \mathrm{~Hz}, 2 \mathrm{H}), 6.75-6.64(\mathrm{~m}, 3 \mathrm{H}), 3.45(\mathrm{~s}, 2 \mathrm{H}), 2.21(\mathrm{~s}, 6 \mathrm{H}) .{ }^{13} \mathrm{C}$ NMR (100 MHz, DMSO-d $) \delta$ 207.2, 193.7, 176.8, 175.3, 167.6, 163.9, 162.3, 154.5, 152.7, 80.3, 58.8.

Methyl ((S)-2-(((benzyloxy)carbonyl)amino)butanoyl)- $L$-phenylalaninate (21)<smiles>CC[C@H](NC(=O)OCc1ccccc1)C(=O)N[C@@H](Cc1ccccc1)C(=O)OC</smiles>

White solid, yield $87 \mathrm{mg}(87 \%), \mathrm{mp}=114-115^{\circ} \mathrm{C}, \mathrm{R}_{f} 0.3$ (1:1, hexanes/ethyl acetate). ${ }^{1} \mathbf{H}$ NMR $\left(400 \mathrm{MHz}, \mathbf{C D C l}_{3}\right) \delta$ 7.46-7.25 (m, 8H), $7.13(\mathrm{~d}, J=6.9 \mathrm{~Hz}, 2 \mathrm{H}), 6.46(\mathrm{~d}, J=7.4 \mathrm{~Hz}, 1 \mathrm{H}), 5.36$ (d, $J=7.4 \mathrm{~Hz}, 1 \mathrm{H}), 5.20-5.07$ (m, 2H), $4.92(\mathrm{dd}, J=13.2,6.1 \mathrm{~Hz}, 1 \mathrm{H}), 4.16(\mathrm{dd}, J=13.4,6.5 \mathrm{~Hz}$, 1H), $3.76(\mathrm{~s}, 3 \mathrm{H}), 3.16(\mathrm{~m}, 2 \mathrm{H}), 1.88(\mathrm{~m}, 1 \mathrm{H}), 1.66(\mathrm{~m}, 1 \mathrm{H}), 0.95(\mathrm{t}, J=7.4 \mathrm{~Hz}, 3 \mathrm{H}) .{ }^{13} \mathbf{C}$ NMR (100 MHz, $\left.\mathbf{C D C l}_{3}\right) \delta 171.8,171.2,156.2,136.3,135.7,129.4,128.7,128.7,128.3,128.2,127.3$, 67.2, 56.1, 53.2, 52.5, 38, 26, 9.8. IR $v\left(\mathrm{~cm}^{-1}\right) 3067(\mathrm{~m}), 2967$ (w), 1741 (s), 1659 (s). HRMS (ESI) $\mathrm{m} / \mathrm{z}:[\mathrm{M}+\mathrm{Na}]^{+}$Calcd for $\mathrm{C}_{22} \mathrm{H}_{26} \mathrm{O}_{5} \mathrm{~N}_{2} \mathrm{Na} 421.1739$; found 421.1735 .

Tert-butyl(S)-2-((R)-6-((((9H-fluoren-9-yl)methoxy)carbonyl)amino)-2-((tertbutoxycarbonyl)amino)hexanamido)butanoate (22)

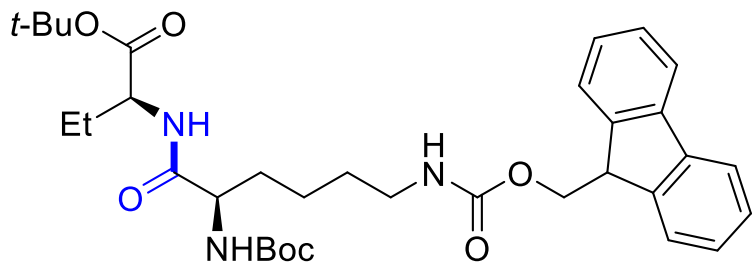

White solid, yield $130 \mathrm{mg}(85 \%), \mathrm{mp}=89-90{ }^{\circ} \mathrm{C}, \mathrm{R}_{f} 0.3\left(9: 1, \mathrm{CH}_{2} \mathrm{Cl}_{2} / \mathrm{CH}_{3} \mathrm{OH}\right) .{ }^{1} \mathbf{H}$ NMR (400 MHz, $\left.\mathbf{C D C l}_{3}\right) \delta 7.76(\mathrm{~d}, J=7.5 \mathrm{~Hz}, 2 \mathrm{H}), 7.60(\mathrm{~d}, J=7.2 \mathrm{~Hz}, 2 \mathrm{H}), 7.40(\mathrm{t}, J=7.4 \mathrm{~Hz}, 2 \mathrm{H}), 7.31$ $(\mathrm{m}, 2 \mathrm{H}), 6.62(\mathrm{~s}, 1 \mathrm{H}), 5.09(\mathrm{~s}, 1 \mathrm{H}), 4.90(\mathrm{~s}, 1 \mathrm{H}), 4.51-4.30(\mathrm{~m}, 3 \mathrm{H}), 4.21(\mathrm{t}, J=6.8 \mathrm{~Hz}, 1 \mathrm{H}), 4.11$ 
(s, 1H), $3.20(\mathrm{~d}, J=6.3 \mathrm{~Hz}, 2 \mathrm{H}), 1.87(\mathrm{~m}, 2 \mathrm{H}), 1.79-1.59(\mathrm{~m}, 2 \mathrm{H}), 1.59-1.51(\mathrm{~m}, 2 \mathrm{H}), 1.46(\mathrm{~s}, 9 \mathrm{H})$,

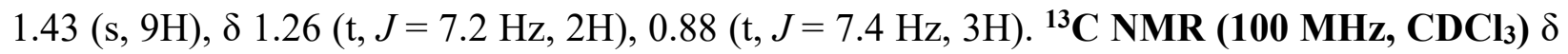
171.6, 171.4, 156.7, 155.8, 144.1, 141.4, 127.8, 127.2, 125.2, 120.1, 82.2, 80.26, 66.7, 54.6, 53.7, 47.4, 40.6, 32.2, 29.6, 28.4, 28.1, 25.7, 22.7, 9.3. IR v (cm-1) 3314 (m), 2927 (m), 2865 (w), 1696 (s), 1659 (s). HRMS (ESI) m/z: [M + Na $]^{+}$Calcd for $\mathrm{C}_{34} \mathrm{H}_{47} \mathrm{~N}_{3} \mathrm{O}_{7} \mathrm{Na}$ 632.3312; found 632.3302.

\section{Methyl benzoyl- $L$-phenylalaninate ${ }^{5}(23)$}

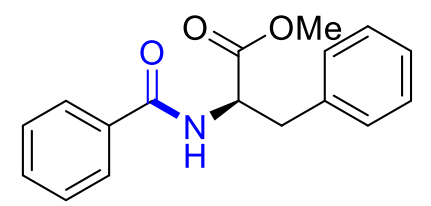

White solid, yield $69 \mathrm{mg}(93 \%), \mathrm{mp}=80-81^{\circ} \mathrm{C}\left(\text { lit. } 80-81{ }^{\circ} \mathrm{C}\right)^{5}, \mathrm{R}_{f} 0.3(7: 3$, hexanes/ethyl acetate). ${ }^{1}$ H NMR (400 MHz, CDCl 3$) \delta 7.73(\mathrm{~d}, J=7.7 \mathrm{~Hz}, 2 \mathrm{H}), 7.50(\mathrm{t}, J=7.2 \mathrm{~Hz}, 1 \mathrm{H}), 7.41$ (t, $J=7.5$ $\mathrm{Hz}, 2 \mathrm{H}), 7.33-7.19(\mathrm{~m}, 3 \mathrm{H}), 7.15$ (d, $J=7.1 \mathrm{~Hz}, 2 \mathrm{H}), 6.68$ (d, $J=7.0 \mathrm{~Hz}, 1 \mathrm{H}), 5.10$ (q, $J=5.9 \mathrm{~Hz}$, 1H), 3.76 (s, 3H), 3.27 (m, 2H). ${ }^{13} \mathbf{C}$ NMR (100 MHz, $\left.\mathbf{C D C l}_{3}\right) \delta 172.1,166.9,135.9,133.9,131.8$, $129.4,128.7,128.7,127.2,127.1,53.6,52.5,37.9$.

Methyl $\quad N^{2}$-(tert-butoxycarbonyl)- $N^{4}$-(9H-xanthen-9-yl)- $L$-asparaginyl- $L$-phenylalaninate (24)

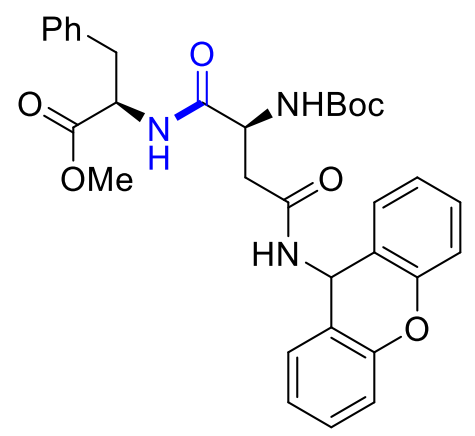

White solid, yield $135 \mathrm{mg}$ (94\%), $\mathrm{mp}=198.5-200{ }^{\circ} \mathrm{C}, \mathrm{R}_{f} 0.3$ (9:1, $\left.\mathrm{CH}_{2} \mathrm{Cl}_{2} / \mathrm{CH}_{3} \mathrm{OH}\right) .{ }^{1} \mathbf{H}$ NMR (400 MHz, $\left.\mathbf{C D C l}_{3}\right) \delta 7.42(\mathrm{~m}, 2 \mathrm{H}), 7.34-7.20(\mathrm{~m}, 5 \mathrm{H}), 7.18(\mathrm{~d}, J=7.0 \mathrm{~Hz}, 2 \mathrm{H}), 7.13-6.92(\mathrm{~m}, 4 \mathrm{H})$, $6.47(\mathrm{~d}, J=9.1 \mathrm{~Hz}, 1 \mathrm{H}), 6.36(\mathrm{~d}, J=9.8 \mathrm{~Hz}, 1 \mathrm{H}), 6.11(\mathrm{~s}, 1 \mathrm{H}), 4.77$ (dd, $J=13.7,6.0 \mathrm{~Hz}, 1 \mathrm{H})$, $4.51(\mathrm{~s}, 1 \mathrm{H}), 3.66(\mathrm{~s}, 3 \mathrm{H}), 3.57-3.54(\mathrm{~m}, 1 \mathrm{H}), 3.10(\mathrm{~d}, J=5.9 \mathrm{~Hz}, 2 \mathrm{H}), 2.81(\mathrm{~d}, J=13.3 \mathrm{~Hz}, 1 \mathrm{H})$, $2.57(\mathrm{dd}, J=15.5,6.3 \mathrm{~Hz}, 1 \mathrm{H}), 1.42$ (s, 9H). ${ }^{13} \mathbf{C}$ NMR (100 MHz, $\left.\mathbf{C D C l}_{3}\right) \delta 171.5,171.2,170.4$, $151.1,135.9,129.4,129.3,128.7,127.2$, 123.7, 123.6, 120.8, 116.7, 80.4, 70.7, 53.7, 52.4, 51.2, 
44, 38, 28.4, 28.3. IR v (cm-1) $3304(\mathrm{~m}), 2926(\mathrm{~m}), 2865(\mathrm{w}), 1641$ (s), 1527 (s). HRMS (ESI) m/z: $[\mathrm{M}+\mathrm{Na}]^{+}$Calcd for $\mathrm{C}_{32} \mathrm{H}_{35} \mathrm{~N}_{3} \mathrm{O}_{7} \mathrm{Na}$ 596.2373; found 596.2367.

\section{Benzyl acetyl- $L$-methionyl- $L$-phenylalaninate (25)}<smiles>CSCC[C@H](NC(C)=O)C(=O)N[C@@H](Cc1ccccc1)C(=O)OCc1ccccc1</smiles>

Viscous oil yield $90 \mathrm{mg}(84 \%), \mathrm{R}_{f} 0.3$ (9:1, $\left.\mathrm{CH}_{2} \mathrm{Cl}_{2} / \mathrm{CH}_{3} \mathrm{OH}\right) .{ }^{1} \mathbf{H}$ NMR (400 MHz, CDCl $) \delta 7.30$ (m, 8H), 7.09-6.97 (m, 2H), 6.70-6.48 (m, 1H), $6.18(\mathrm{~d}, J=7.7 \mathrm{~Hz}, 1 \mathrm{H}), 5.16(\mathrm{~m}, 2 \mathrm{H}), 4.87$ (d, $=7.2 \mathrm{~Hz}, 1 \mathrm{H}), 4.56(\mathrm{q}, J=6.8 \mathrm{~Hz}, 1 \mathrm{H}), 3.35-2.90(\mathrm{~m}, 2 \mathrm{H}), 2.59-2.37(\mathrm{~m}, 2 \mathrm{H}), 2.32-2.21(\mathrm{~m}, 1 \mathrm{H})$, $2.03(\mathrm{~d}, J=7.3 \mathrm{~Hz}, 3 \mathrm{H}), 1.96(\mathrm{~d}, J=7.8 \mathrm{~Hz}, 3 \mathrm{H}), 1.92-1.78(\mathrm{~m}, 1 \mathrm{H}) .{ }^{13} \mathbf{C}$ NMR (100 MHz, CDCl $)$ $\delta 171,170.2,170,135.7,135.5,135.1,129.2,128.8,127.3,67.5,53.3,52.3,52,37.7,31.6,30$, 23.3, 15.3, 14.8. IR $v\left(\mathrm{~cm}^{-1}\right) 3274(\mathrm{~m}), 3064(\mathrm{~m}), 2920$ (w), 1742 (m), 1644 (s). HRMS (ESI) m/z: $[\mathrm{M}+\mathrm{Na}]^{+}$Calcd for $\mathrm{C}_{23} \mathrm{H}_{28} \mathrm{~N}_{2} \mathrm{O}_{4} \mathrm{SNa} 451.1667$; found 451.1664 .

\section{Benzyl benzoyl- $L$-phenylalaninate ${ }^{6}(26)$}<smiles>O=C(N[C@@H](Cc1ccccc1)C(=O)OCc1ccccc1)c1ccccc1</smiles>

White solid, yield $86 \mathrm{mg}(95 \%), \mathrm{mp}=98-99{ }^{\circ} \mathrm{C}$ (lit. 98.5-99 $\left.{ }^{\circ} \mathrm{C}\right)^{6} . \mathrm{R}_{f} 0.4(7: 3$, hexanes/ethyl acetate). ${ }^{1} \mathbf{H}$ NMR (400 MHz, $\left.\mathbf{C D C l}_{3}\right) \delta 7.72(\mathrm{dd}, J=8.0,0.9 \mathrm{~Hz}, 2 \mathrm{H}), 7.51$ (t, $\left.J=7.4 \mathrm{~Hz}, 1 \mathrm{H}\right)$, 7.46-7.31 (m, 7H), 7.25-7.19 (m, $3 \mathrm{H}), 7.03(\mathrm{dd}, J=6.5,2.8 \mathrm{~Hz}, 2 \mathrm{H}), 6.62(\mathrm{~d}, J=7.4 \mathrm{~Hz}, 1 \mathrm{H})$,

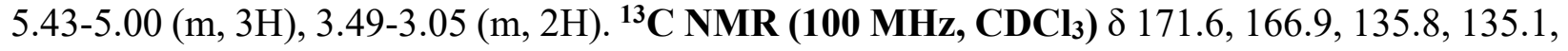
$134,131.9,129.5,128.8,128.8,128.7,128.7,127.2,127.1,77.5,77.2,76.8,67.5,53.6,38$.

Benzyl (S)-(3-methyl-1-oxo-1-(phenylamino)butan-2-yl)carbamate $(27)^{7,8}$ 


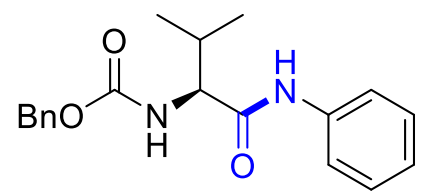

White solid, yield $75 \mathrm{mg}(92 \%), \mathrm{mp}=182-183{ }^{\circ} \mathrm{C}$ (lit. $\left.182-184{ }^{\circ} \mathrm{C}\right), \mathrm{R}_{f} 0.3(1: 1$, hexanes/ethyl acetate). ${ }^{1} \mathbf{H}$ NMR (400 MHz, $\left.\mathbf{C D C l}_{3}\right) \delta 7.89(\mathrm{~s}, 1 \mathrm{H}), 7.41(\mathrm{~d}, J=7.9 \mathrm{~Hz}, 2 \mathrm{H}), 7.29-7.17(\mathrm{~m}, 7 \mathrm{H})$, 7.03 (t, $J=7.5 \mathrm{~Hz}, 1 \mathrm{H}), 5.40$ (d, $J=8.1 \mathrm{~Hz}, 1 \mathrm{H}), 5.04(\mathrm{~s}, 2 \mathrm{H}), 4.07-4.00(\mathrm{~m}, 1 \mathrm{H}), 2.32-2.07$ (m, $1 \mathrm{H}), 0.96(\mathrm{~d}, J=6.8 \mathrm{~Hz}, 3 \mathrm{H}), 0.92(\mathrm{~d}, J=6.8 \mathrm{~Hz}, 3 \mathrm{H})$. 


\section{REFERENCES}

1. Brals, J.; Smith, J. D.; Ibrahim, F.; Gallou, F.; Handa, S. Micelle-Enabled Palladium Catalysis for Convenient Sp2-Sp3 Coupling of Nitroalkanes with Aryl Bromides in Water Under Mild Conditions. ACS Catal. 2017, 7 (10), 7245-7250.

2. Song, W.; Ma, L.; Hu, L. HClO4-SiO2 as an Efficient and Recyclable Catalyst for the Synthesis of Amide Derivatives. Synth. Commun. 2011, 41 (21), 3186-3196.

3. Anderson, J. E.; Davis, R.; Fitzgerald, R. N.; Haberman, J. M. Selective Phenol Alkylation: An Improved Preparation of Efaproxiral. Synth. Commun. 2006, 36 (15), 2129-2133.

4. Phelps Grella, M.; Danso-Danquah, R.; Safo, M. K.; Joshi, G. S.; Kister, J.; Marden, M.; Hoffman, S. J.; Abraham, D. J. Synthesis and Structure-Activity Relationships of Chiral Allosteric Modifiers of Hemoglobin. J. Med. Chem. 2000, 43 (25), 4726-4737.

5. Thalhammer, A.; Mecinović, J.; Schofield, C. J. Triflic Anhydride-Mediated Synthesis of Oxazoles. Tetrahedron Lett. 2009, 50 (9), 1045-1047.

6. Mandai, H.; Hongo, K.; Fujiwara, T.; Fujii, K.; Mitsudo, K.; Suga, S. Dynamic Kinetic Resolution of Azlactones by a Chiral N,N-Dimethyl-4-Aminopyridine Derivative Containing a 1,1'-Binaphthyl Unit: Importance of Amide Groups. Org. Lett. 2018, 20 (16), $4811-4814$.

7. Bai, J.; Zambroń, B. K.; Vogel, P. Amides in One Pot from Carboxylic Acids and Amines via Sulfinylamides. Org. Lett. 2014, 16 (2), 604-607.

8. Kucherenko, A. S.; Siyutkin, D. E.; Dashkin, R. R.; Zlotin, S. G. Organocatalysis of Asymmetric Aldol Reaction in Water: Comparison of Catalytic Properties of (S)-Valine and (S)-Proline Amides. Russ. Chem. Bull. 2013, 62 (4), 1010-1015. 


\section{NMR SPECTRA}
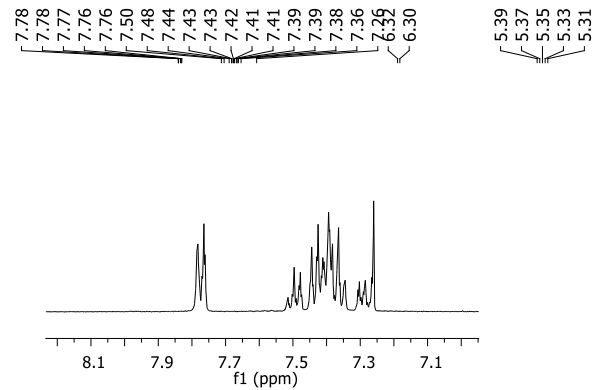<smiles>CC(NC(=O)c1ccccc1)c1ccccc1</smiles>

3

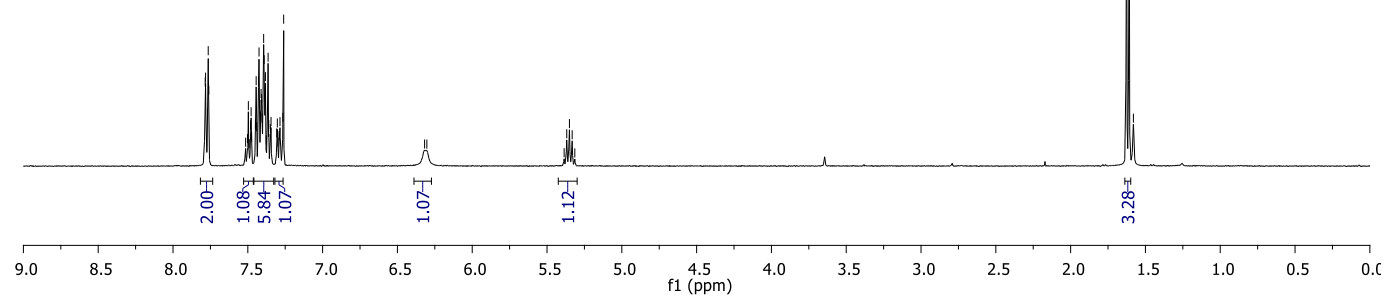

${ }^{1} \mathrm{H}$ NMR Spectrum of 3 in $\mathrm{CDCl}_{3}(400 \mathrm{MHz})$

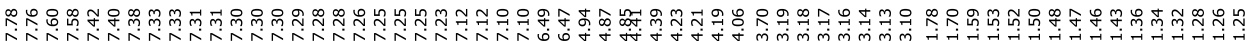

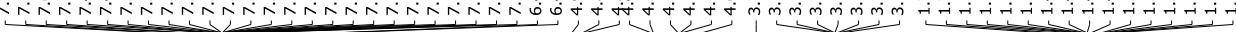
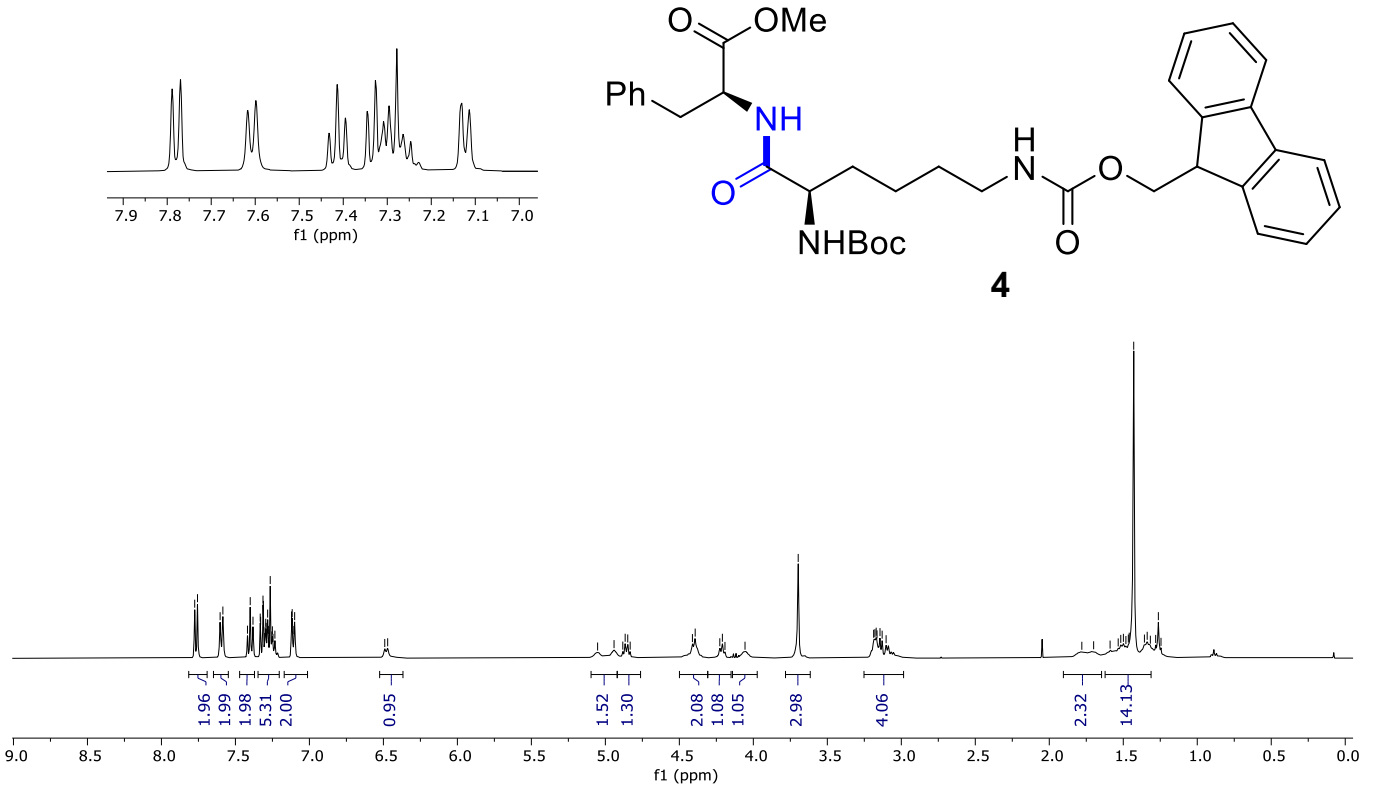

${ }^{1} \mathrm{H}$ NMR Spectrum of 4 in $\mathrm{CDCl}_{3}(400 \mathrm{MHz})$ 


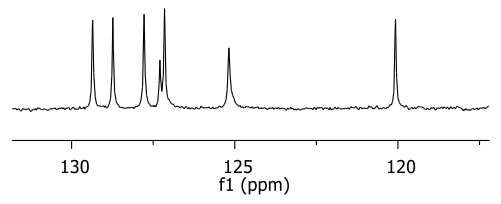<smiles>COC(=O)N[C@@H](CCCCNC(=O)OCC1c2ccccc2-c2ccccc21)C(=O)N[C@@H](Cc1ccccc1)C(=O)OCc1ccccc1</smiles>

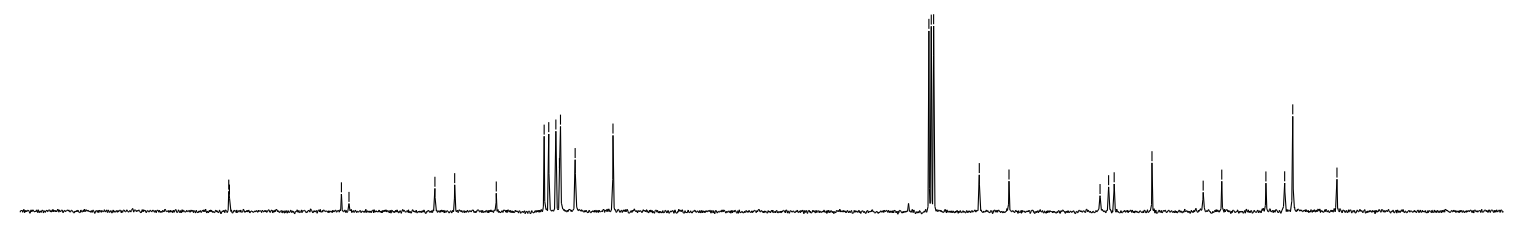

200

${ }^{13} \mathrm{C}$ NMR Spectrum of 4 in $\mathrm{CDCl}_{3}(100 \mathrm{MHz})$

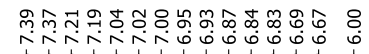

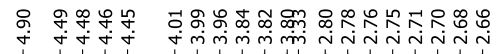<smiles>COC(=O)[C@H](Cc1ccccc1)NC(=O)[C@H](C)NC(=O)OCC1c2ccccc2-c2ccccc21</smiles>

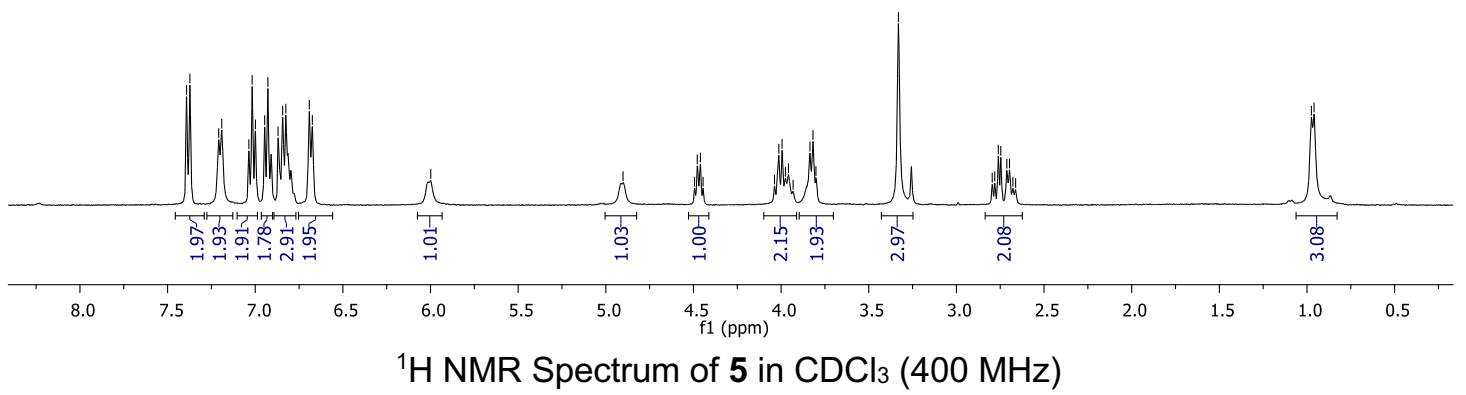



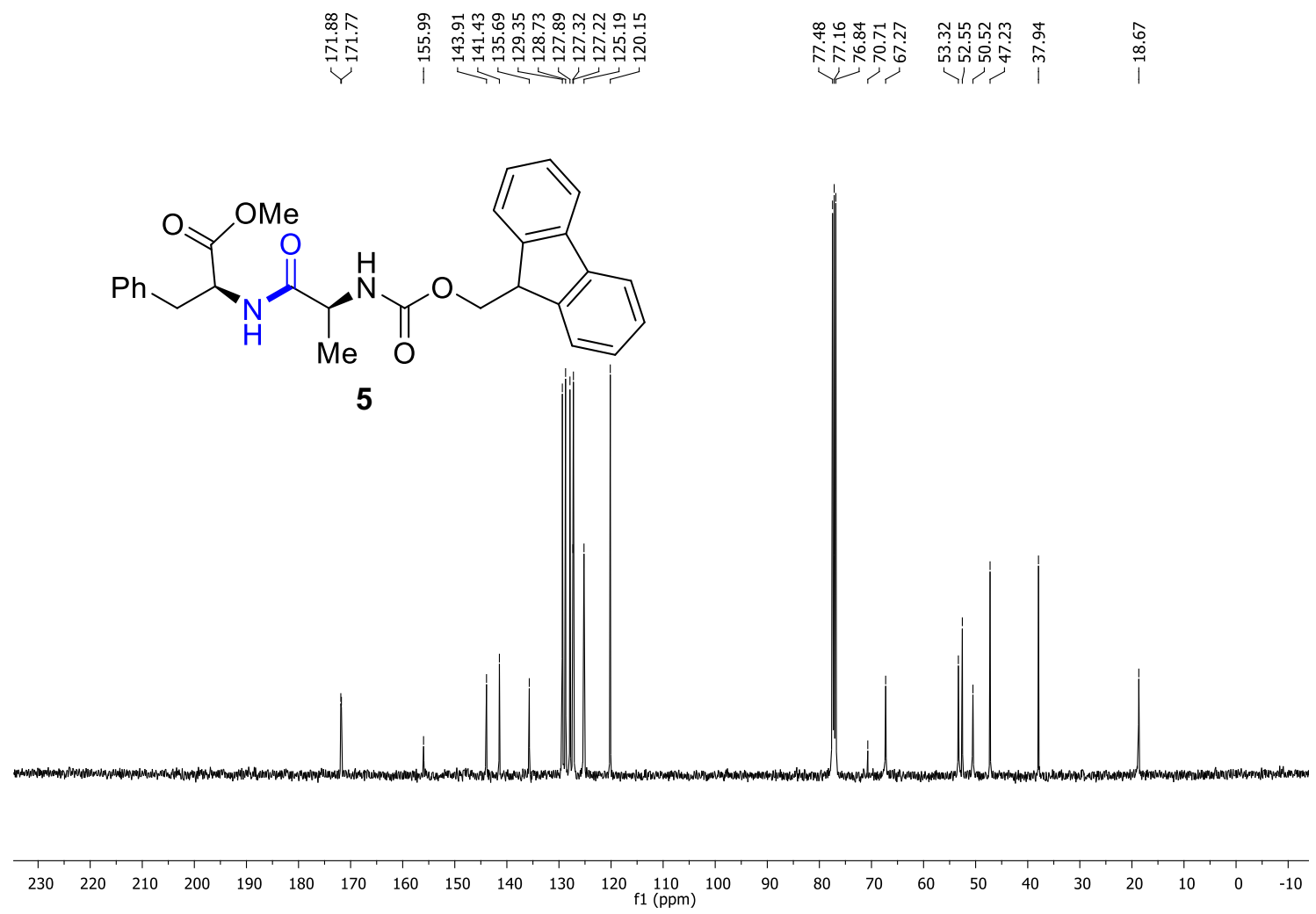

${ }^{13} \mathrm{C}$ NMR Spectrum of 5 in $\mathrm{CDCl}_{3}(100 \mathrm{MHz})$

$\underbrace{\infty}$

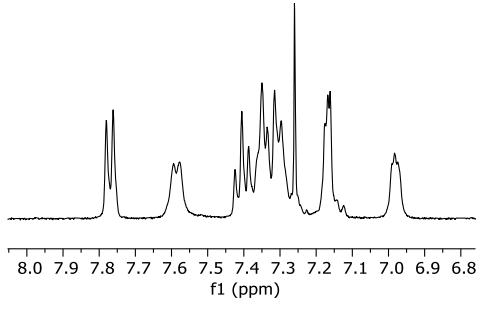<smiles>CC(NC(=O)OCC1c2ccccc2-c2ccccc21)C(=O)N[C@@H](Cc1ccccc1)C(=O)OCc1ccccc1</smiles>

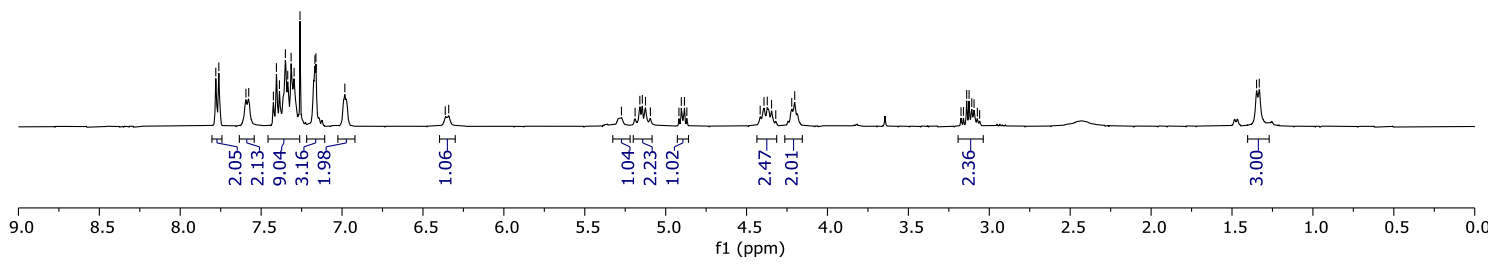

${ }^{1} \mathrm{H}$ NMR Spectrum of 6 in $\mathrm{CDCl}_{3}(400 \mathrm{MHz})$ 

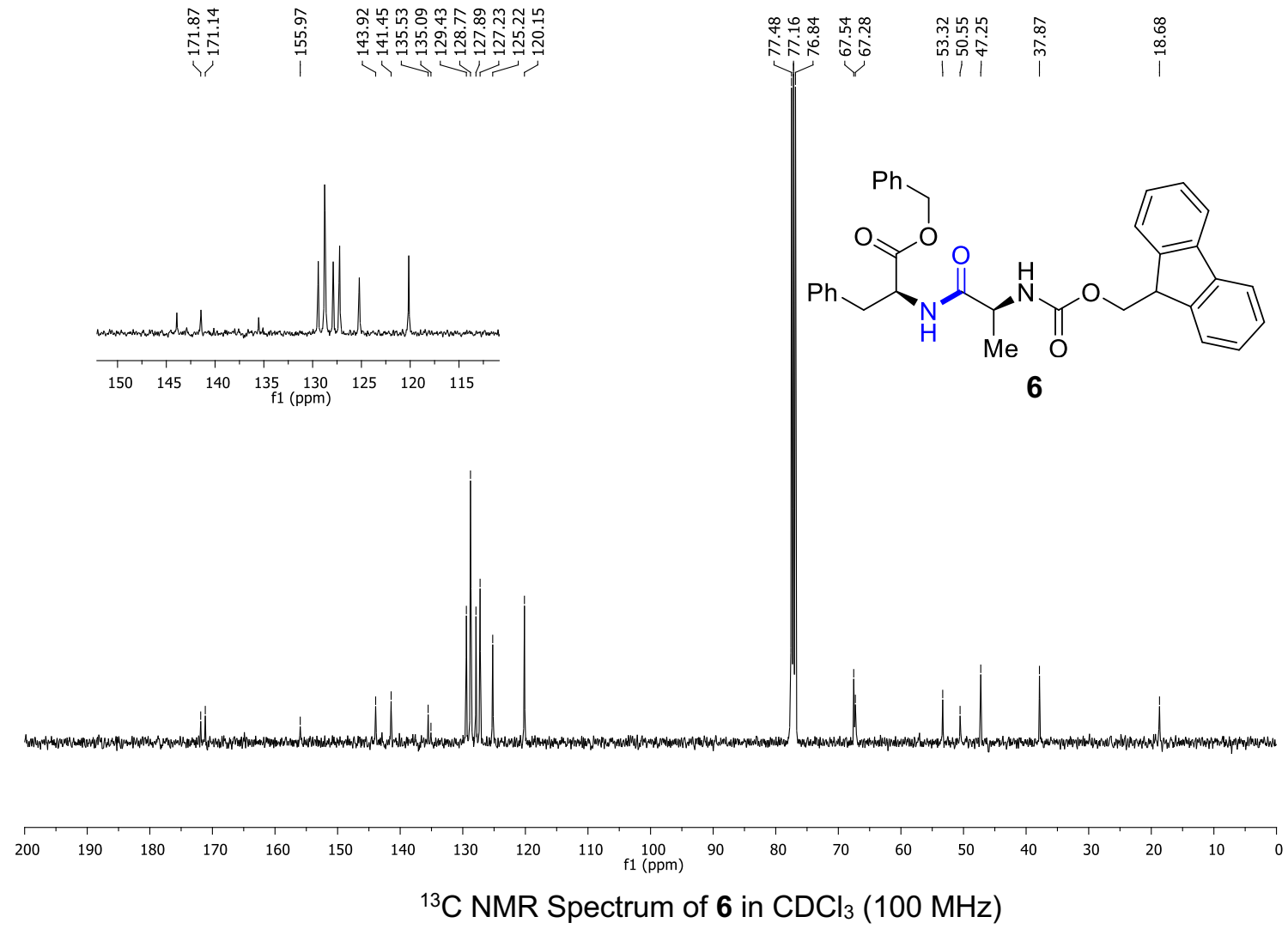

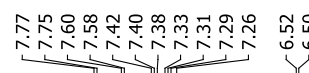
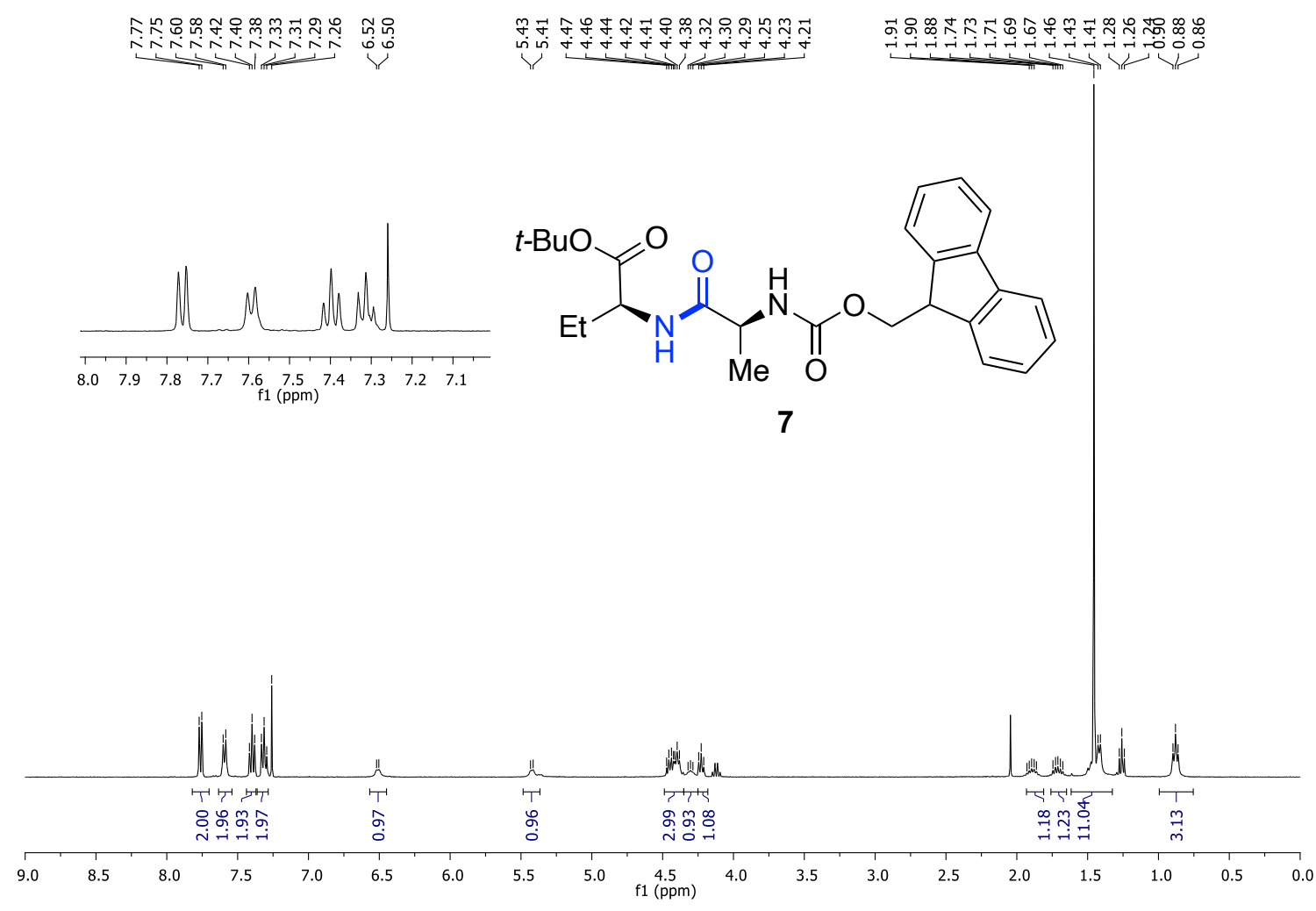

${ }^{1} \mathrm{H}$ NMR Spectrum of 7 in $\mathrm{CDCl}_{3}(400 \mathrm{MHz})$ 


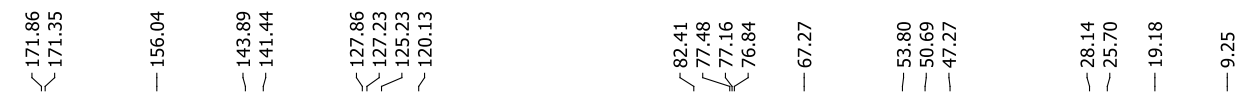<smiles>CCC(C)OC(=O)[C@H](CC)NC(=O)[C@H](C)NC(=O)OCC1c2ccccc2-c2ccccc21</smiles>
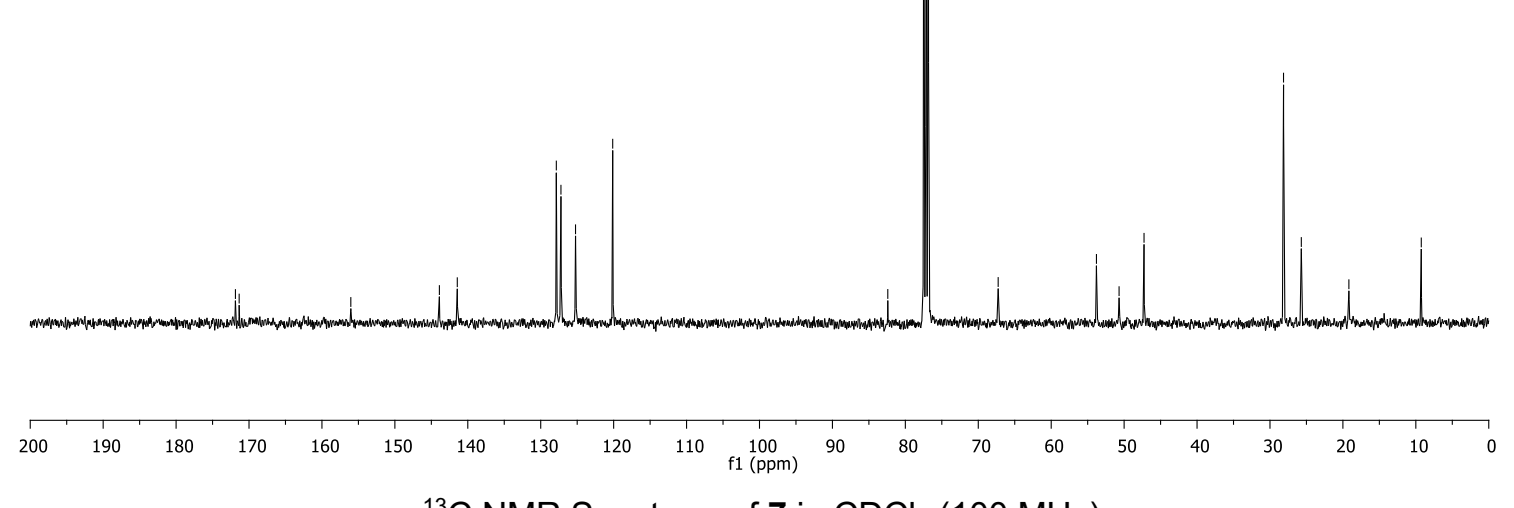

${ }^{13} \mathrm{C}$ NMR Spectrum of 7 in $\mathrm{CDCl}_{3}(100 \mathrm{MHz})$
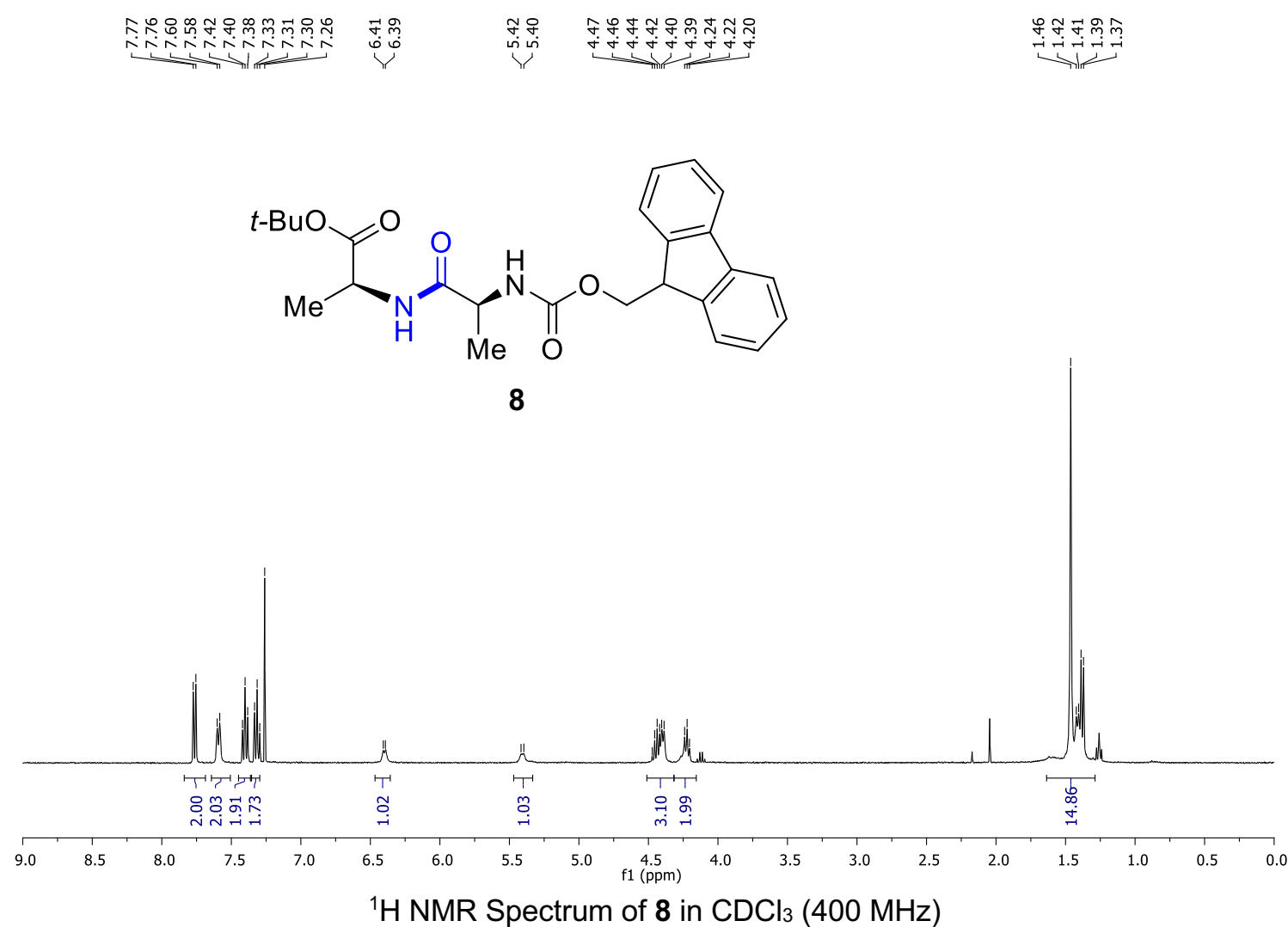

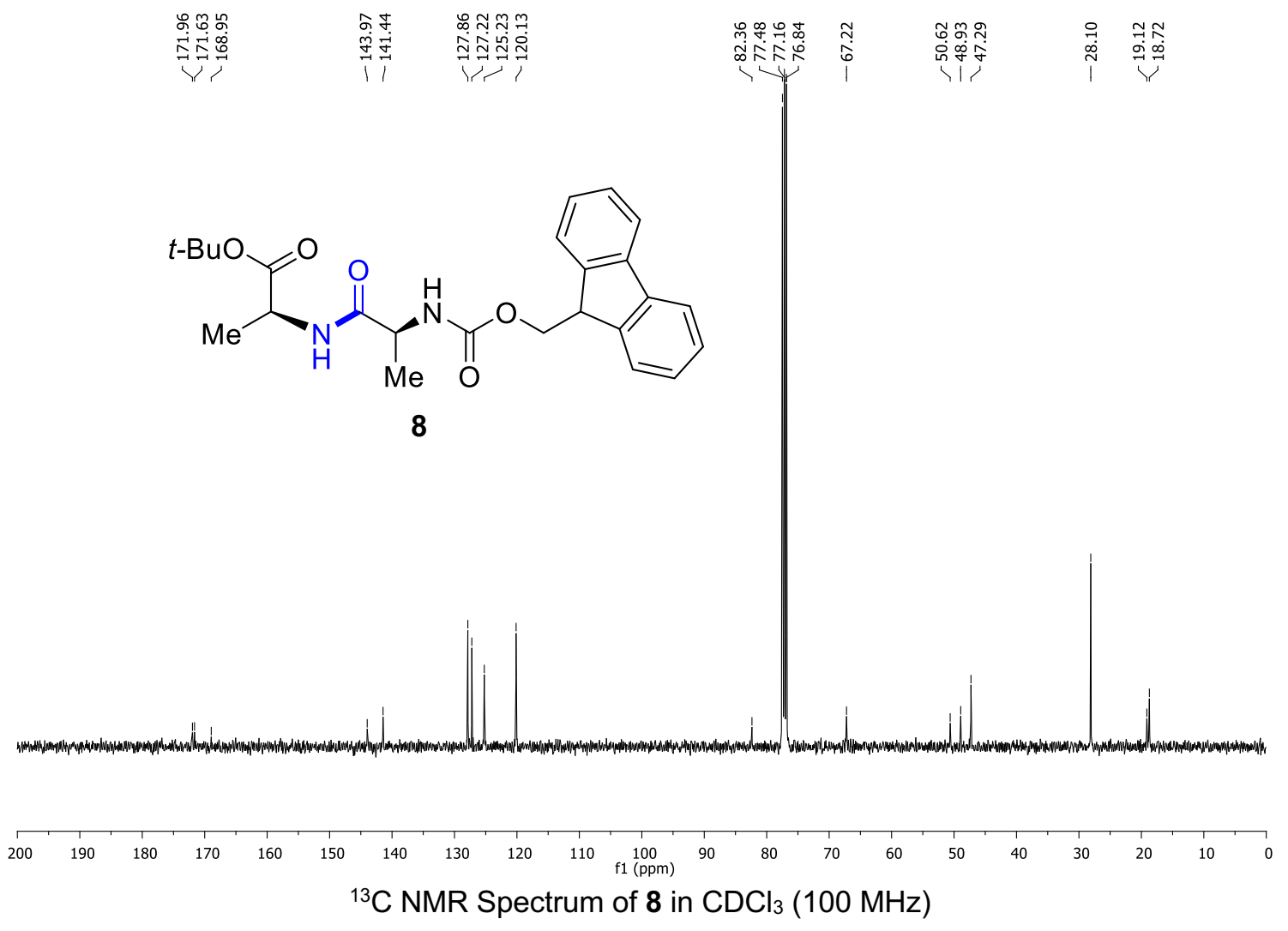

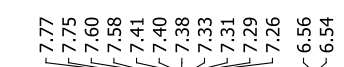
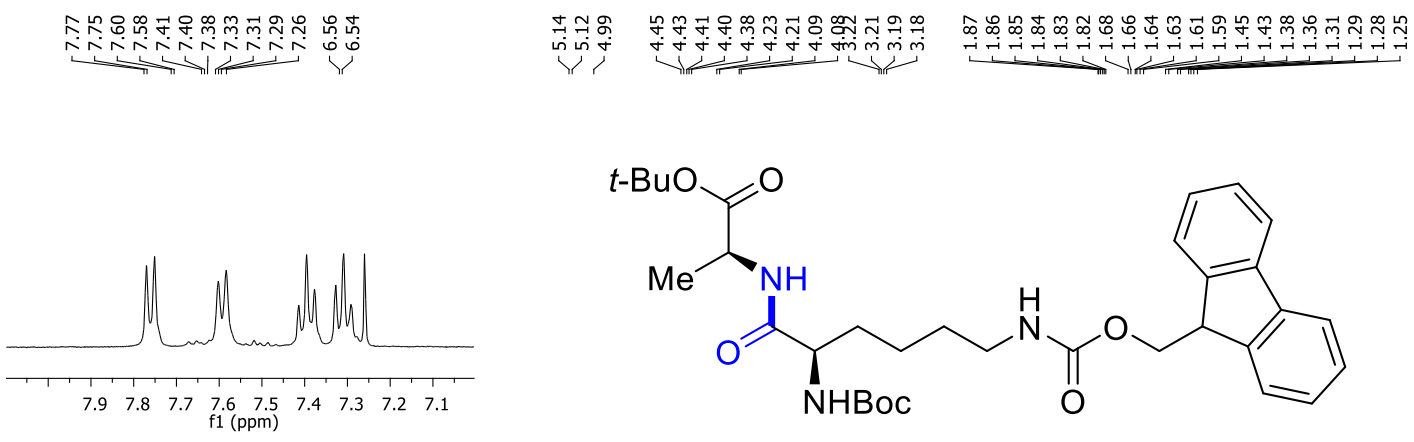

9

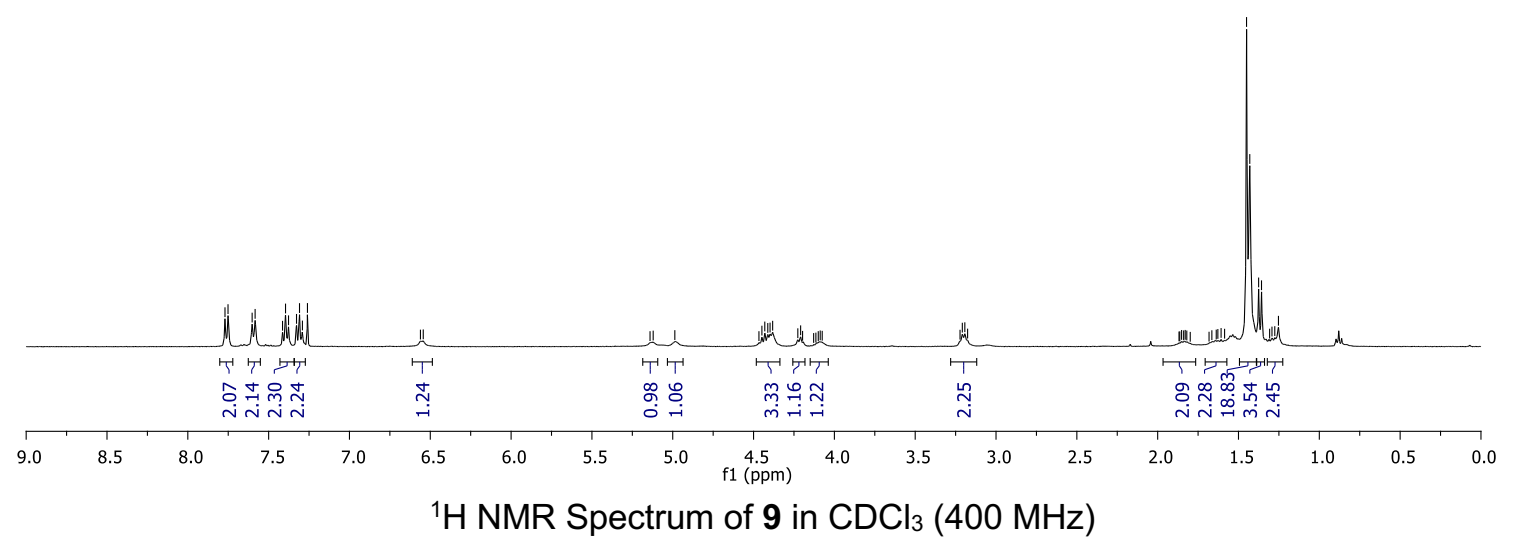



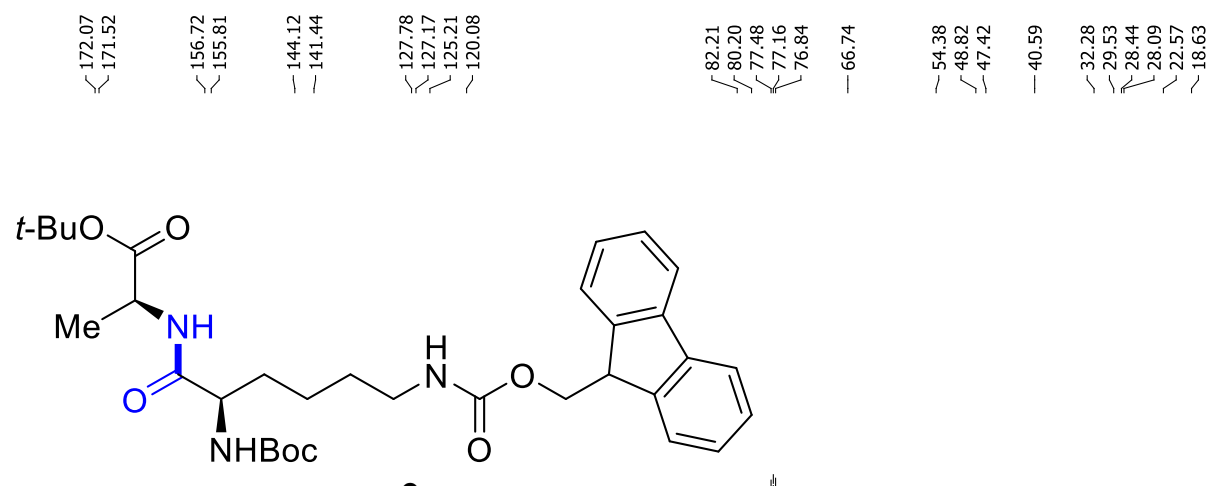

9
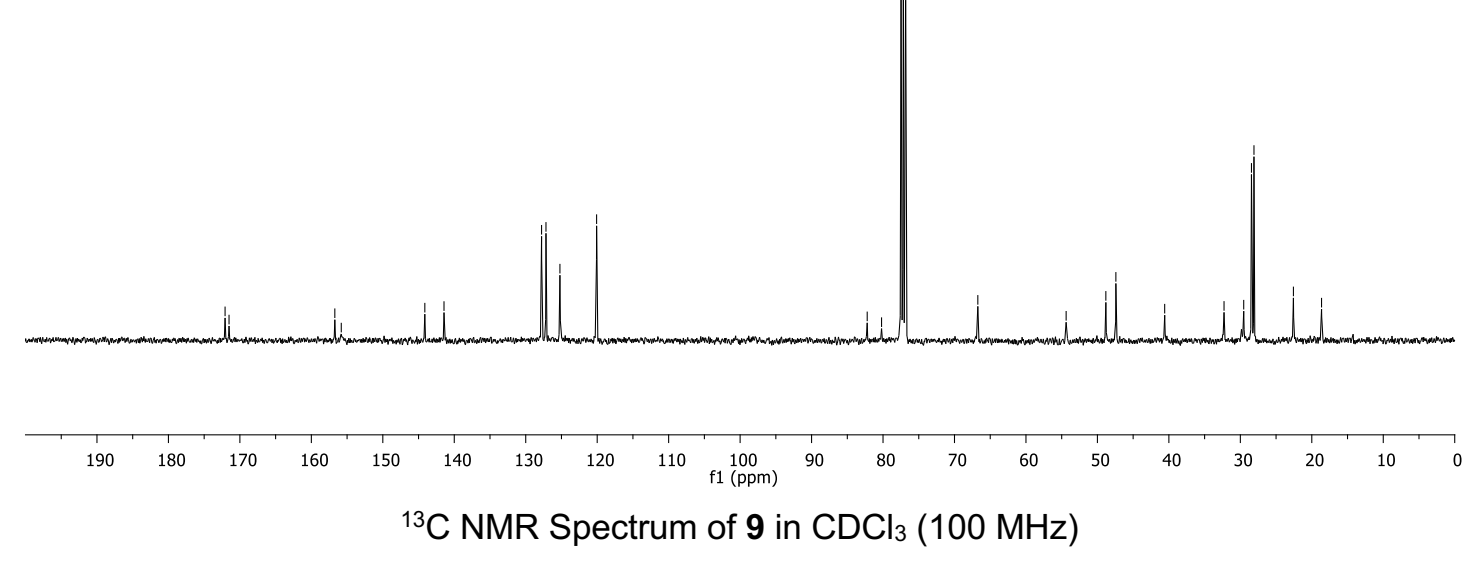

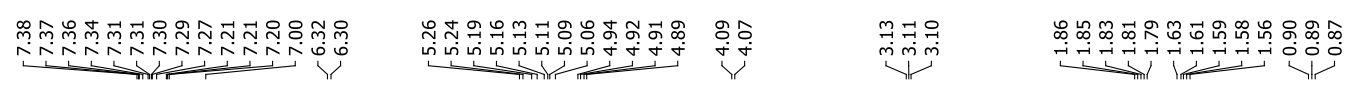<smiles>CC[C@H](NC(=O)OCc1ccccc1)C(=O)N[C@@H](Cc1ccccc1)C(=O)OCc1ccccc1</smiles>

10

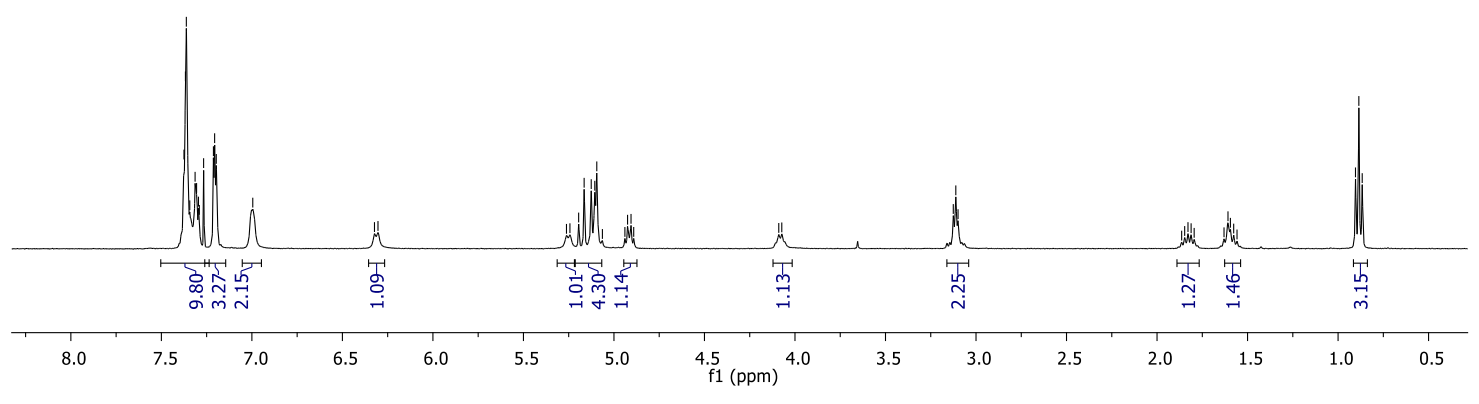

${ }^{1} \mathrm{H}$ NMR Spectrum of 10 in $\mathrm{CDCl}_{3}(400 \mathrm{MHz})$ 


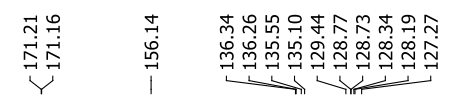

棺尔迸
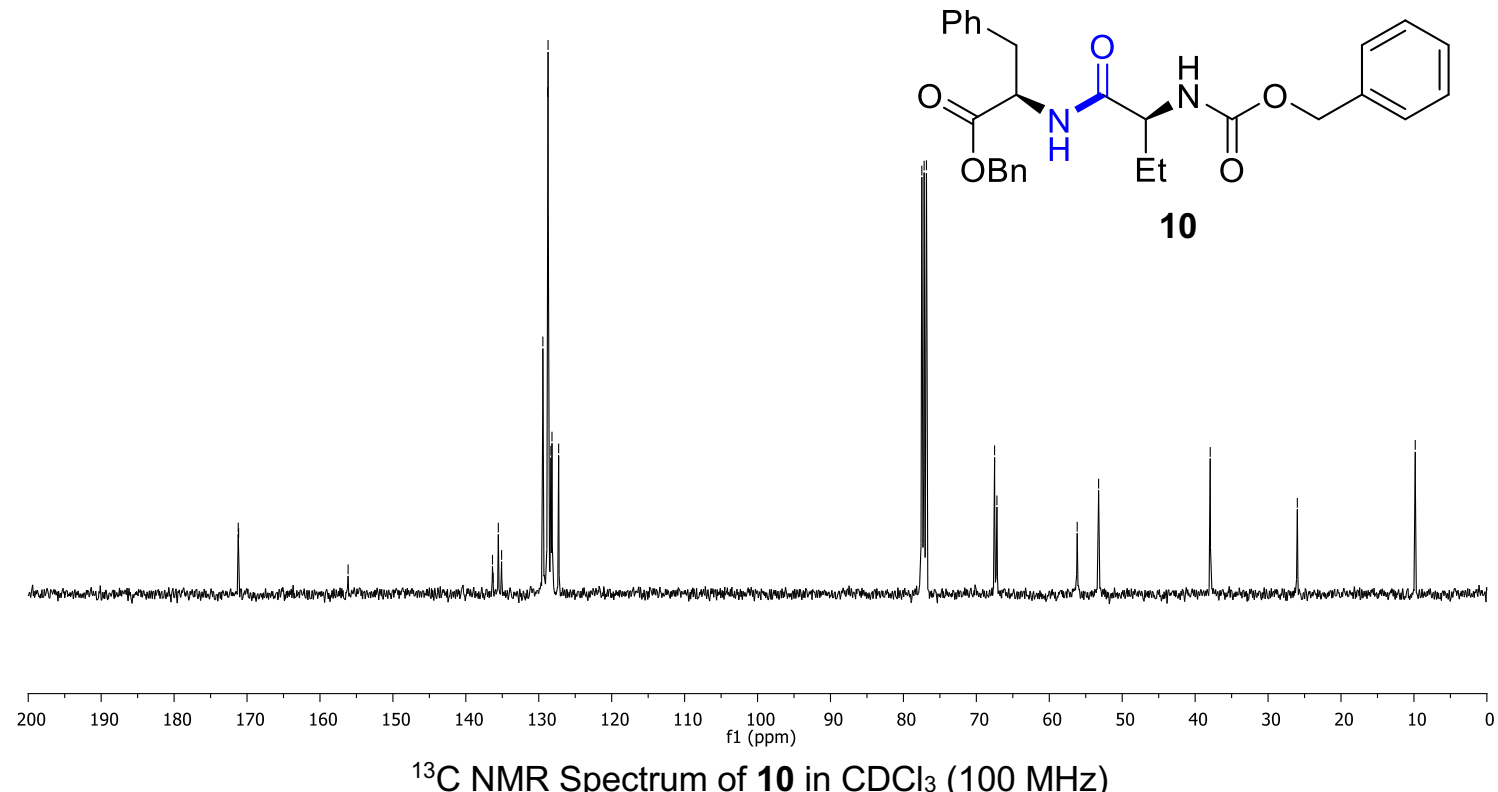

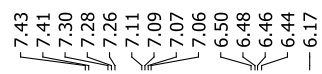

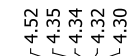

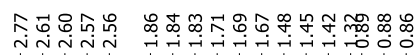

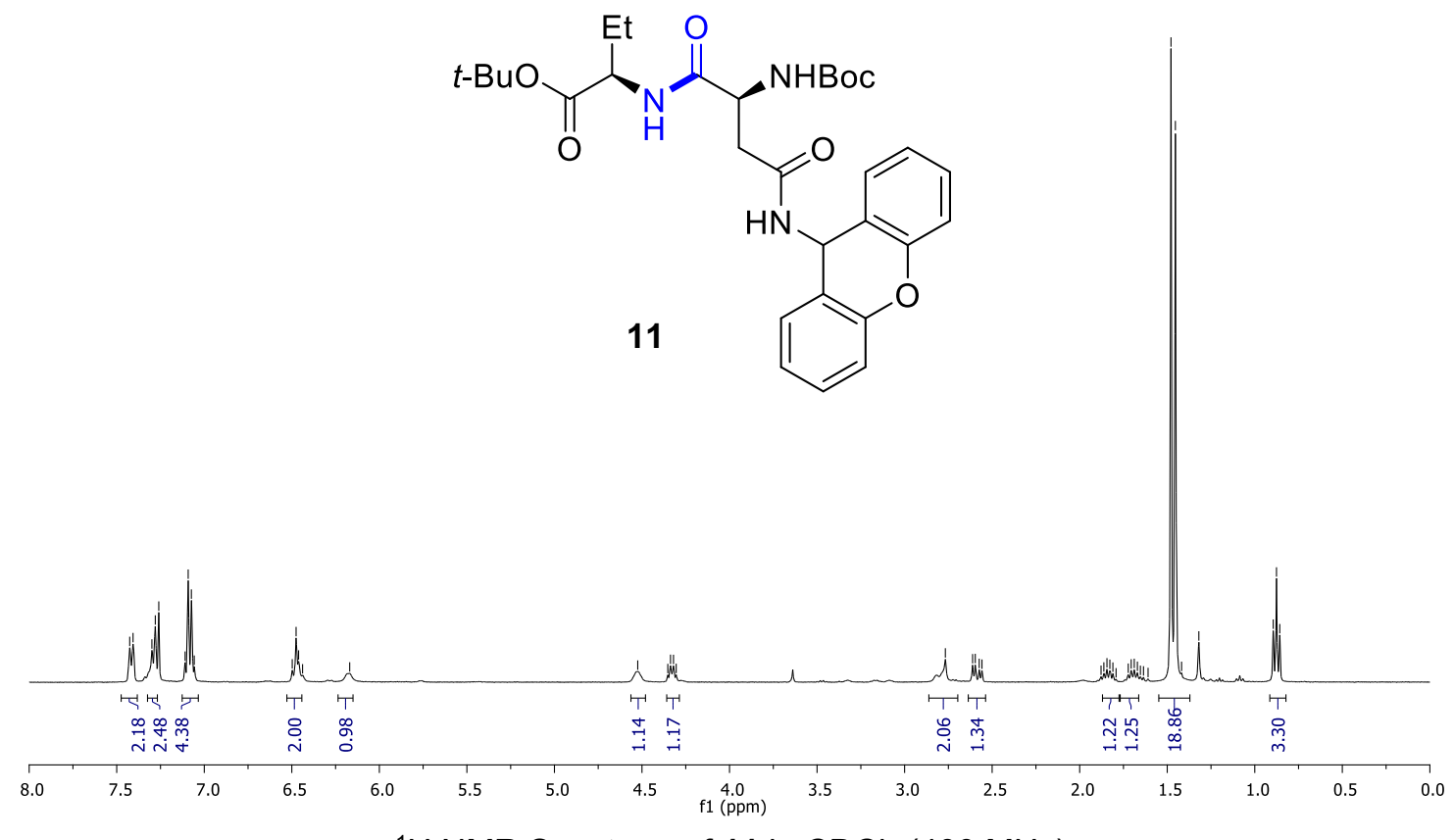

${ }^{1} \mathrm{H}$ NMR Spectrum of 11 in $\mathrm{CDCl}_{3}(400 \mathrm{MHz})$ 

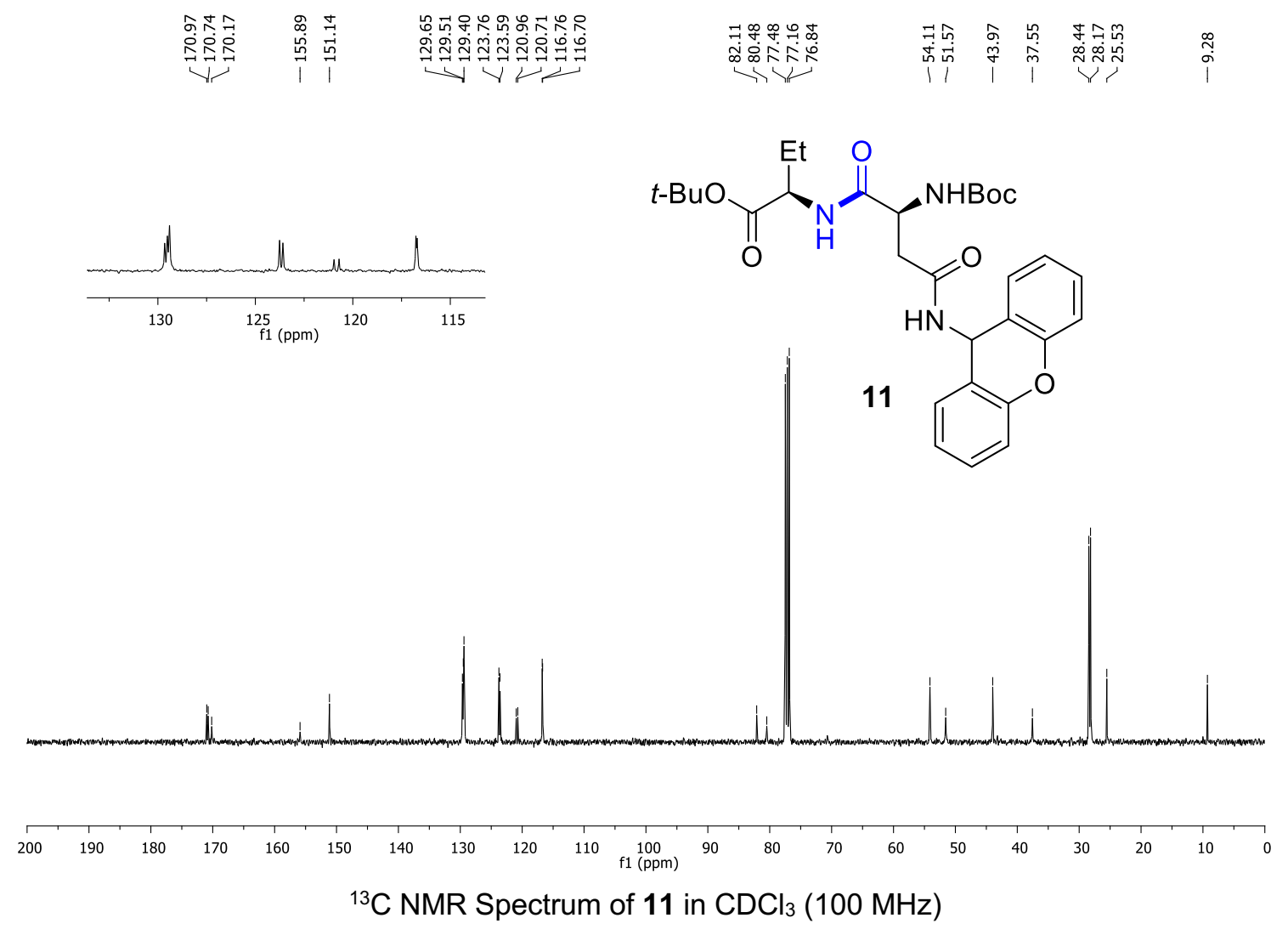

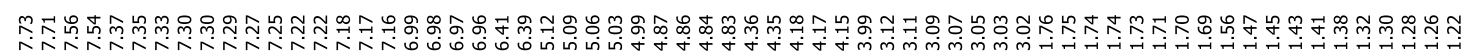

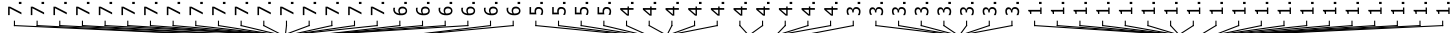

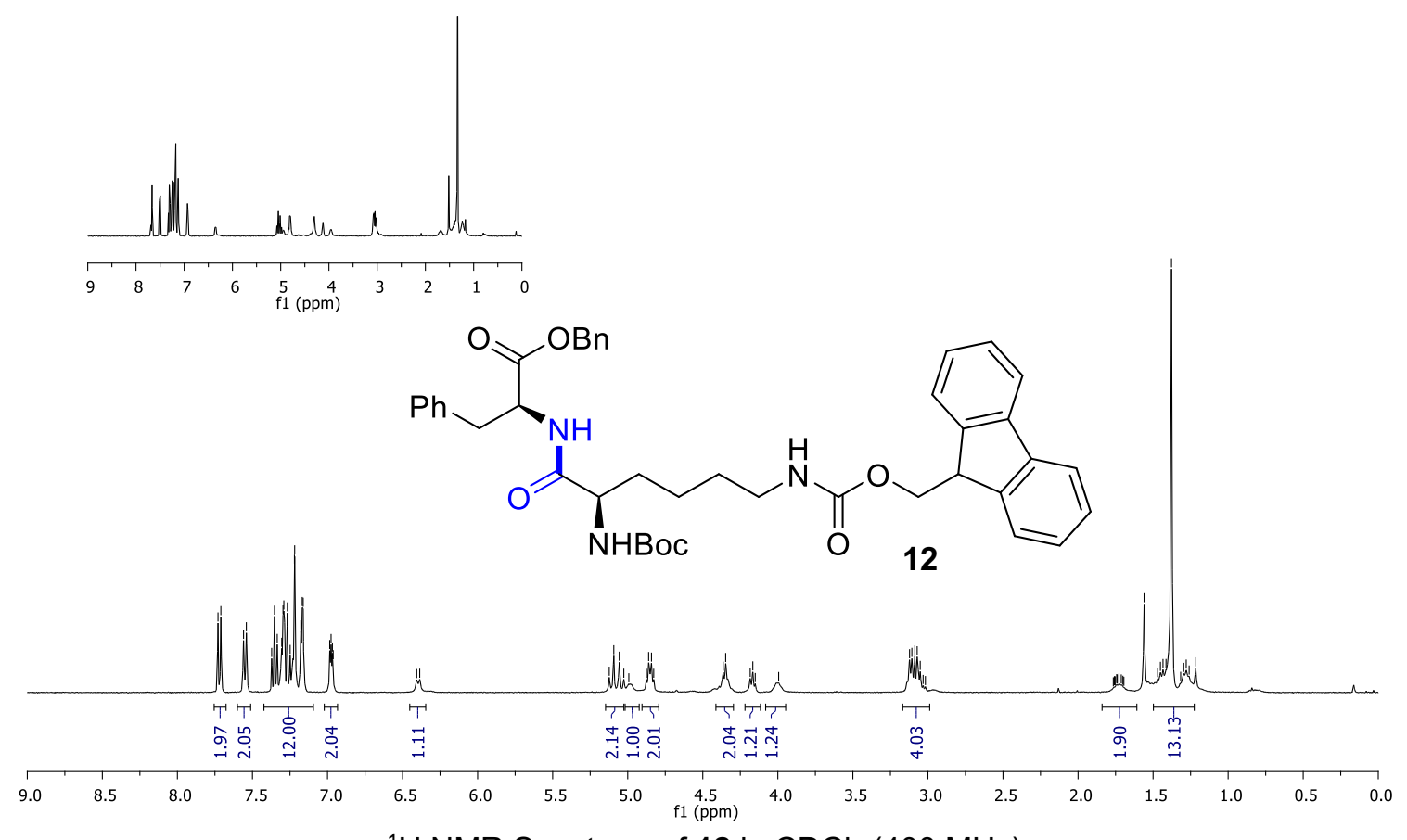

${ }^{1} \mathrm{H}$ NMR Spectrum of 12 in $\mathrm{CDCl}_{3}(400 \mathrm{MHz})$ 

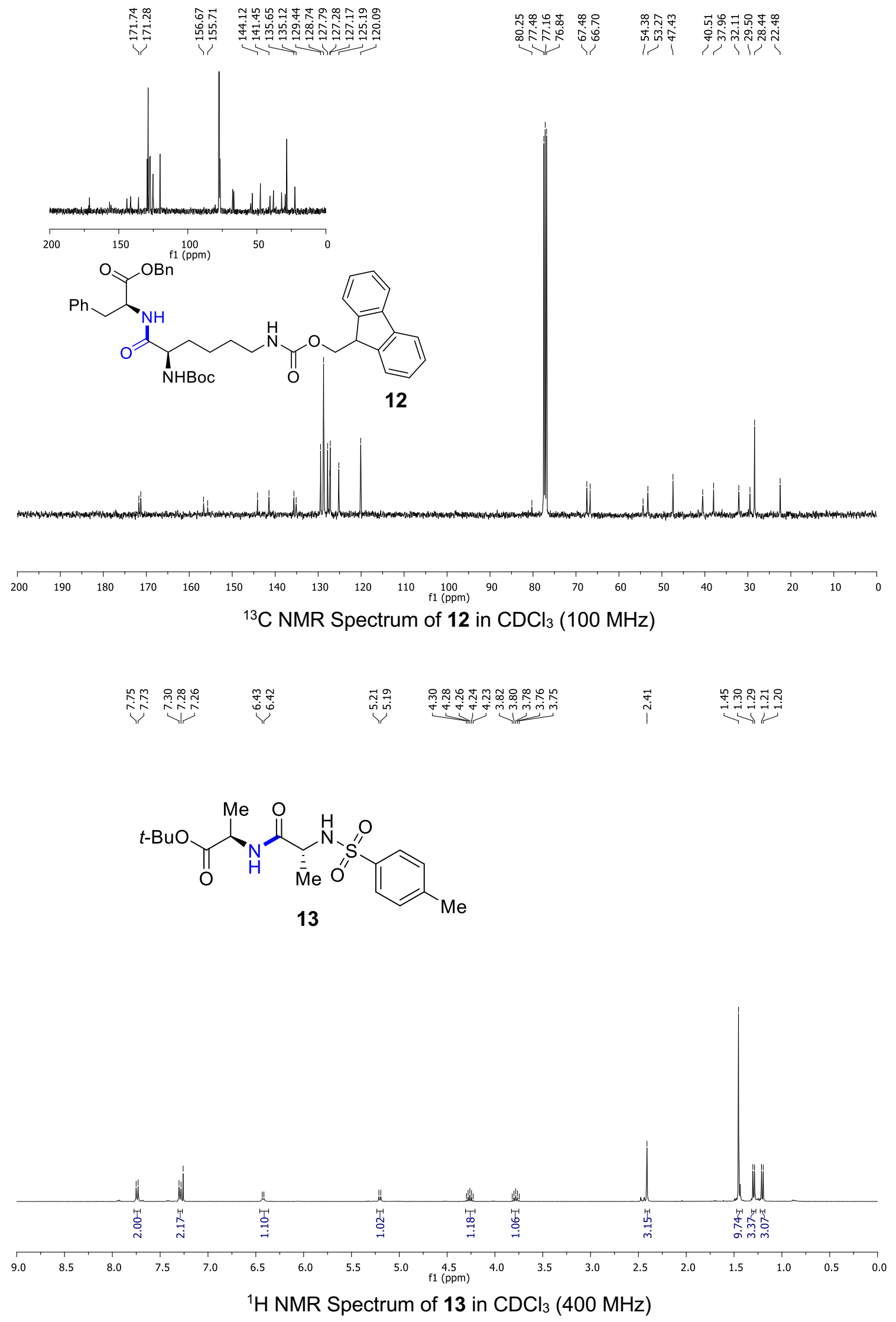


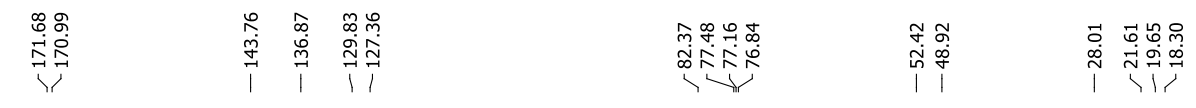<smiles>Cc1ccc(S(=O)(=O)NC(C)C(=O)NC(C)C(=O)OC(C)(C)C)cc1</smiles>
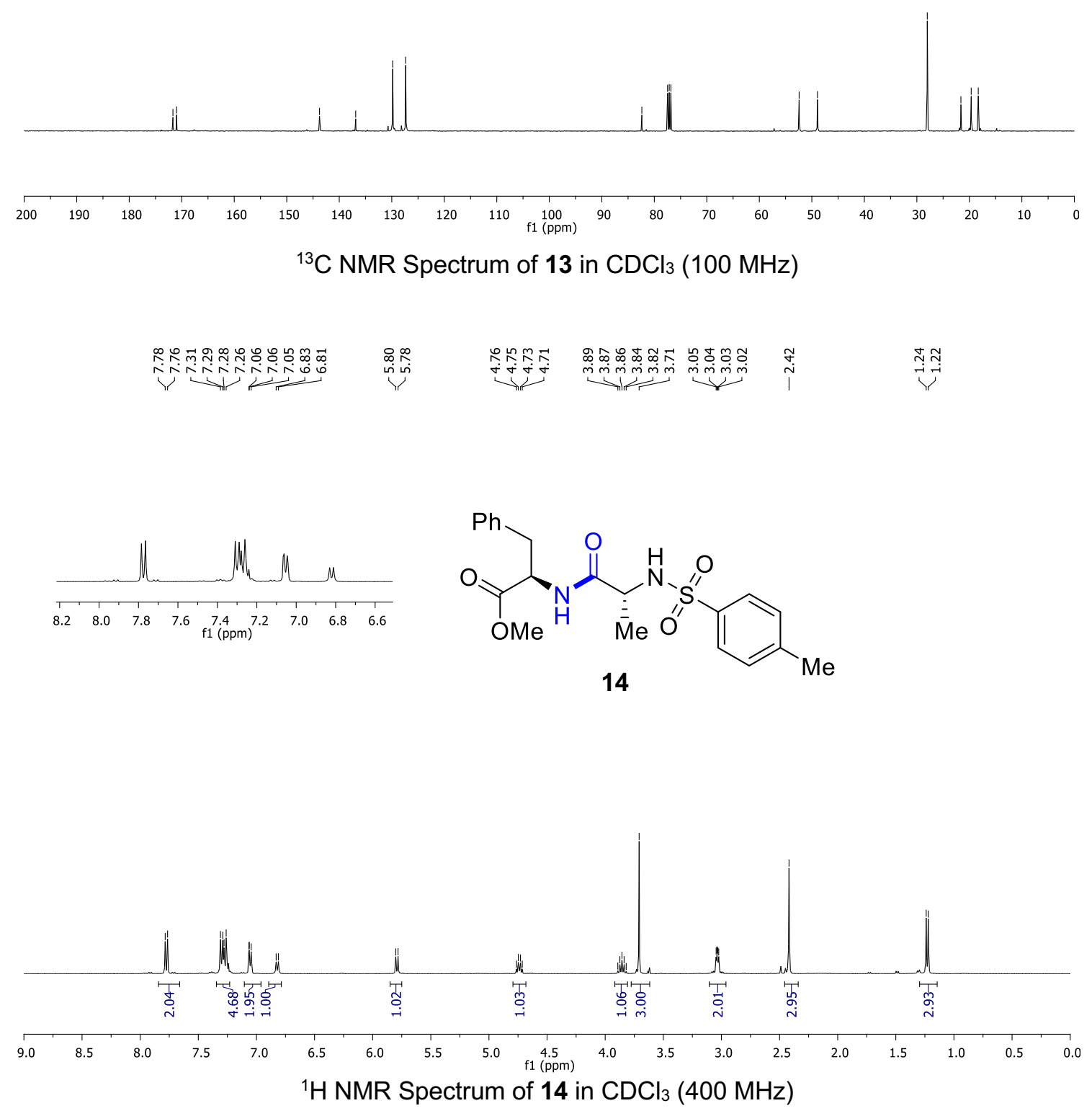

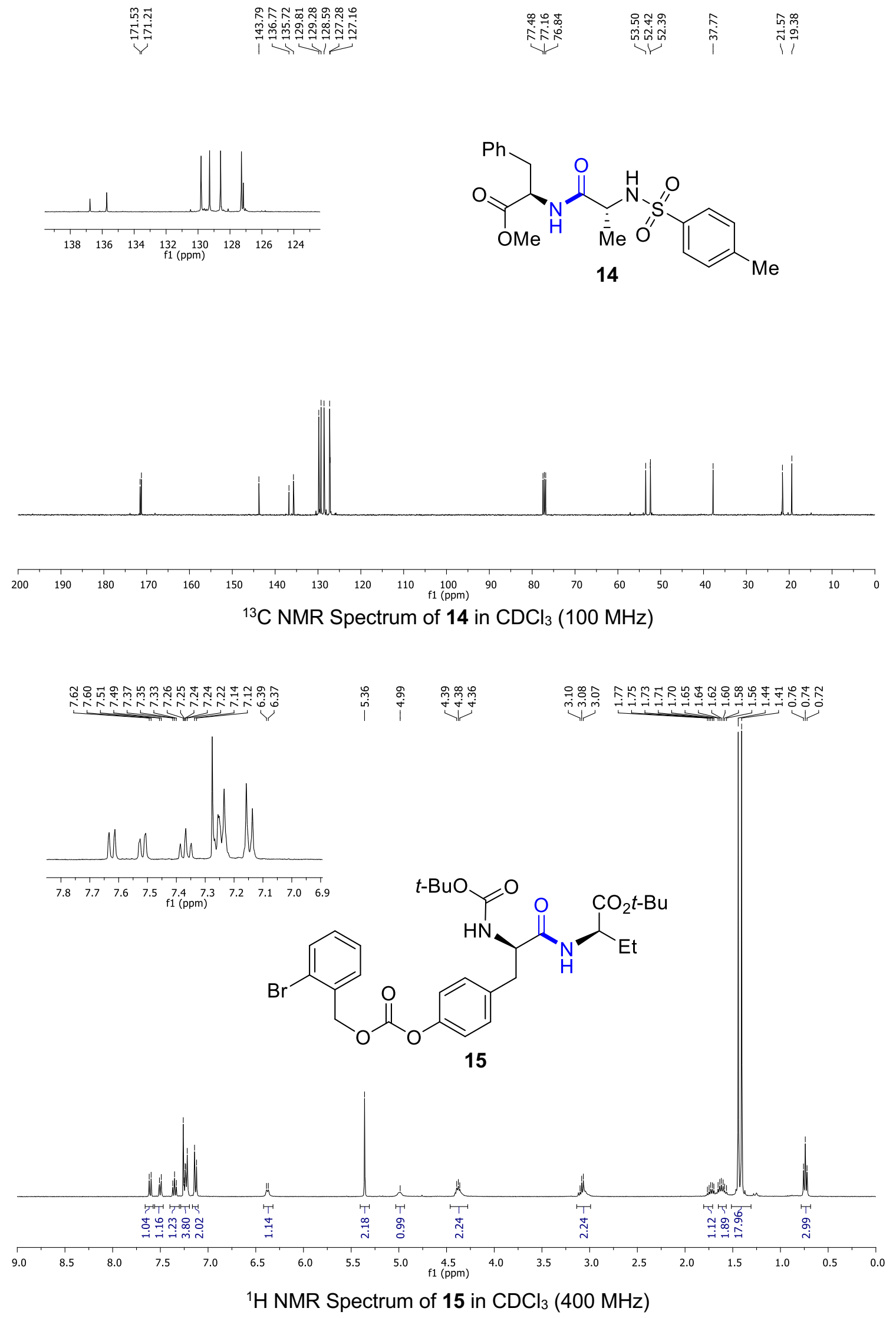

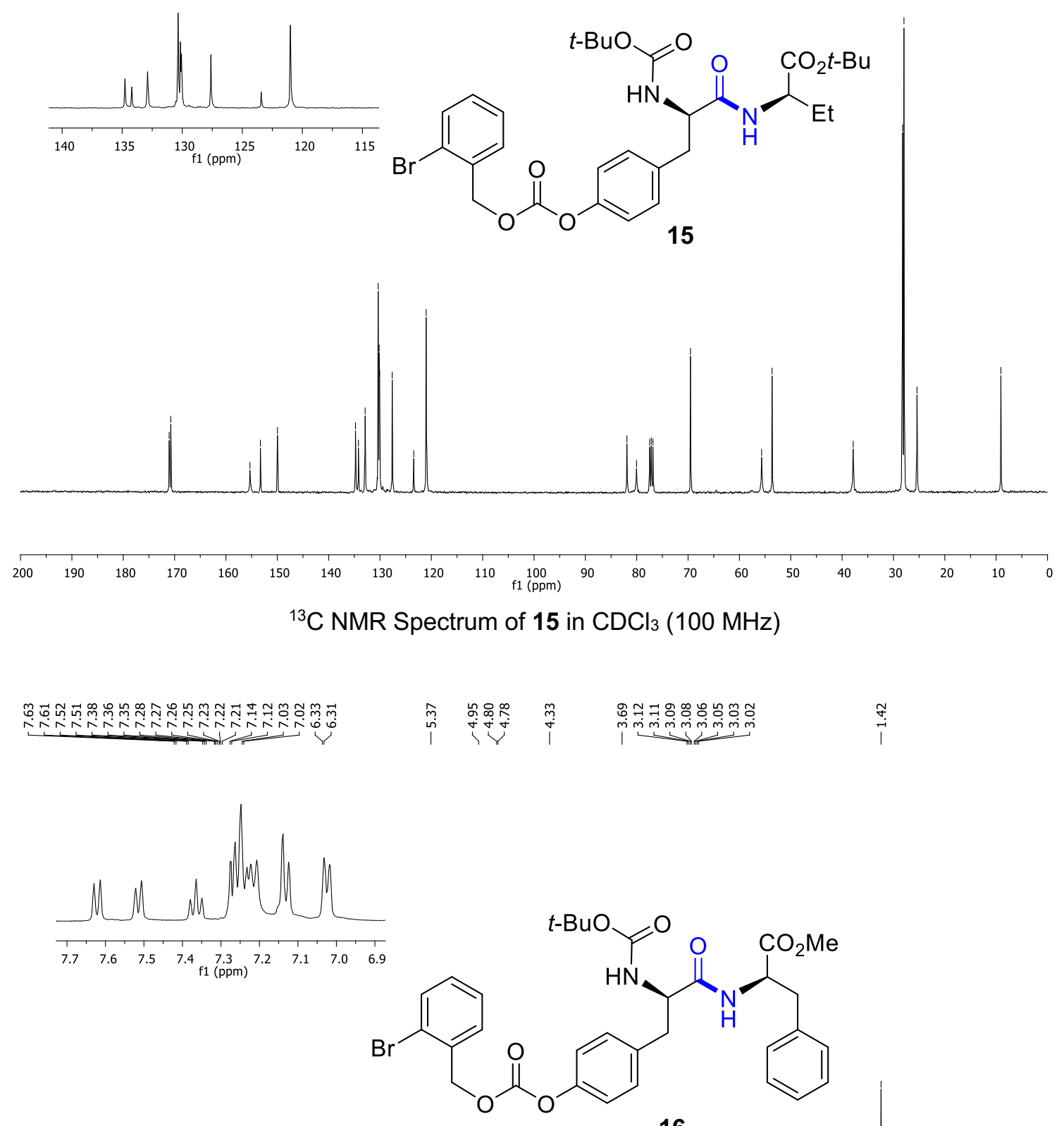

16

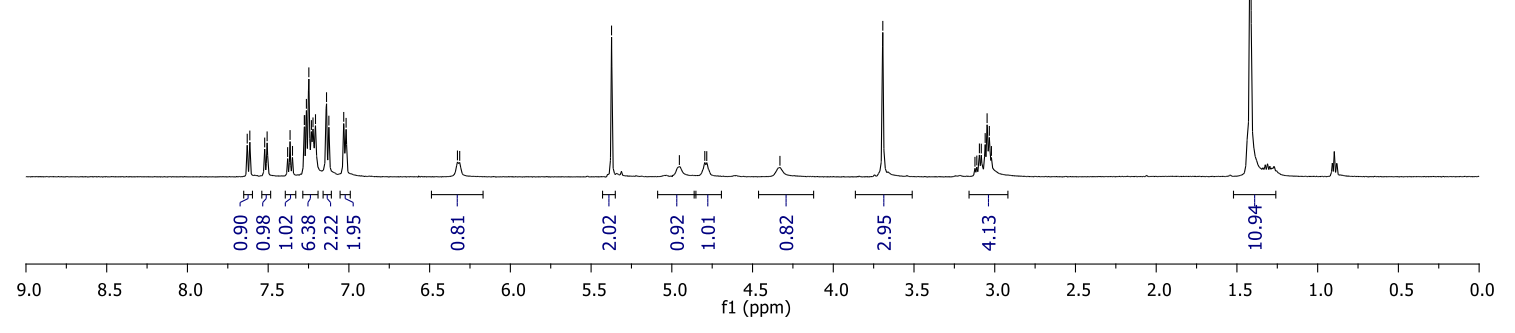

${ }^{1} \mathrm{H}$ NMR Spectrum of 16 in $\mathrm{CDCl}_{3}(500 \mathrm{MHz})$ 


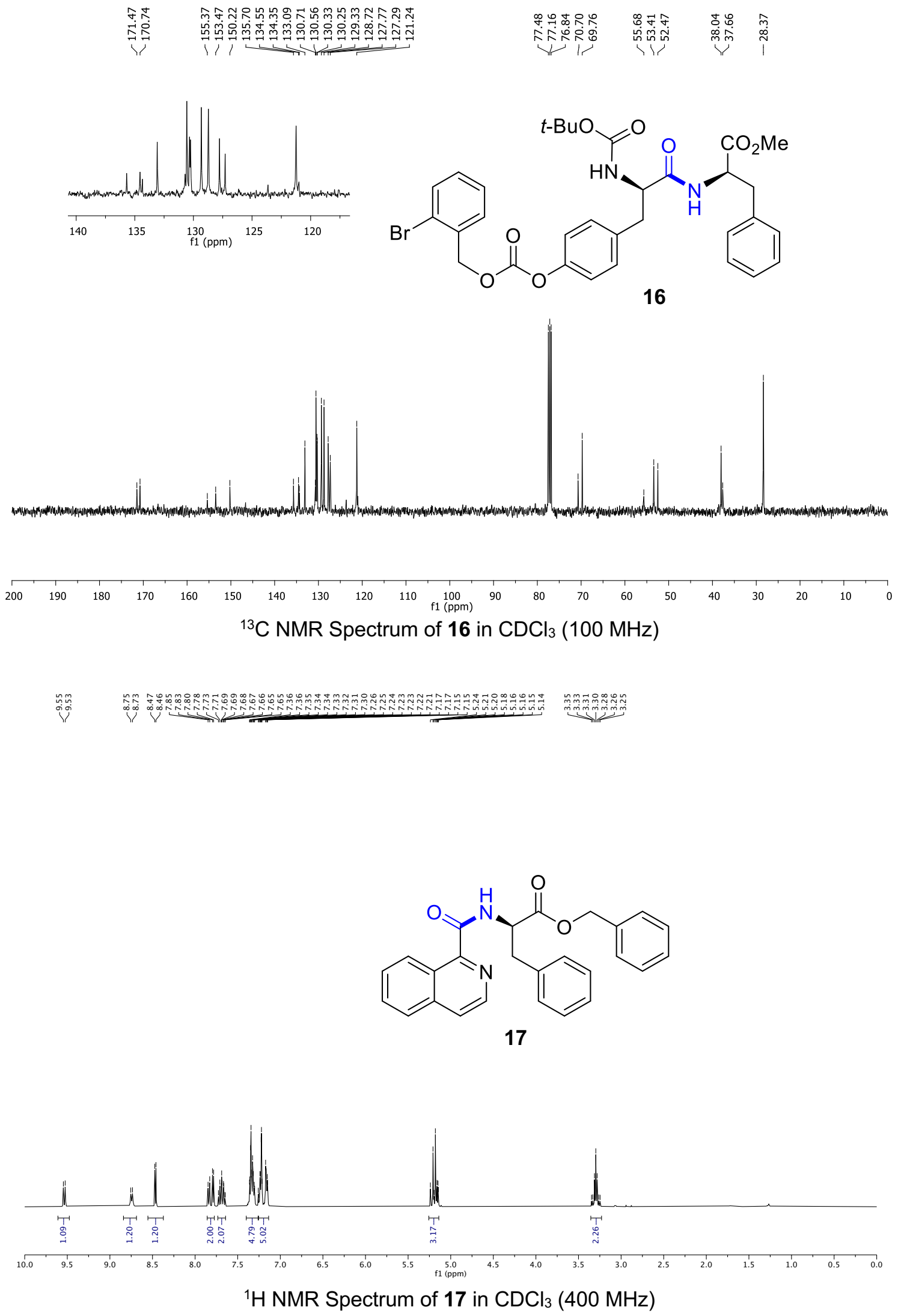



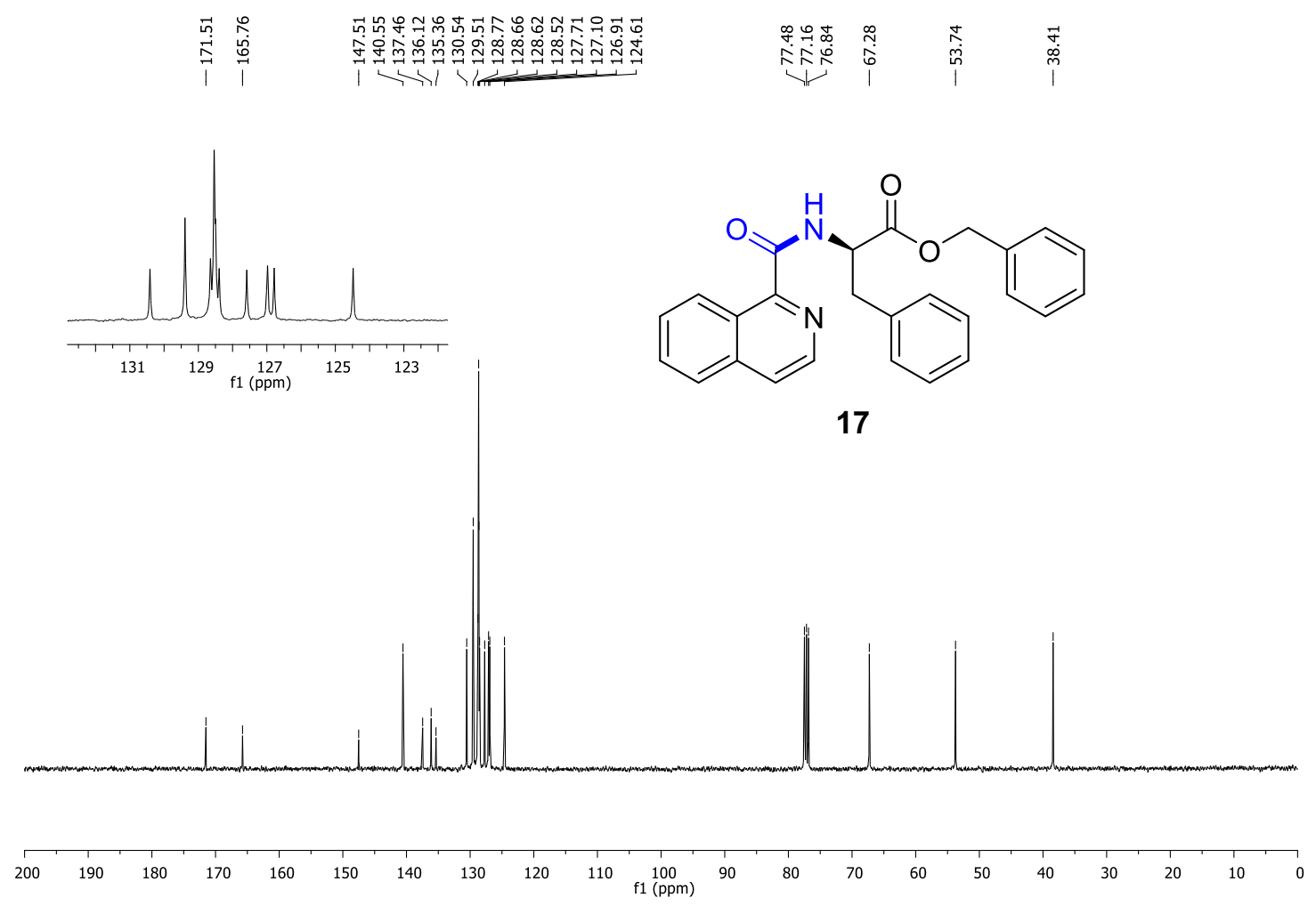

${ }^{13} \mathrm{C}$ NMR Spectrum of 17 in $\mathrm{CDCl}_{3}(100 \mathrm{MHz})$

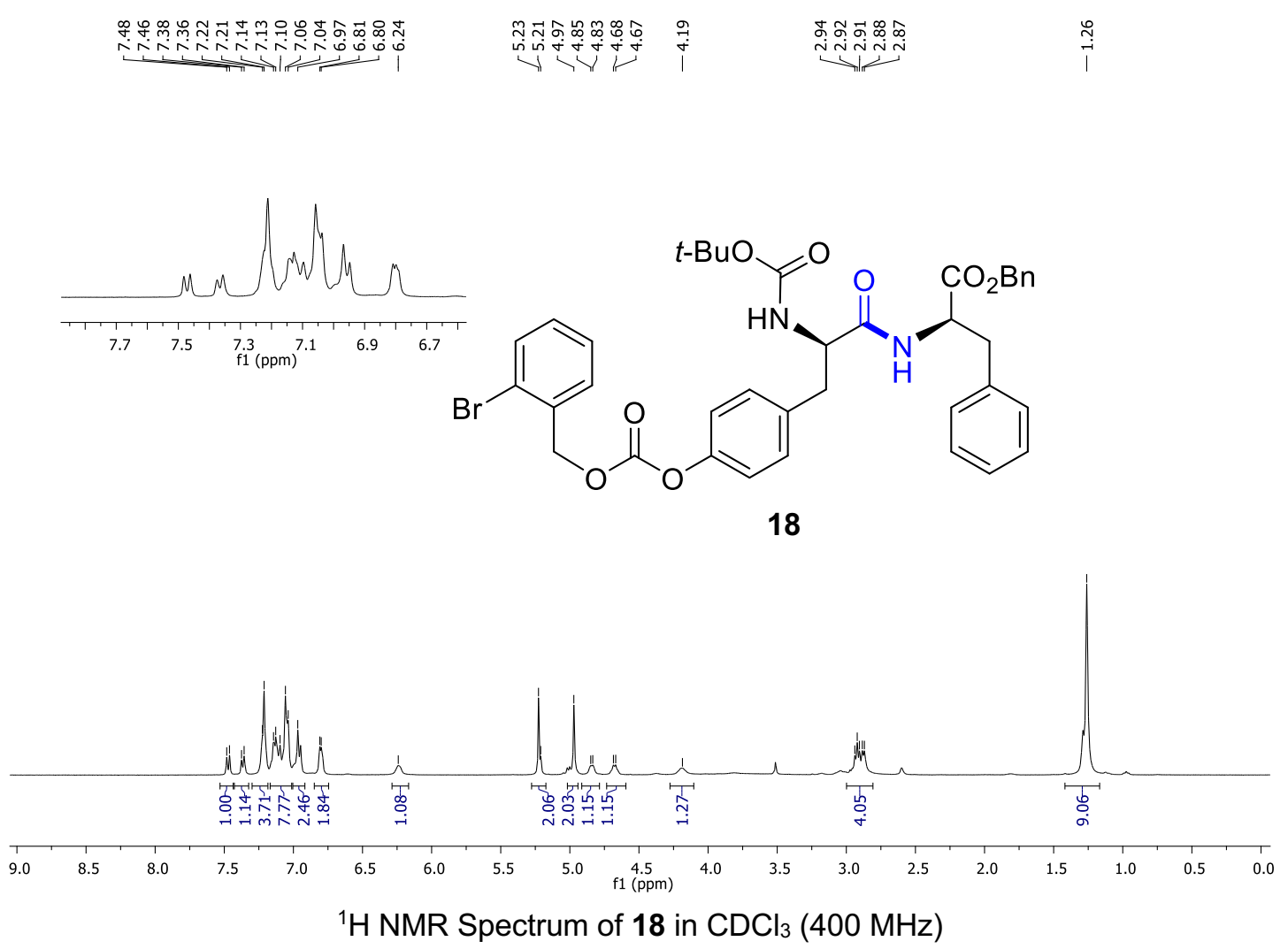



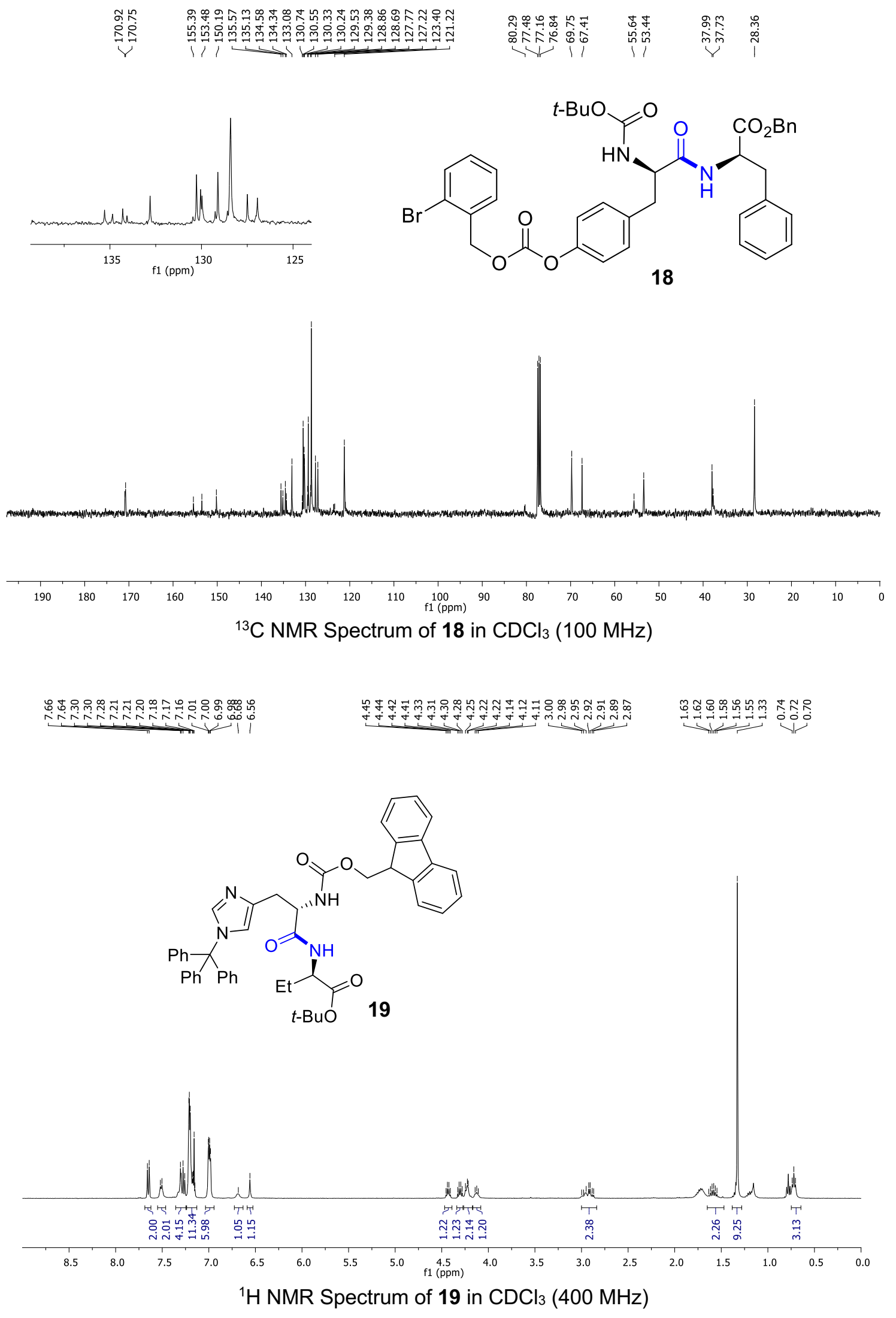

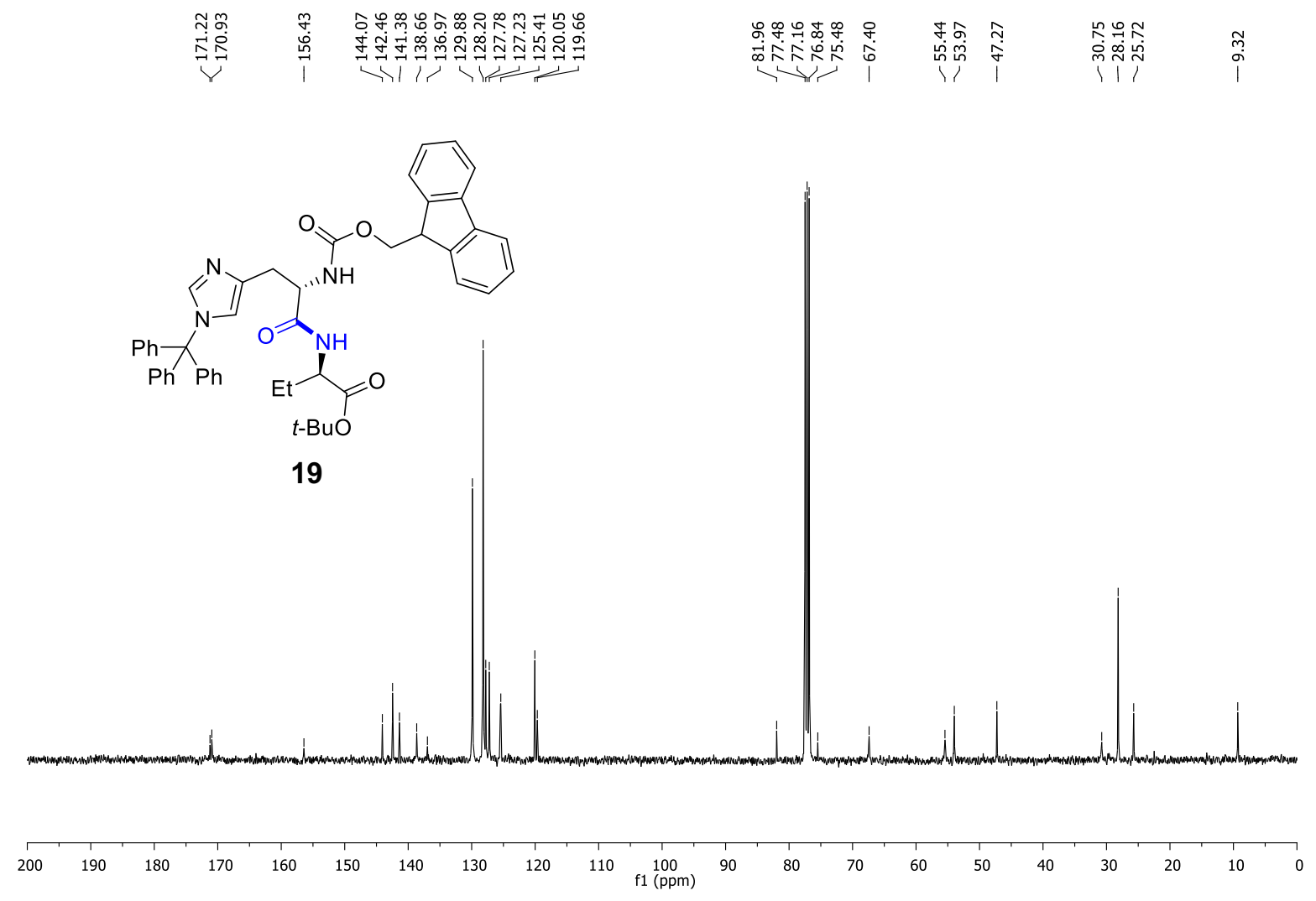

${ }^{13} \mathrm{C}$ NMR Spectrum of 19 in $\mathrm{CDCl}_{3}(100 \mathrm{MHz})$
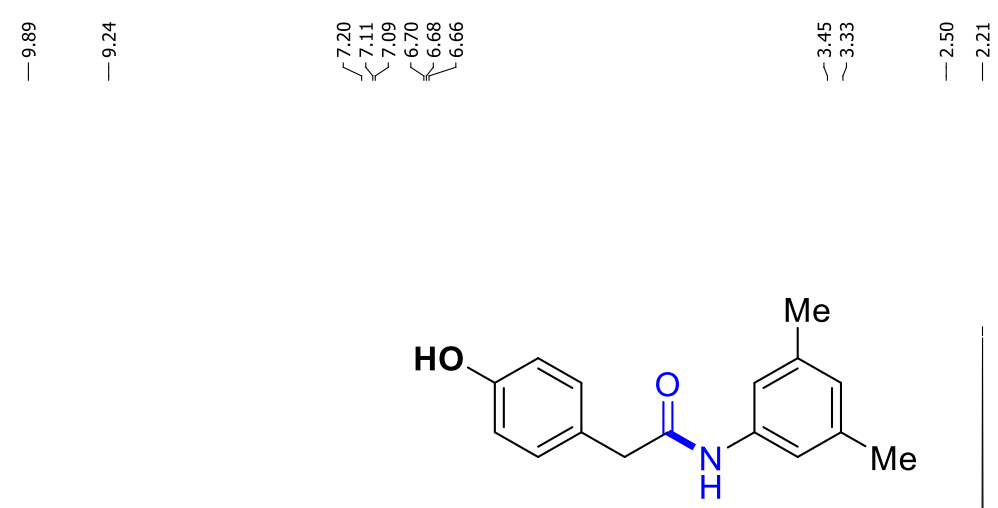

20

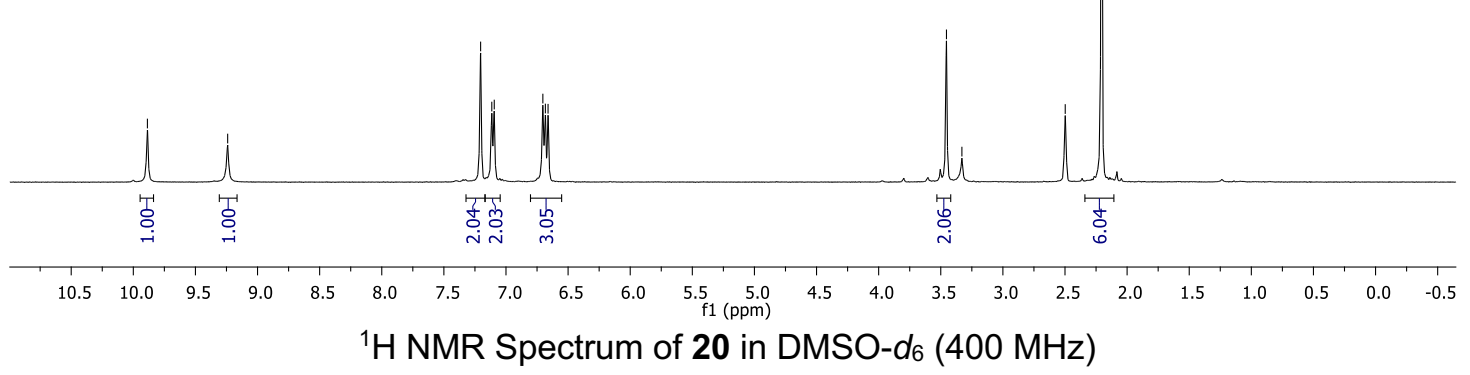



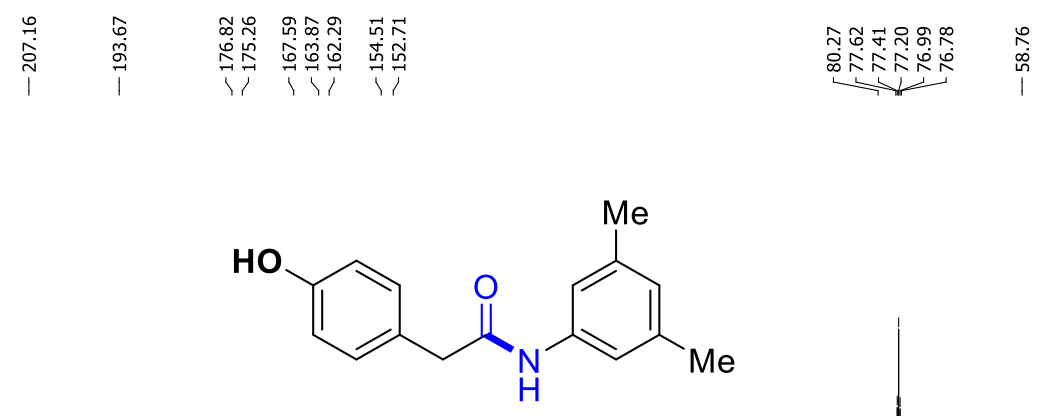

20
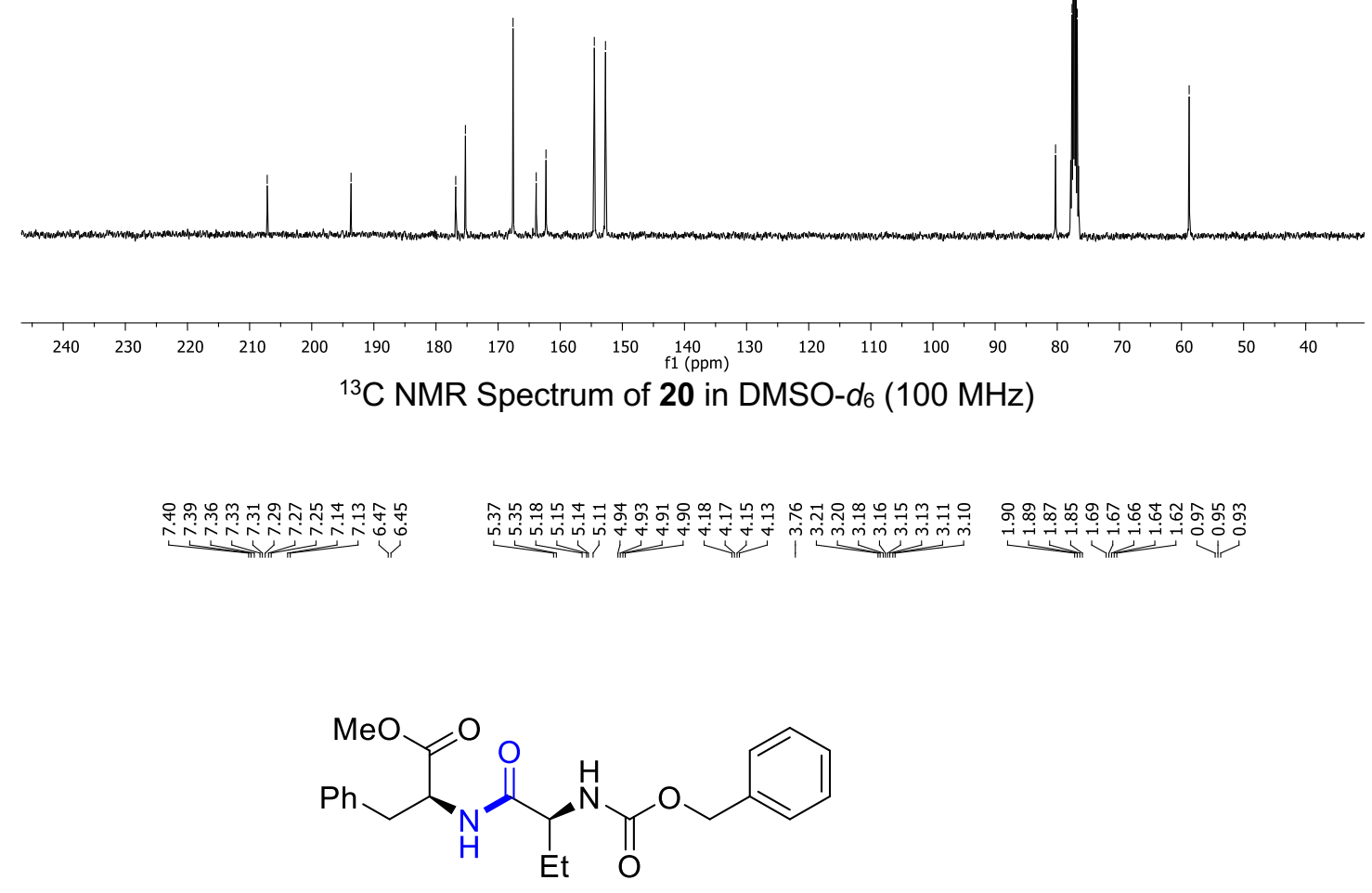

21

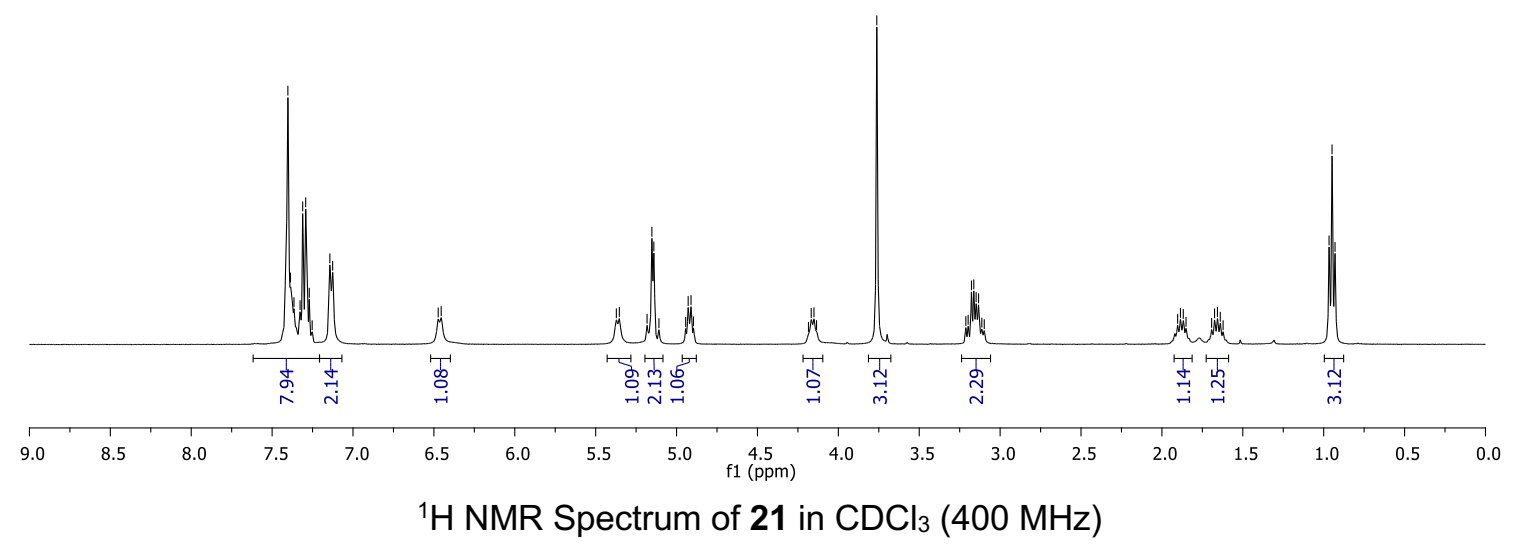




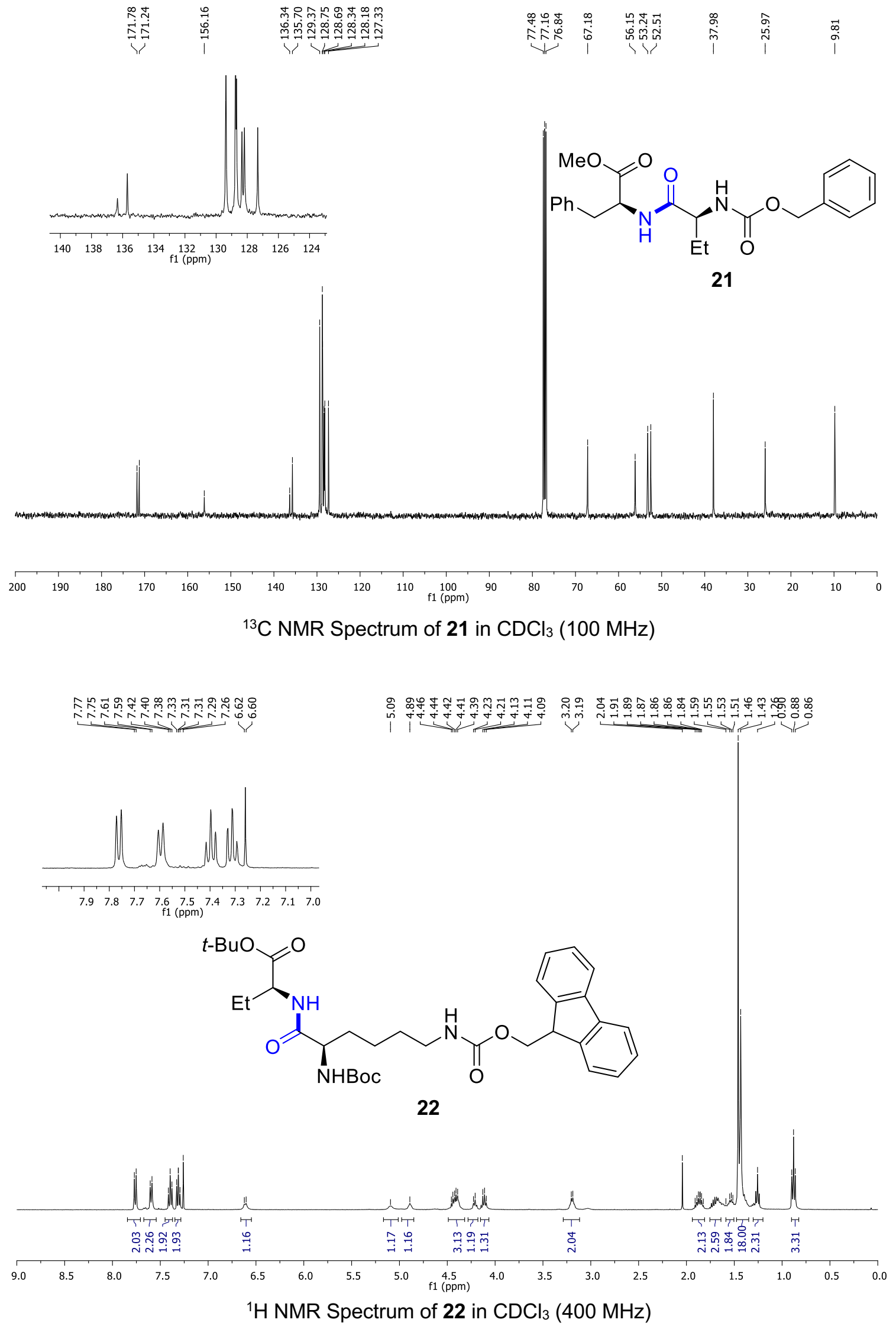



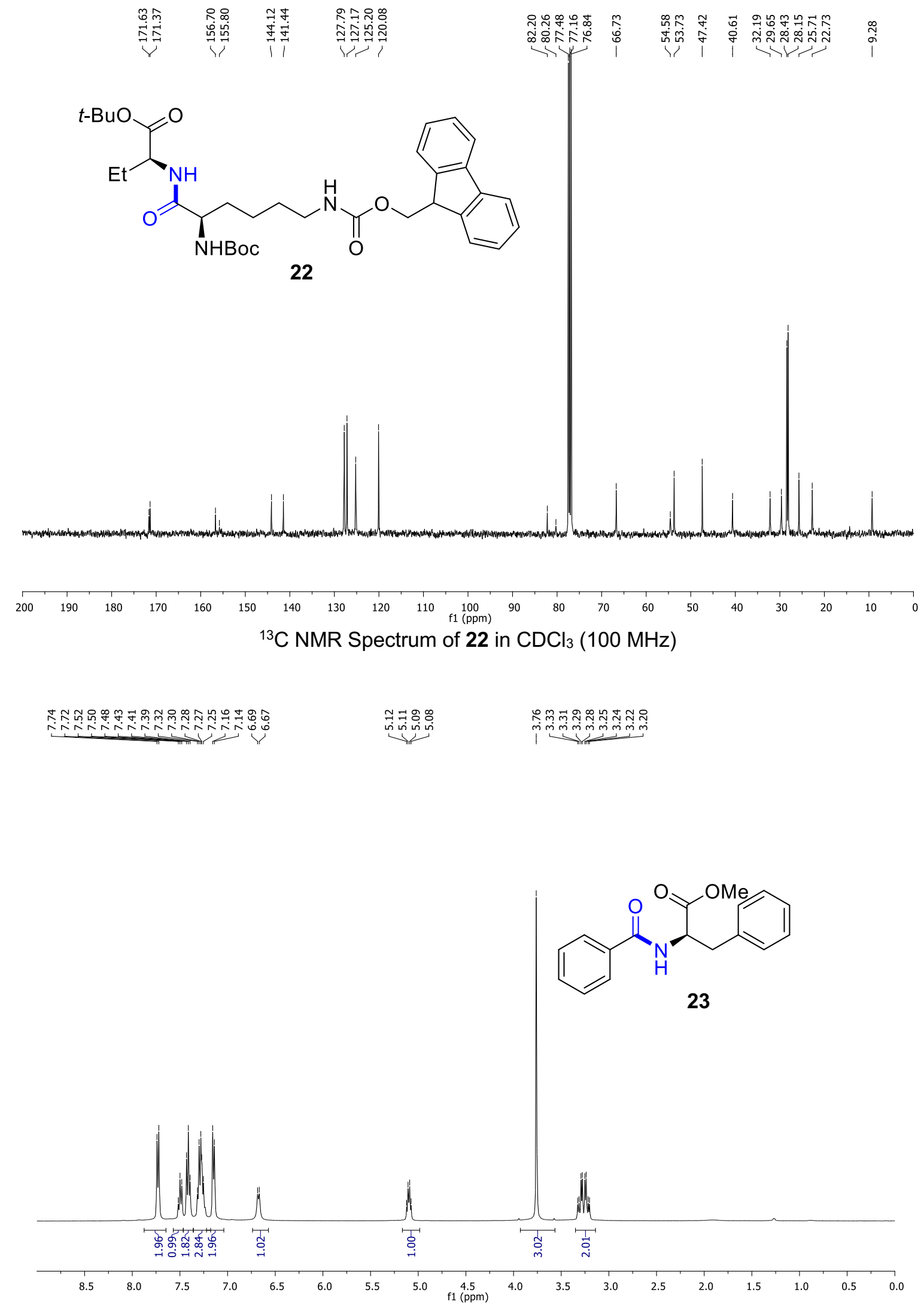

${ }^{1} \mathrm{H}$ NMR Spectrum of 23 in $\mathrm{CDCl}_{3}(400 \mathrm{MHz})$ 

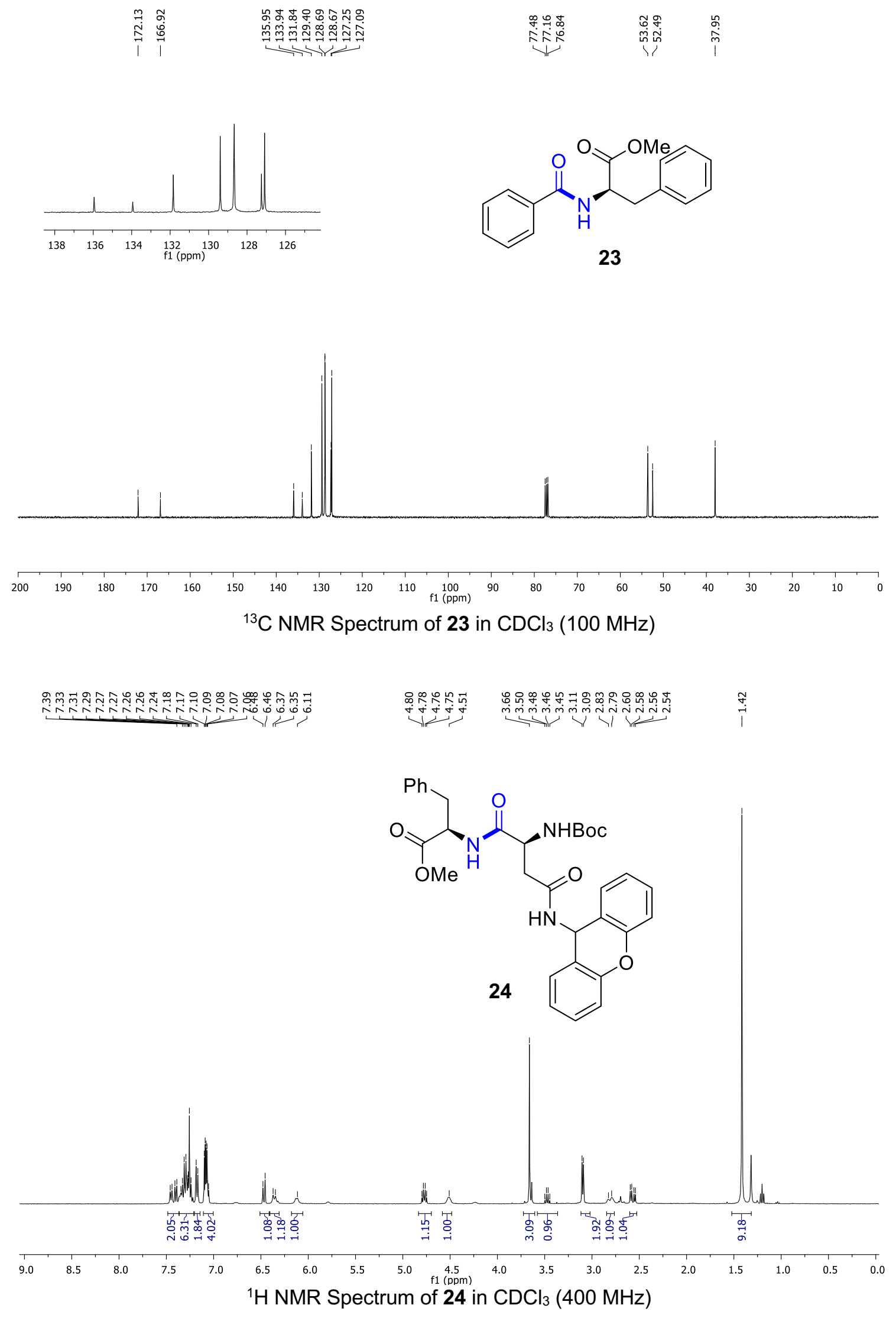


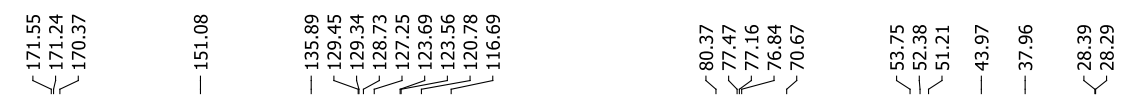<smiles>[Z4]c1cccc2c1C(NC(=O)C[C@H](N)C(=O)N[C@@H](Cc1ccccc1)C(=O)OC)c1ccccc1O2</smiles>
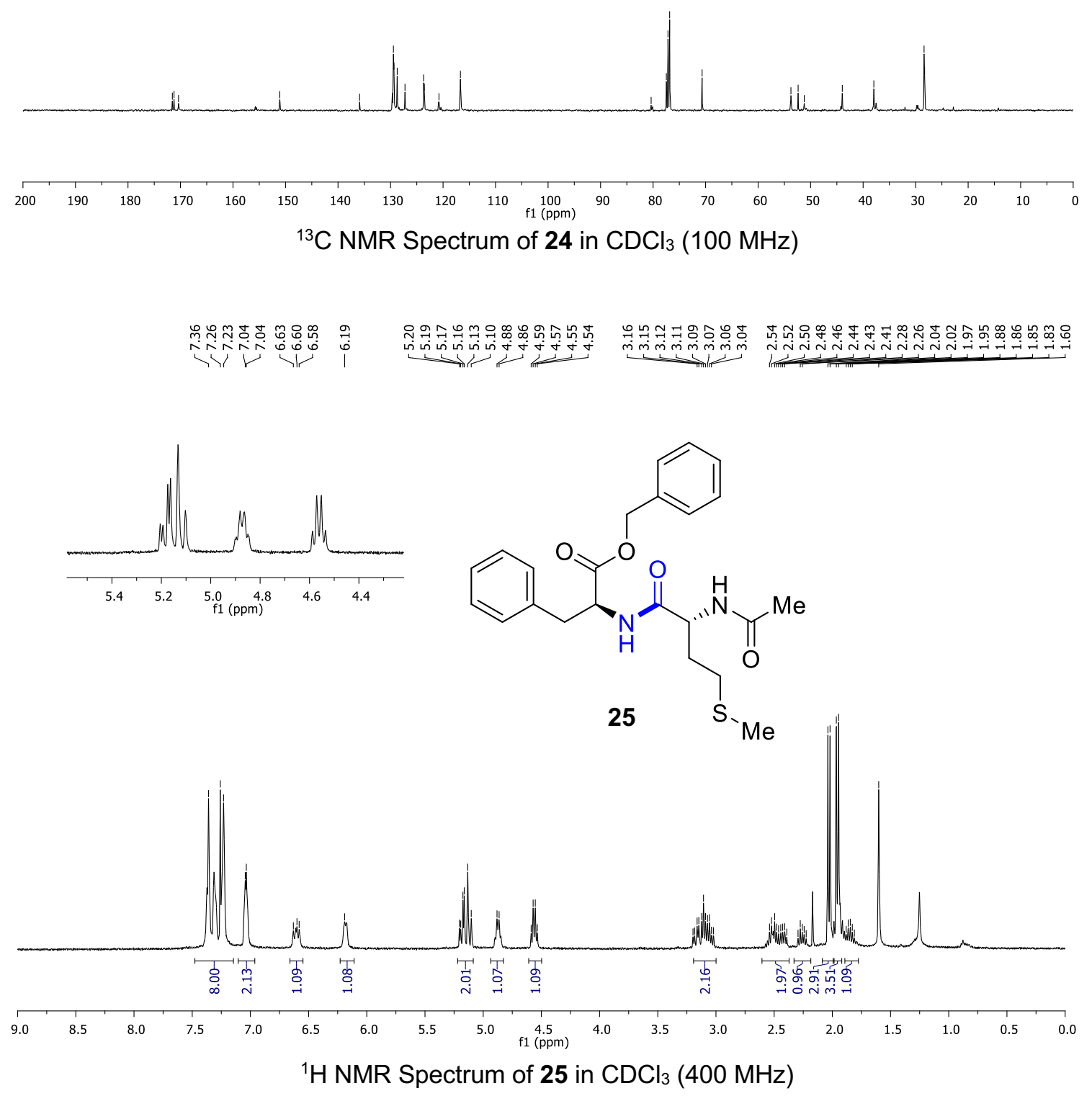

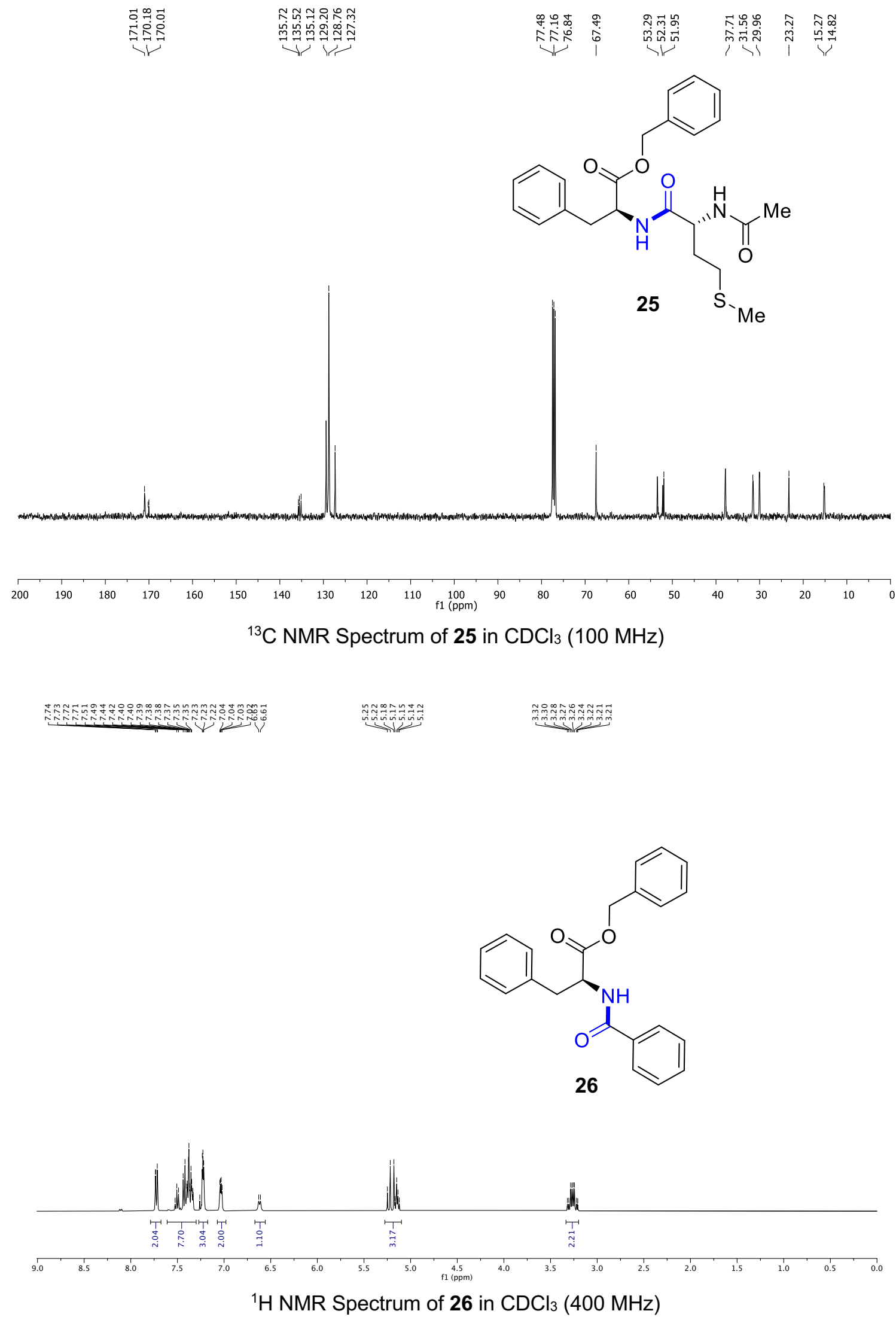


$$
\text { 覃 }
$$

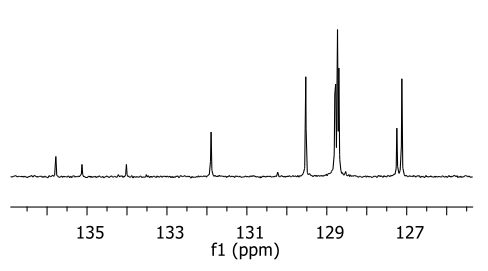

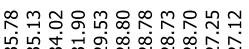

26
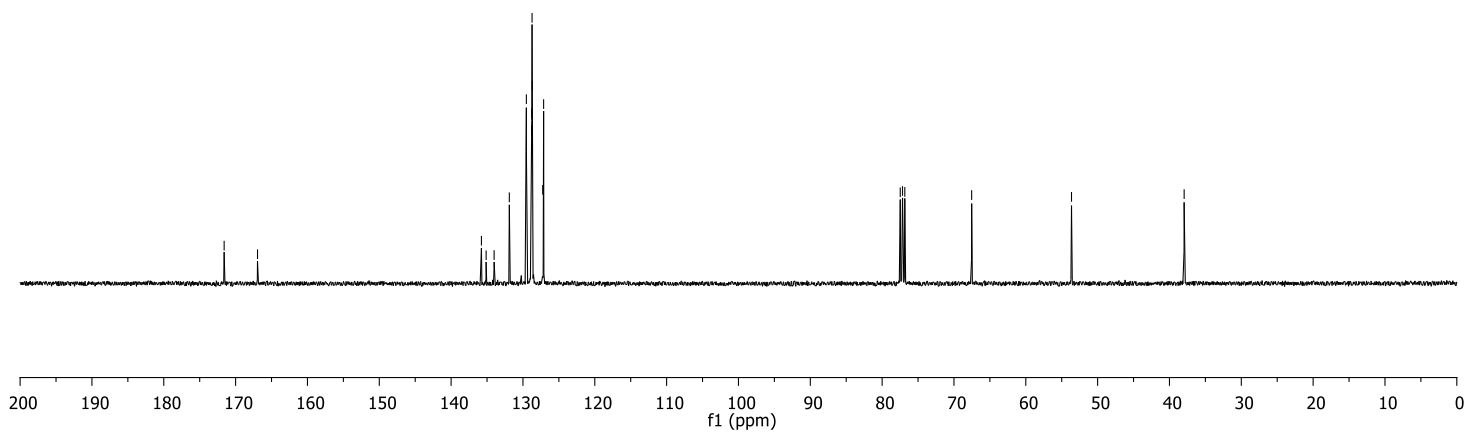

${ }^{13} \mathrm{C}$ NMR Spectrum of 26 in $\mathrm{CDCl}_{3}(100 \mathrm{MHz})$
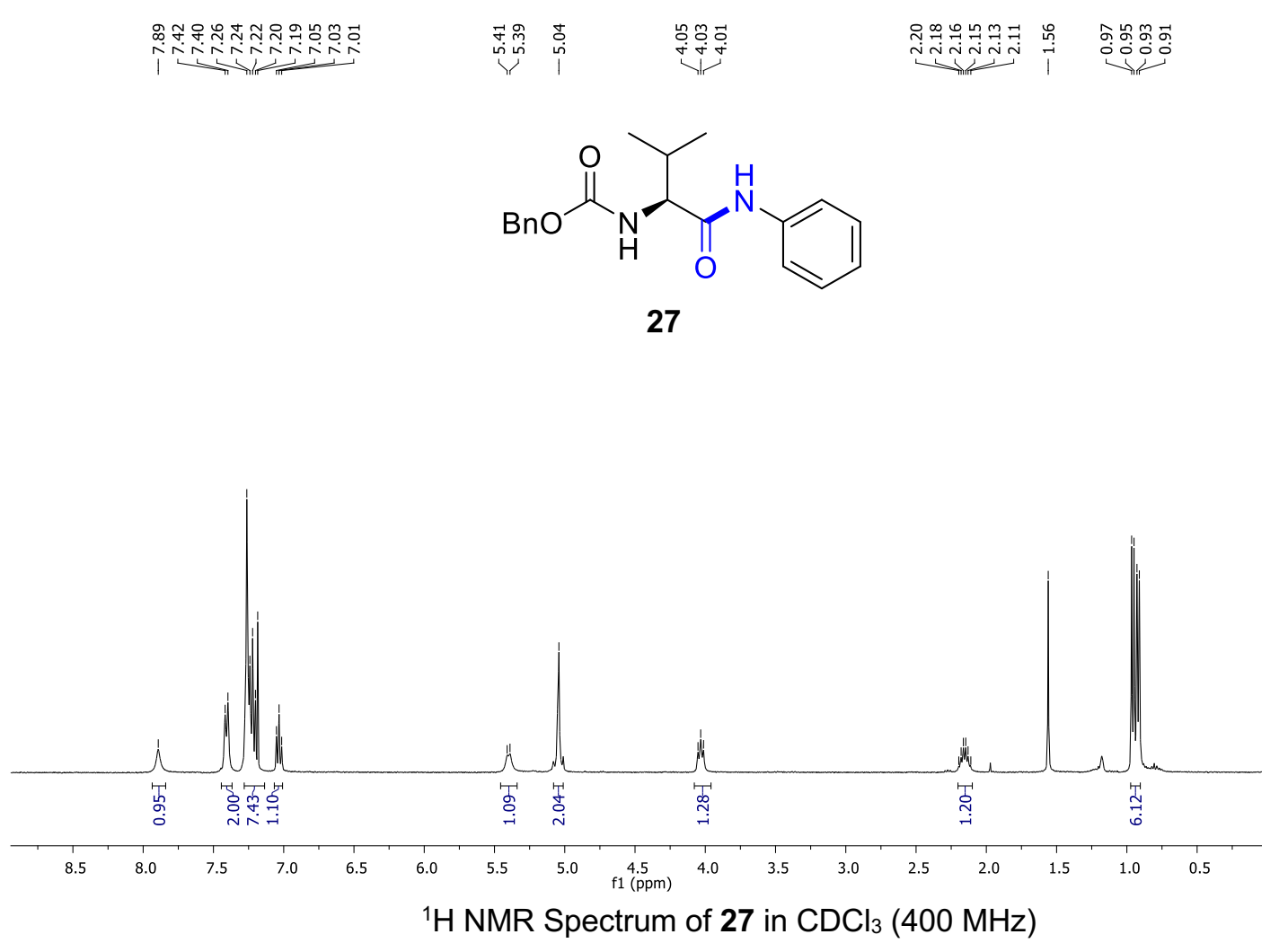


\section{HPLC DATA}

\section{Methyl benzoyl- $D L$-phenylalaninate standard ( $r a c-23)$}

Enantiomeric ratio was determined by HPLC with CHIRALCEL ${ }^{\circledR}$ OD-H column (hexane/i-PrOH $=90 / 10)$, flow rate $=1 \mathrm{~mL} / \mathrm{min}, 30{ }^{\circ} \mathrm{C}$<smiles>COC(=O)C(Cc1ccccc1)NC(=O)c1ccccc1</smiles>

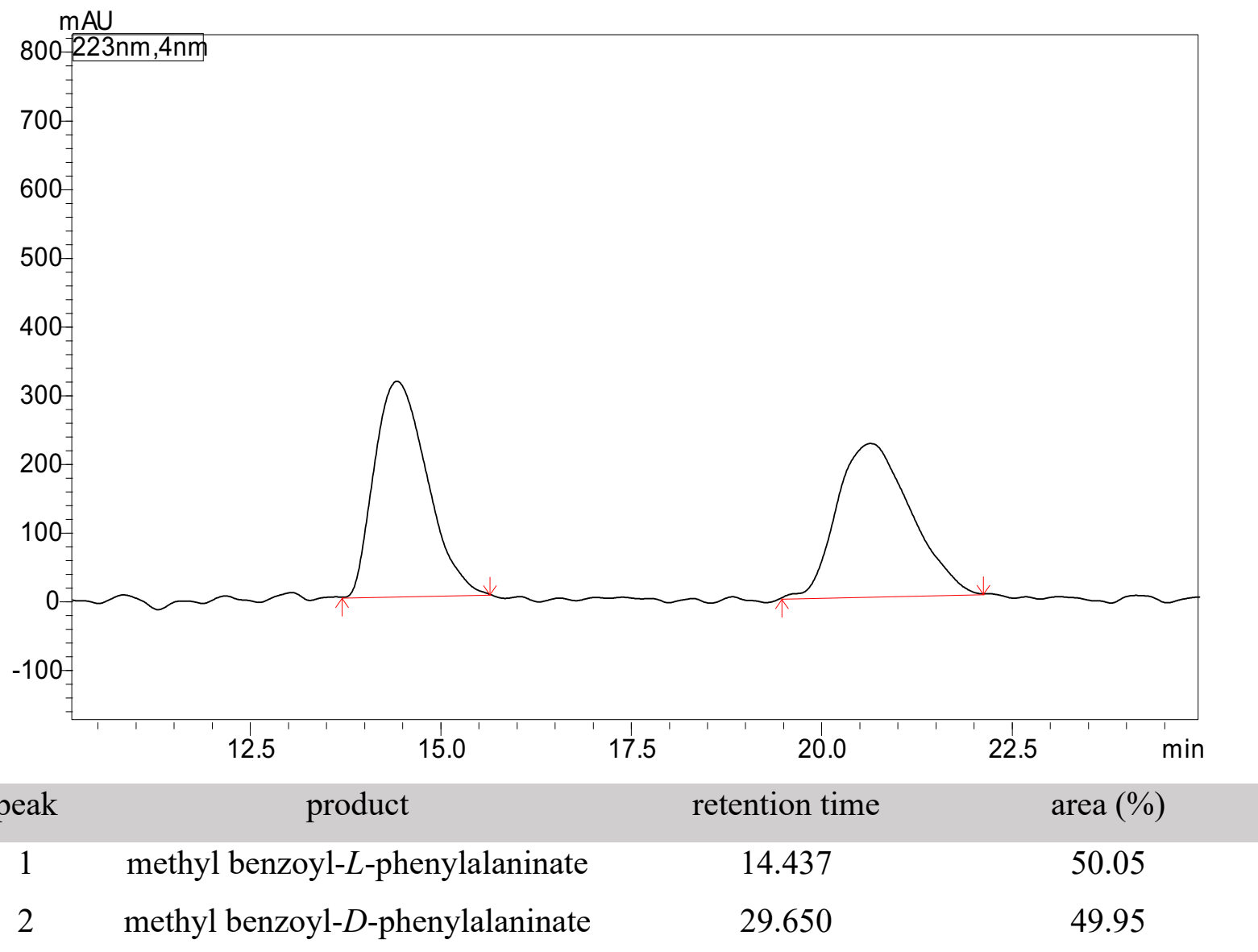


Methyl benzoyl- $L$-phenylalaninate (23)

Enantiomeric ratio was determined by HPLC with CHIRALCEL ${ }^{\circledR}$ OD-H column (hexane/i-PrOH $=90 / 10)$, flow rate $=1 \mathrm{~mL} / \mathrm{min}, 30{ }^{\circ} \mathrm{C}$

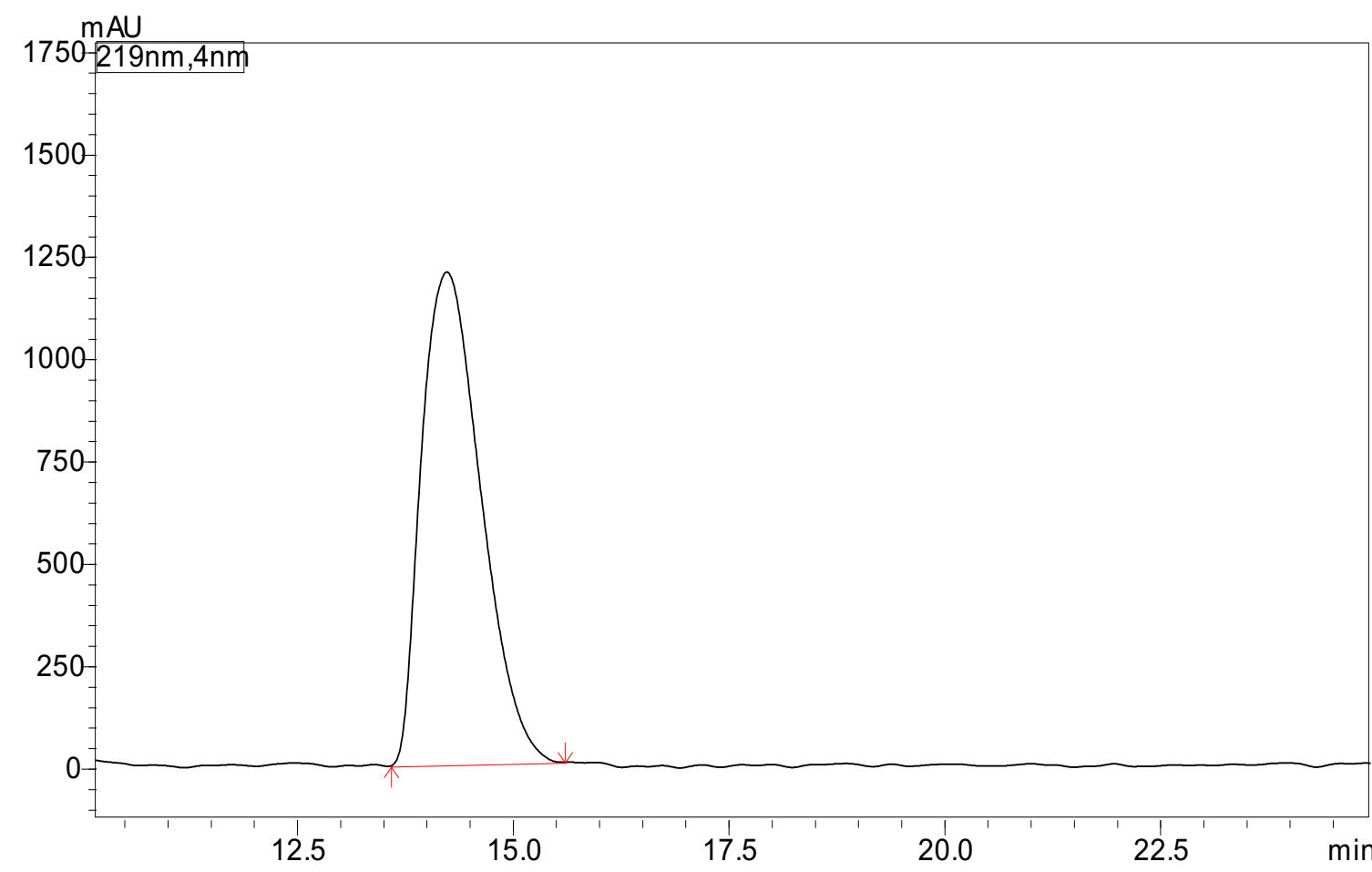

$\begin{array}{cccc}\text { peak } & \text { product } & \text { retention time } & \text { area }(\%) \\ 1 & \text { methyl benzoyl- } L \text {-phenylalaninate } & 14.250 & 100.0\end{array}$


Benzyl (D,L)-(3-methyl-1-oxo-1-(phenylamino)butan-2-yl)carbamate (rac-24) Enantiomeric ratio was determined by HPLC with CHIRALCEL ${ }^{\circledR}$ OJ-H column (hexane $/ i$-PrOH =90/10), flow rate $=1 \mathrm{~mL} / \mathrm{min}, 40{ }^{\circ} \mathrm{C}$

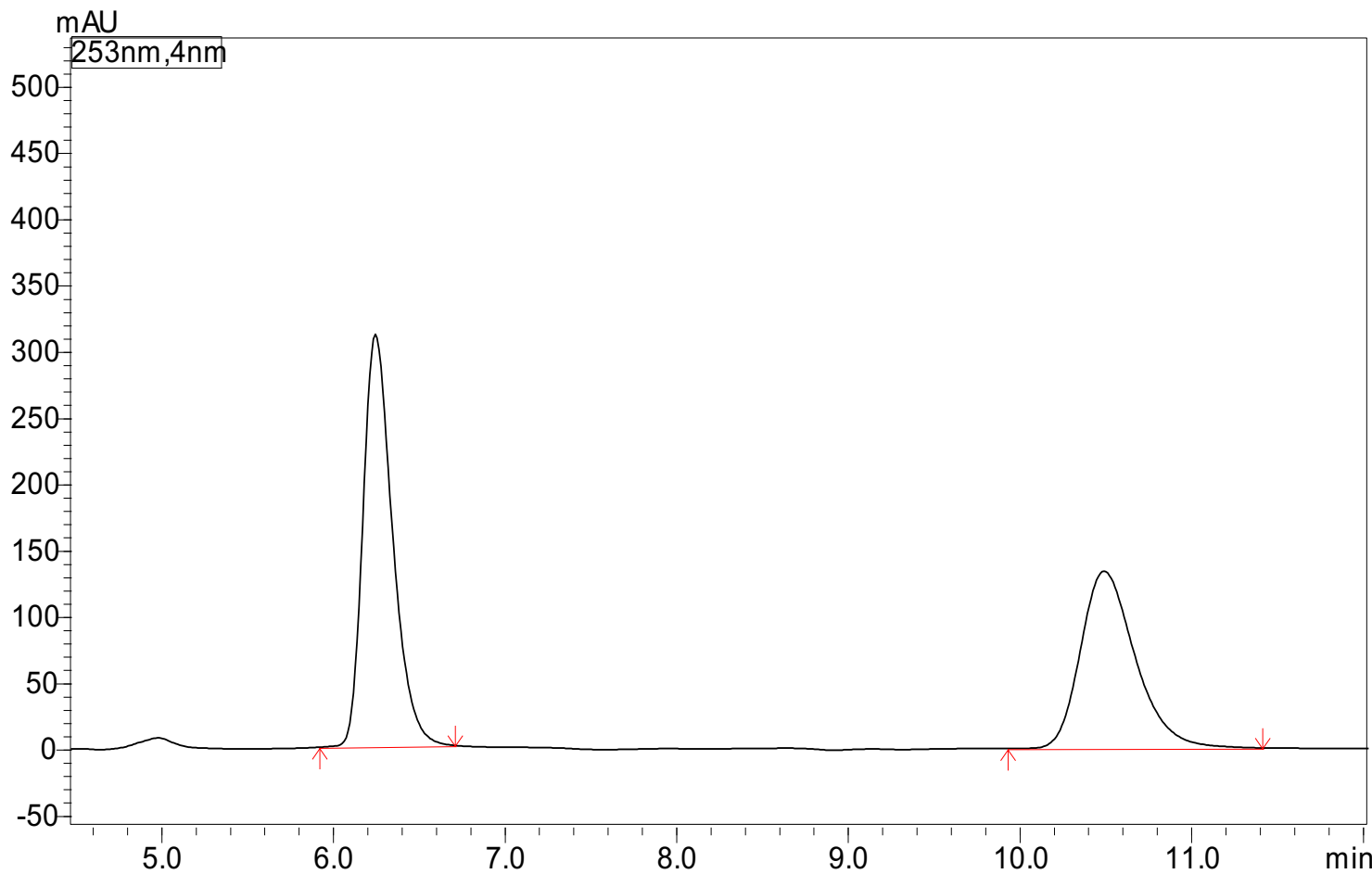

\begin{tabular}{clcr} 
peak & \multicolumn{1}{c}{ product } & retention time & area $(\%)$ \\
1 & $\begin{array}{l}\text { Benzyl }(S)-(3-\text { methyl-1-oxo-1- } \\
\text { (phenylamino)butan-2- }\end{array}$ & 6.251 & 49.31 \\
2 & $\begin{array}{l}\text { yl)carbamate } \\
\text { Benzyl }(R)-(3-m e t h y l-1-\text { oxo-1- } \\
\text { (phenylamino)butan-2- } \\
\text { yl)carbamate }\end{array}$ & 10.497 & 50.69
\end{tabular}




\section{Benzyl (S)-(3-methyl-1-oxo-1-(phenylamino)butan-2-yl)carbamate $(L-24)$}

Enantiomeric ratio was determined by HPLC with CHIRALCEL ${ }^{\circledR}$ OJ-H column (hexane/i-PrOH $=90 / 10)$, flow rate $=1 \mathrm{~mL} / \mathrm{min}, 40{ }^{\circ} \mathrm{C}$

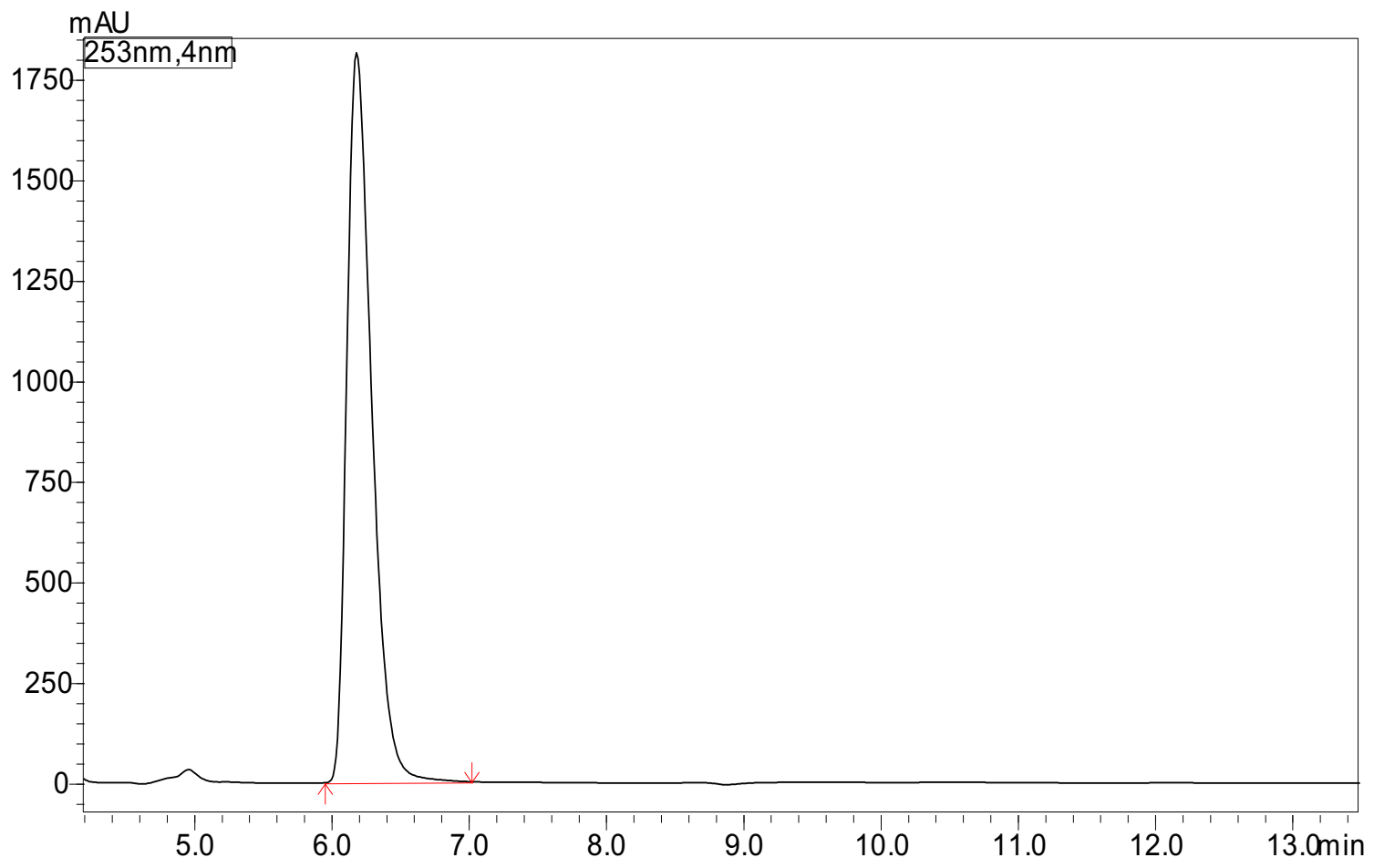

\begin{tabular}{clcc} 
peak & \multicolumn{1}{c}{ product } & retention time & area $(\%)$ \\
1 & $\begin{array}{l}\text { Benzyl }(S)-(3-\text {-methyl-1-oxo-1- } \\
\text { (phenylamino)butan-2- } \\
\text { yl)carbamate }\end{array}$ & 6.188 & 100 \\
& & &
\end{tabular}

